\title{
Higher-Dimensional Extensions of Nonlinear Inverse Reconstruction for Magnetic Resonance Imaging
}

\author{
Dissertation \\ for the award of the degree \\ "Doctor rerum naturalium" (Dr.rer.nat) \\ of the Georg-August-Universität Göttingen \\ within the doctoral program \\ "Physics of Biological and Complex Systems" \\ of the Georg-August University School of Science (GAUSS) \\ submitted by \\ Hans Christian Martin Holme \\ from Soest
}

Göttingen, 2019 


\section{Thesis COMMITTEE:}

Prof. Dr. Martin Uecker

Institut für Diagnostische und Interventionelle Radiologie

Universitätsmedizin Göttingen

Prof. Dr. Stefan Luther

Forschungsgruppe Biomedizinische Physik

Max-Planck-Institut für Dynamik und Selbstorganisation, Göttingen

PD Dr. Peter Dechent

Kognitive Neurologie

Universitätsmedizin Göttingen

\section{MEMBERS OF THE EXAMINATION BOARD:}

First REVIEWER:

Prof. Dr. Martin Uecker

SECOND REVIEWER:

Prof. Dr. Stefan Luther

Further MEMBERS OF THE EXAMINATION BOARD:

PD Dr. Peter Dechent

Prof. Dr. Russell Luke

Institut für Numerische und Angewandte Mathematik

Georg-August-Universität Göttingen

Prof. Dr. Marina Bennati

Forschungsgruppe EPR-Spektroskopie

Max-Planck-Institut für biophysikalische Chemie, Göttingen

Prof. Dr. Susann Boretius

Abteilung Funktionelle Bildgebung

Deutsches Primatenzentrum, Göttingen

Date of the oral examination: 19th September 2019 


\section{Contents}

1. Introduction 1

2. Magnetic Resonance and Image Reconstruction 5

2.1. Principles of Magnetic Resonance Imaging . . . . . . . . . . . . . 5

2.2. Acquisition Sequences . . . . . . . . . . . . . . 11

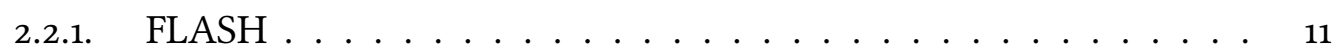

2.2.2. Balanced Steady-State Free Precession . . . . . . . . . . . . . 12

2.3. Image Reconstruction . . . . . . . . . . . . . . . . . . . . 13

2.3.1. Parallel MRI as an Inverse Problem . . . . . . . . . . . . . 13

$2.3 .2 . \quad$ NLINV . . . . . . . . . . . . . . . . . . . . . 14

2.3.3. Compressed Sensing . . . . . . . . . . . . . . . 16

3. MRI System and Hardware 17

4. Rank-relaxed MRI Signal Model for Robust Reconstruction 19

4.1. $\quad$ ENLIVE: An Efficient Nonlinear Method for Calibrationless and Robust

Parallel Imaging . . . . . . . . . . . . . . . . . . . . . 19

4.1.1. Introduction . . . . . . . . . . . . . 21

4.1.2. Theory . . . . . . . . . . . . . . 22

$4.1 .3 . \quad$ Results . . . . . . . . . . . . . . . . 25

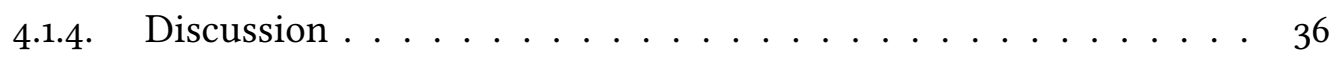

$4.1 .5 . \quad$ Conclusion $\ldots \ldots \ldots \ldots \ldots$

4.1.6. Methods . . . . . . . . . . . . . . . . . . 39

$4.1 .7 . \quad$ Appendix . . . . . . . . . . . . . . . . . 42

4.2. Pre-Calibration of Coil Sensitivities using ENLIVE . . . . . . . . . . . 44

4.3. $\quad$ ENLIVE with Temporal Regularization: Robust Reconstruction for realtime MRI . . . . . . . . . . . . . . . . . . . 48 
5. Nonlinear Reconstruction with Additional Spatial and Temporal Dimensions 53

5.1. Theory and Implementation $\ldots \ldots \ldots \ldots$. . . . . . . . . 55

5.2. Experiments . . . . . . . . . . . . . . 56

$5 \cdot 3 . \quad$ Results . . . . . . . . . . . . . . . . . . . 58

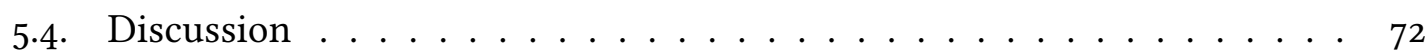

6. Banding-free Balanced Steady-State Free Precession 75

$6.1 . \quad$ Introduction . . . . . . . . . . . . . . . . 75

$6.2 . \quad$ VC-NLINV . . . . . . . . . . . . . . . . . 76

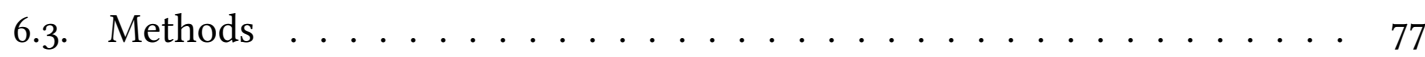

$6.4 . \quad$ Results . . . . . . . . . . . . . . . . . 77

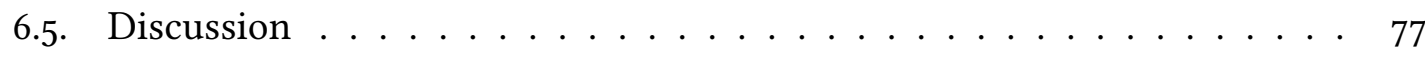

7. Summary and Outlook 83

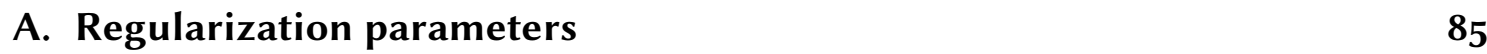

\begin{tabular}{|r|r|}
\hline Bibliography & 87
\end{tabular}

\begin{tabular}{|l|l}
\hline Danksagung & 97
\end{tabular}

\begin{tabular}{lr}
\hline Curriculum Vitæ & 98
\end{tabular} 


\section{Glossary}

ADMM .... alternating direction methods of multipliers

API . . . . . application programming interface

BART . . . . . Berkeley Advanced Reconstruction Toolbox

bSSFP . . . . . balanced steady-state free precession

CG . . . . . conjugate gradient method

CS ......... compressed sensing

CSF ........ cerebrospinal fluid

DCE .... . . dynamic contrast enhanced

ECG ..... electrocardiogram

ENLIVE . . . . . extended non-linear inversion based on ESPIRiT

FFT . . . . . . fast (discrete) Fourier transform

FID ..... free induction decay

FISTA . . . . . fast iterative shrinkage/thresholding algorithm

FLASH . . . . fast low-angle shot

fm-SSFP . . . . f frequency-modulated steady-state free precession

FOV . . . . . field of view

GRAPPA . . . generalized autocalibrating partially parallel acquisition

GRASP . . . . golden-angle radial sparse parallel MRI 
Glossary

IRGNM . . . . iteratively regularized Gauss-Newton method

MPRAGE . . . magnetization-prepared rapid gradient-echo

MRI .... . magnetic resonance imaging

NLINV . . . . regularized non-linear inverse reconstruction

NMR .... nuclear magnetic resonance

nuFFT . . . . non-uniform fast Fourier transform

PICS . . . . . combined parallel imaging and compressed sensing

rf . . . . . . radio frequency

SAKE ..... simultaneous autocalibrating and k-space estimation

SENSE ..... sensitivity encoding

SNR ...... signal-to-noise ratio

SSA-FARY . . . singular spectrum analysis for advanced reduction of dimensionalty

SVD ....... singular value decomposition

$T_{1} \ldots \ldots$. . spin-lattice relaxation time

$T_{2} \quad \ldots \ldots$ spin-spin relaxation time

$T_{2}^{*} \ldots \ldots$ effective spin-spin relaxation time

TV ....... total variation

XD-GRASP . . . extra-dimensional GRASP 


\section{Introduction}

Magnetic resonance imaging (MRI) is one of the techniques of choice in clinical radiology. Since its introduction in the 1980s, its use has been steadily expanded and it is now routinely prescribed for orthopedic, neurological, gastrointestinal, and cardiac indications. However, as the number of MRI scanners is limited, waiting times for MRI exams have remained long. [1]

A large speedup has been achieved in the 1980 os by the introduction of faster imaging sequences, which make use of increased gradient performance, first and foremost with the introduction of fast low-angle shot (FLASH) [2-4

Since then, MRI has steadily gotten faster and more detailed: the increase in field strength from $1.5 \mathrm{~T}$ to $3 \mathrm{~T}$ in clinical practice has provided increased signal-to-noise ratio (SNR), while the better gradient performance in newer scanners has done its share to reduce acquisition times. Today, the new limit on acquisition speed is no longer the hardware, but rather the time required for sufficient sampling and physiological limits on gradient-induced peripheral nerve stimulation and heating of the human body by the radio frequency (rf) excitation pulses.

The next major innovation came with parallel imaging [5-7]: by using multiple receive coils and leveraging their inherent spatial redundancy, sampling below the Nyquist limit (commonly referred to as undersampling) is possible. While FLASH reduced acquisition times form minutes to seconds [4], parallel imaging allows reconstruction of images from data acquired at fractions of a second [8]. However, parallel imaging needs accurate calibration of the coil sensitivity profiles. Classically, this is done as a pre-processing step followed by the image reconstruction, which can then be described as a linear inverse problem for the image. However, this is suboptimal, as it does not make full use of the available data. Instead, the non-linear problem in which both coil sensitivities and image are regarded as unknowns can also be solved directly. In particular, regularized nonlinear inverse reconstruction (NLINV) [9] estimates image and coil sensitivity profiles simultaneously, thereby making optimal use of the available data.

A further innovation came in the form of compressed sensing (CS) From lossy signal compression (such as JPEG and mp3), it is known that real signals are compressible. 


\section{Introduction}

That means that they contain fewer degrees of freedom than, for example, their pixel count would indicate. More precisely, there is some transformation (such as the discrete cosine transform used in JPEG and mp3) under which the signal representation is sparse, meaning that this transformed representation only contains few important coefficients. As Block, Uecker, and Frahm [10] and Lustig, Donoho, and Pauly [11] showed, this can also be exploited in MRI, by using an incoherent acquisition and constraining images to be sparse under a certain transformation. Compressed sensing can naturally be combined with parallel imaging, allowing reconstruction form even fewer data.

These approaches all improve on the reconstruction of images in various settings. Separately, there has been a continuing development of model-based reconstruction methods [12 14]. Instead of the comparatively simple signal model used in conventional MRI image reconstruction, more of the physical processes in MRI are modeled such as relaxation constants or magnetic field properties like inhomogeneity and off-resonance. On one hand, this enables efficient reconstruction of the corresponding parameter maps, but on the other hand it leads to a stricter signal model which is more easily violated, if the actual measurement does not match the assumptions. Since violations of the signal model, both in imaging and in model-based methods, cause artifacts and unusable results, avoiding them is paramount.

In this thesis, I will investigate how multi-dimensional extensions to regularized nonlinear inverse reconstruction (NLINV) can be used to increase reliability, flexibility and, robustness in image reconstruction. In contrast to model-based approaches, this is done without using specific assumptions about the underlying physical laws. This idea will be explored in different experimental settings such as imaging with phase constraints, phase singularities, non-Cartesian acquisitions and real-time MRI and cardiac self-gating.

In Chapter 2 I will provide a short introduction into the physical background of MRI and into the mathematics needed for MRI reconstruction. Chapter 3 gives basic information about the experimental setup and the used software environment.

Based on the core idea of adding additional degrees of freedom to make image reconstruction more robust, Chapter 4 introduces extended non-linear inversion based on ESPIRiT (ENLIVE) a novel method for parallel imaging which relaxes the MRI model by allowing multiple images and set of coil sensitivity profiles, increasing the dimensionality of the problem. It integrates ideas from ESPIRiT [15], into NLINV] substantially improving upon both of these methods. ENLIVE provides robustness against an insufficient field

${ }^{1}$ In principle, all $\mid$ MRI $\mid$ methods are necessarily based on some signal model. The term model-based reconstruction, however, has traditionally been reserved for methods which employ models more complicated than the simple Fourier-based signal model. 
of view (FOV) in parallel imaging, phase singularities and aliasing in phase-constraint imaging. As this method is of wider interest, I published it in "Scientific Reports" and the corresponding manuscript is reprinted as Section 4.1

In Chapter 5 another way of extending image reconstruction is explored. This approach first extends NLINV to arbitrary dimensions, such as time, space shifts and cardiac and respiratory state. Correlations in the multi-dimensional data are then exploited using variational regularization terms, without assuming a specific model. In particular, this includes the the extension of NLINV to compressed sensing

Chapter 6 introduces a method for banding-free reconstruction in balanced steady-state free precession (bSSFP), by shifting the phase increment of the rf-pulse and including these phase cycles as additional coils in the reconstruction.

Finally, Chapter 7 summarizes the findings of each of the preceding chapter and includes an outlook towards future work. 



\section{Magnetic Resonance and Image Reconstruction}

\subsection{Principles of Magnetic Resonance Imaging}

This section is based on Chapter 2 of Holme [16], which follows Chapters 1 and 9 of Haacke et al. [17]. A general introduction into nuclear magnetic resonance (NMR) and magnetic resonance imaging (MRI) can be found for example in Bernstein, King, and Zhou [18] or Haacke et al. [17].

Magnetic resonance imaging (MRI) is fundamentally based on the interaction of the nuclear magnetic moment, generated by the nuclear spin, with an external magnetic field $\boldsymbol{B}_{0}$. While different nuclei can be used in MRI the most common one is the hydrogen nucleus found in water, fat and other molecules.

When such a hydrogen nucleus is placed in an external magnetic field $\boldsymbol{B}_{0}$, there is an energy difference of $\hbar \gamma B_{0}$ between the parallel and anti-parallel alignment of the spin because of the Zeeman effect, where $\hbar$ is the reduced Planck constant and $\gamma$ is the nucleus-dependent gyromagnetic ratio.

With this, the spin, and thereby the magnetic moment of the nucleus, is constrained to two values in the direction parallel to the external magnetic field. There is however, no constraint on the magnetization orthogonal to the magnetic field, which therefore proceeds to precess around the direction of the external magnetic field, analogously to how a classical magnetic moment would behave. Their precession frequency is the Larmor frequency $\omega_{0}=\gamma B_{0}$. However, since there is no imposed coherence on the precession of different nuclei, they will not superimpose constructively in the transversal plane and cancel out in a sample made up of a multitude of atoms. But as a consequence of the aforementioned energy difference between parallel and anti-parallel alignment, there will be a bias towards alignment parallel to the external magnetic field, as that is the state of lower energy. At body temperature, this energy difference is small compared to thermal energy, leading to a spin excess of approximately $N \hbar \omega_{0} / 2 k_{b} T$ [19. pg. 4], where $k_{b}$ is the 
Boltzmann constant, $T$ the temperature and $N$ the total number of spins in the sample. For an external field of $3 \mathrm{~T}$, for example, the spin excess at body temperature is about 20 per million. While this longitudinal magnetization is small, it is still large enough for MRI

Using a second, time-varying magnetic field, it is possible to create a macroscopic transversal magnetization. Resonant excitation of the nuclear spins is necessary for this, that means that the frequency of the second magnetic field $B_{1}(t)$ is the Larmor frequency. While originally oriented parallel to $\boldsymbol{B}_{0}$, such an excitation pulse $\boldsymbol{B}_{1}(t)$ tilts this magnetization towards the transversal plane. As the Larmor frequency is in the radio frequency range for commonly used field strengths and nuclei, such pulses are called radio frequency (rf) pulses. The angle by which the equilibrium magnetization is deflected is proportional to the time integral of the envelope of $\boldsymbol{B}_{1}(t)$ and a pulse with a deflection of $\alpha$ is called an $\alpha$-pulse. Microscopically, the action of an $\mathrm{rf}$ pulse can be viewed as a combination of two effects: (1) it imposes phase coherence on the precessing spins, thereby creating a macroscopic transversal magnetization and (2) it excites spins from the lower energy parallel state into the higher energy anti-parallel state, thereby reducing the longitudinal magnetization. The precession of the magnetization after an excitation pulse induces a time-varying magnetic flux in nearby coils, which can be detected.

After excitation, however, the system will return into its equilibrium state, where the bulk magnetization is parallel to the external field.

In longitudinal direction, spins excited into the higher-energy anti-parallel state will flip back into the parallel state, thereby leading to a recovery of the longitudinal magnetization according to:

$$
M_{\|}(t)=M_{0}\left(1-\mathrm{e}^{-\frac{t}{T_{1}}}\right)
$$

with the equilibrium magnetization $M_{0}$ and the spin-lattice relaxation time $\left(T_{1}\right)$

In the transversal plane, individual spins are also affected by the magnetic fields of their neighbors, leading to slightly different Larmor frequencies for each spin. This in turn leads to a loss of coherence and thereby to a loss of transversal magnetization according to:

$$
M_{\perp}(t)=M_{0} \mathrm{e}^{-\frac{t}{T_{2}}}
$$

where $T_{2}$ is the spin-spin relaxation time $\left(T_{2}\right)$.

Typical values of $T_{2}$ are between $1 \mathrm{~ms}$ to $100 \mathrm{~ms}$; typical values of $T_{1}$ are between $100 \mathrm{~ms}$ to $1000 \mathrm{~ms}$.

The excitation process and both $T_{1}$ and $T_{2}$ relaxation can be described concisely using 
the Bloch equation [20], a differential equation for the macroscopic magnetization $\boldsymbol{M}$ :

$$
\frac{\mathrm{d} \boldsymbol{M}}{\mathrm{d} t}=\boldsymbol{M} \times \gamma \boldsymbol{B}+\left(\begin{array}{c}
-\frac{M_{x}}{T_{2}} \\
-\frac{M_{y}}{T_{2}} \\
\frac{M_{0}-M_{z}}{T_{1}}
\end{array}\right)
$$

with $\boldsymbol{B}=\boldsymbol{B}_{0}+\boldsymbol{B}_{1}(t)$.

Applying an excitation pulse to a sample leads to a signal decaying with $T_{2}$ called free induction decay (FID) For imaging, it is often advantageous to create signals at later points, which are called echoes. The two most important types are spin echoes and gradient echoes.

Spin Echo After an initial excitation pulse, the spins begin to dephase due to $T_{2}$ decay. By then applying a $180^{\circ}$-pulse at a time $\tau$ after the excitation pulse, the spins are flipped into the transversal plane. This means that spins which accumulated excess phase because of a locally higher Larmor frequency now lag behind, while spins which precess slower are now leading. At a time $T=2 \tau$, these spins are exactly in phase again, creating an echo. The time between initial excitation pulse and the echo is called TE, the echo time. The echo is still subject to $T_{2}$ decay, so it is attenuated by a factor $\mathrm{e}^{-T E / T_{2}}$. A diagram of a spin echo is shown in Figure 2.1(a)

Gradient Echo Here, a magnetic gradient field is applied after the excitation pulse, dephasing the spins. By then applying a gradient of opposite polarity, they can be rephased again, creating an echo when the integrated area under the gradients is zero. Here, in contrast to the spin echo, magnetic field inhomogeneities do not cancel out. Therefore, the echo is attenuated by the shorter effective spin-spin relaxation time $\left(T_{2}^{*}\right)$ according to $\mathrm{e}^{-T E / T_{2}^{*}}$, instead of $T_{2}$. A diagram depicting a gradient echo is shown in Figure 2.1(b)

To generate an image from these signals it is necessary to relate the signal to spatial position. This can be done by exploiting the linear dependence of the Larmor frequency on the magnetic field strength: by applying a gradient field $G(t)$ varying linearly in, for example, the $x$-direction, the total magnetic field becomes $B(x)=B_{0}+x G(t)$. This changes the Larmor frequency to $\omega(x)=\omega_{0}+\gamma x G(t)$. Since the Larmor frequency now depends on the spatial position, this is called frequency encoding. The recorded signal $s(t)$ in MRI depends on the spin density $\rho(\boldsymbol{r})$ and on the phase $\phi(\boldsymbol{x}, t)=-\int_{0}^{t} \omega(\boldsymbol{r}, t) \mathrm{d} t$ of the spins [17 pg. 141]:

$$
s(t)=\int \rho(\boldsymbol{r}) \mathrm{e}^{\mathrm{i}\left(\omega_{0} t+\phi(\boldsymbol{r}, t)\right)} \mathrm{d}^{3} r .
$$




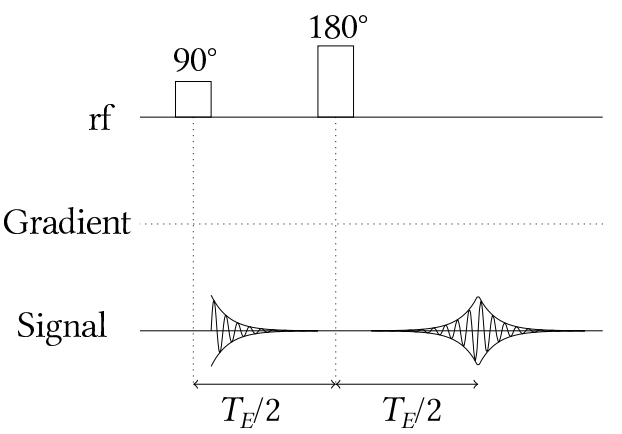

(a)

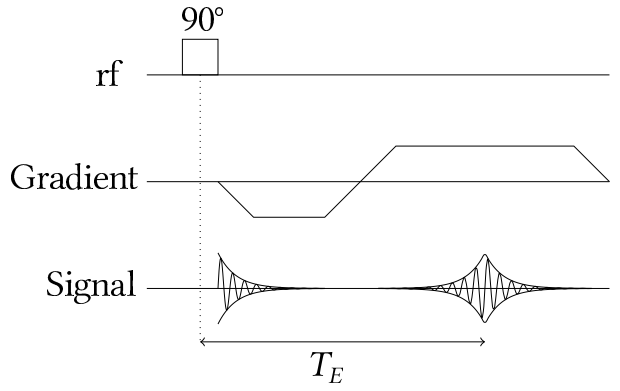

(b)

Figure 2.1: Diagram depicting a (a) spin echo and (b) a gradient echo. For the spin echo in (a) no gradients are needed: the singal decays after an initial $90^{\circ}$. By applying a $180^{\circ}$-pulse after $T_{E} / 2$, the spin rephase at $T_{E}$, creating an echo. For the gradient echo in (b) the spins are dephased with a gradient after the initial $90^{\circ}$-pulse. They are subsequently rephased with a gradient of opposite polarity, leading to an echo when the total area under the gradients is zero. Diagrams adapted from [21].

For a one dimensional object in $x$-direction, the signal after frequency encoding and demodulation of $\omega_{0}$ reduces to:

$$
s(t)=\int \rho(x) \mathrm{e}^{-\mathrm{i} \int_{0}^{t} \gamma x G(t) \mathrm{d} t} \mathrm{~d} x .
$$

Introducing $2 \pi k(t):=\gamma \int_{0}^{t} G(t) \mathrm{d} t$ leads to:

$$
s(k)=\int \rho(x) \mathrm{e}^{-2 \pi \mathrm{i} k x} \mathrm{~d} x
$$

where the time dependence is now implicit in $k . s(k)$ is exactly the Fourier transform of $\rho(x)$, and so the spin density can be obtained from a frequency-encoded measurement as the inverse Fourier transform of $s(k)$ :

$$
\rho(x)=\int s(k) \mathrm{e}^{2 \pi \mathrm{i} k x} \mathrm{~d} k
$$

This $k$ can be identified as a vector in the space of spatial frequencies, which is called $\mathrm{k}$-space in MRI $k$ can be determined as the integral of the applied gradient; this timeevolution of $k$ is referred to as the k-space trajectory.

The gradient echo sequence shown in Figure 2.1(b) is already a sequence which includes frequency encoding: during echo formation, a gradient constant in time is present. This gradient will encode spatial positions in the frequencies of the resulting signal. The gradient direction during signal acquisition is called the readout direction. In this view of the gradient echo sequence, the echo is identified with the central k-space point.

For real three-dimensional objects, the k-space formalism can be used to collect data 
along a three-dimensional k-space trajectory, thereby sampling the three-dimensional spin density

$$
s(\boldsymbol{k})=\int \rho(\boldsymbol{r}) \mathrm{e}^{-2 \pi \mathrm{i} k \boldsymbol{r}} \mathrm{d}^{3} r .
$$

In practice, it is not possible to sample the signal continuously but only at discrete time points.

Alternatively, the dimensionality of the problem can be reduced by only exciting a slice of the object to begin with. This is possible through the same idea: The Larmor frequency depends linearly on the magnetic field. Since excitation needs to be resonant, a gradient orthogonal to the desired slice is turned on during excitation. By tuning the frequency of the excitation to the Larmor frequency for the desired slice, only that slice is excited. In real settings, an excitation pulse will always have a finite frequency content, leading to excited slices of a certain thickness.

Two common $2 \mathrm{D}$ trajectories including slice selection, Cartesian and radial sampling, are shown in Figure 2.2. While arbitrarily long trajectories are possible in theory, real trajectories are limited by $T_{2}^{*}$ decay and off-resonance effects caused by imperfect magnetic fields. Therefore, real trajectories tend to use new magnetization for, for example, each line in k-space, as shown in Figure 2.2. For fast imaging trajectories, residual magnetization after signal acquisition is often purposefully destroyed (spoiled) or rephased, leading to different acquisition techniques discussed in Section 2.2. However, using new magnetization for each $\mathrm{k}$-space line necessitates acquisition of a large number excitations, which is why MRI is generally a comparatively slow technique.

In Cartesian imaging, gradients between excitation and read-out are used to shift the acquired slice in $\mathrm{k}$-space in a direction orthogonal to the readout direction. This is called phase encoding. By acquiring lines in k-space with the same separation as the separation between samples in readout direction, the acquired points lie on a Cartesian grid, which gives the sequence its name. Instead of slice selection, phase encoding can also be extended into the third dimension.

In radial encoding instead, a number of lines passing through the center of $\mathrm{k}$-space (called spokes, because of the analogy to spokes on a wheel) are acquired. This is advantageous for fast imaging, as the center of k-space is sampled in each acquisition. Furthermore, each spoke contains image information of equal importance. This is in contrast to Cartesian imaging, where, for example, the important center of k-space is only sampled once. This is an advantage when only few radial spokes are acquired, as in undersampled MRI, which will be explained in the last part of this section. An in-depth discussion is found, for example, in Block [22]. 

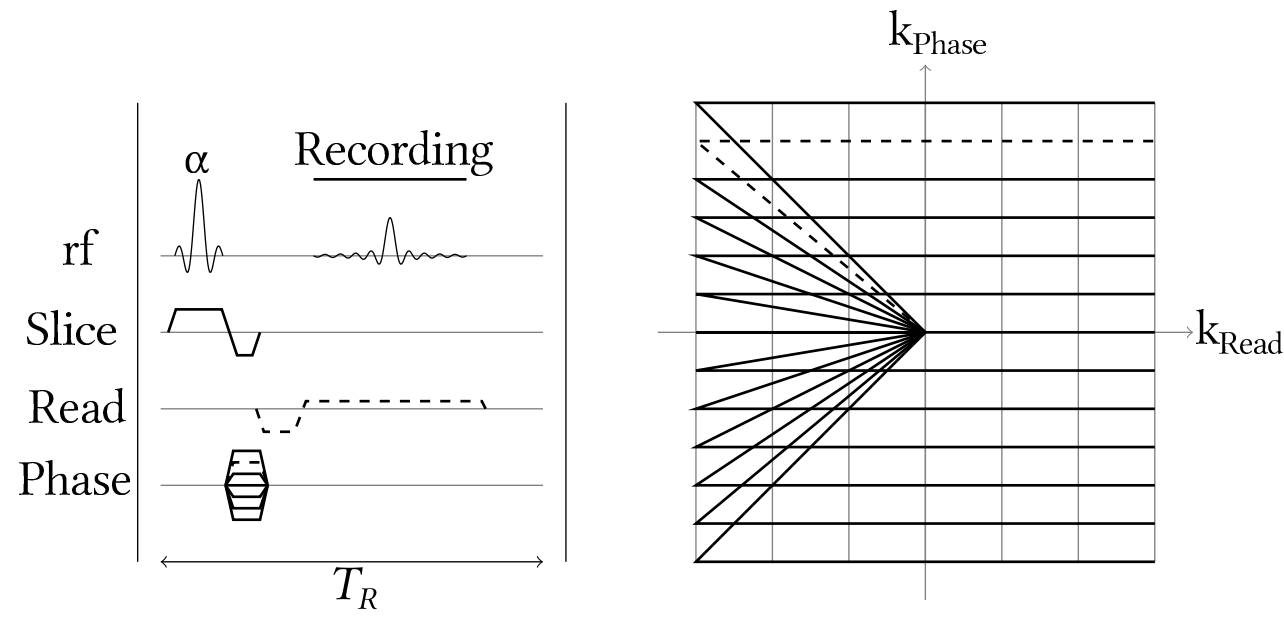

(a)
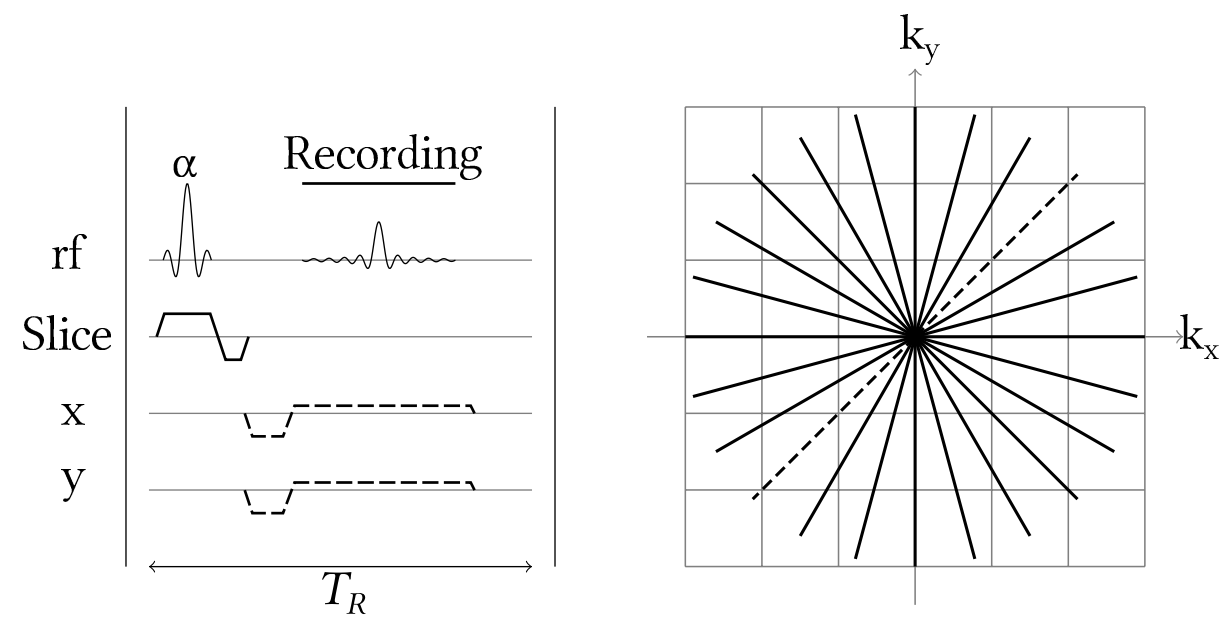

(b)

Figure 2.2: Sequence diagram for a single repetition (TR) and the corresponding $\mathrm{k}$-space diagrams for (a) Cartesian and (b) radial sampling. The dashed gradient line in the sequence diagram corresponds to the dashed $\mathrm{k}$-space line in the k-space diagram. Both contain an initial $\alpha$-pulse for excitation and a slice selection gradient. In (a) Cartesian encoding, only the phase-encoding gradient changes from TR to TR, the frequency-encoding gradient stays the same. In (b) radial encoding, the readout gradient is effectively rotated, it therefore has both components in both $x$ in $y$ in general. Diagrams adapted from [21]. 
In modern MRI imaging, multiple receive coils are used acquire the signal from an object. This is advantageous because it increases signal-to-noise ratio (SNR), as smaller coils can be placed closer to the object and multiple coils can, together, cover the same area as one larger coil. However, image reconstruction becomes more involved, as each coil only contains information about the part of the image where it is sensitive. This changes the signal equation to

$$
s_{j}(\boldsymbol{k})=\int c_{j}(\boldsymbol{r}) \rho(\boldsymbol{r}) \mathrm{e}^{-2 \pi \mathrm{i} k \boldsymbol{r}} \mathrm{d}^{3} r .
$$

$s_{j}$ is the signal detected by the $j^{\text {th }}$ coil and $c_{j}$ is the spatial sensitivity profile (also called coil profile or coil sensitivity profile) of that coil. To calculate a single image from the individual coil images, their root-sum-of-squares is normally calculated [23]. The coil sensitivity profiles are normally not known beforehand, and can change from measurement to measurement, since interactions with the imaged object can change the coil sensitivity profiles. They are therefore often estimated form specifically acquired pre-scans or from part of the acquired data in a step before reconstruction, called coil sensitivity calibration.

When using multiple coils, part of the spatial information is contained in the spatial location of the coils. This information can be used to replace time consuming sampling of k-space in a technique called parallel imaging, discussed in more detail in Section 2.3.1 In Cartesian imaging, this speedup comes from simply skipping phase-encoding lines; in radial imaging viewer radial spokes are acquired. Sampling of k-space below the Nyquist limit is referred to as undersampling and is responsible for a large part of the speedup MRI has seen in the last 30 years.

Reconstruction of undersampled data requires special techniques to avoid aliasing in the reconstructed images. Important parallel imaging techniques are sensitivity encoding (SENSE) [6], generalized autocalibrating partially parallel acquisition (GRAPPA) [7], and, especially for this thesis, regularized non-linear inverse reconstruction (NLINV) [9]. NLINV is described in more detail in Section 2.3.2

\subsection{Acquisition Sequences}

\subsubsection{FLASH}

Fast low-angle shot (FLASH) is a gradient-echo sequence first introduced by Frahm et al. [2]. It uses excitation pulses with small flip angles of less that $15^{\circ}$. Combined with fast (TR is usually less than $10 \mathrm{~ms}$ and often close to $2 \mathrm{~ms}$ ) and continuous imaging, this leads to the appearance of a steady-state, where the longitudinal magnetization lost to the 
excitation pulse is recovered over TR. This also explains the small flip angles: With larger flip angles, more of the longitudinal magnetization is available initially, leading to higher initial signals. However, as $T_{1}$ recovery over the short TR is not fast enough to build up a lot of magnetization, leaving less magnetization for the next excitation. Therefore, small flip angles often lead to higher steady state signal. The angle which maximizes the signal for given TR and $\left.T_{1}\right]$ is the Ernst angle $[18 \mathrm{pg} .430] \alpha_{E}=\arccos \left(\mathrm{e}^{-\mathrm{TR} / T_{1}}\right)$. For typical values of, for example, $\mathrm{TR}=3 \mathrm{~ms}$ and $T_{1}=1500 \mathrm{~ms}$, it is $\alpha_{E}=3.6^{\circ}$.

During such short TRs, even the transversal magnetization does not decay completely. Since this would have undesirable effects on the subsequent excitation, the transversal magnetization needs to be destroyed. This can be achieved by using strong gradients after readout, called crusher gradients, which destroy the transversal coherence. Another possibility is $\mathrm{rf}$ spoiling: here, the phase of the excitation pulse is changed in each TR, for example in a random manner [24]. This also prevents the buildup of transversal magnetization and does not need the time consuming crusher gradients.

\subsubsection{Balanced Steady-State Free Precession}

While residual transverse magnetization at the end of TR is an issue in FLASH it is exploited instead in balanced steady-state free precession (bSSFP) by balancing all gradients (slice selection gradient, readout gradient and possible phase-encoding gradients) no gradient dephasing of the transversal magnetization occurs. By applying the next excitation pulse with opposite phase (phase offset of $180^{\circ}$ ), this transversal magnetization can be reused. By applying successive pulses with $180^{\circ}$ phase offset and generating the echo at $\mathrm{TE}=\mathrm{TR} / 2$, the transversal magnetization at the end of TR resembles Figure 2.3 jumping from $+\alpha$ to $-\alpha$ and back. This sequence was first introduced by Carr [25] and a detailed overview over bSSFP can be found in Scheffler and Lehnhardt [26].

bSSFP is very attractive because of its increased signal compared to FLASH and because of its unique $T_{2} / T_{1}$ contrast. Especially in cardiac MRI. bSSFP is preferred because of its high contrast between blood and myocard [26].

However, the bSSFP sequence is very sensitive to off-resonance. This can understood in the following way: If a spin is off-resonant by $\Delta \omega$, it will precess by an angle $\theta=\Delta \omega \cdot$ TR in each TR. This leads to a smaller usable transverse magnetization in the next TR (compare Figure 2.3b) as the off-resonance increases. When $\theta=180^{\circ}$, the signal vanishes. A simulation showing the dependence of the signal on $\theta$ is shown in Figure 2.3 , showing the signal minima at $\theta= \pm 180^{\circ}$. These lead to the appearance of banding artifacts, which are characteristic for bSSFP. Examples of such banding artifacts are depicted in Figure $2.3 \mathrm{~d}$. 
The banding artifacts are generally smooth signal voids, as the signal curve smoothly depends $\theta$. As the off-resonance depends on TR, shorter TRs are desirable in bSSFP imaging.

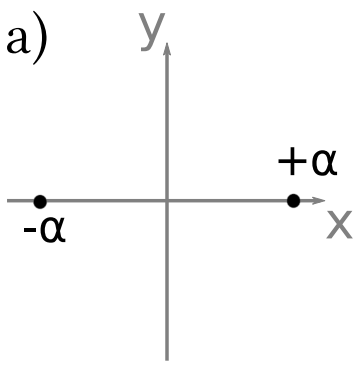

$\theta=0^{\circ}$

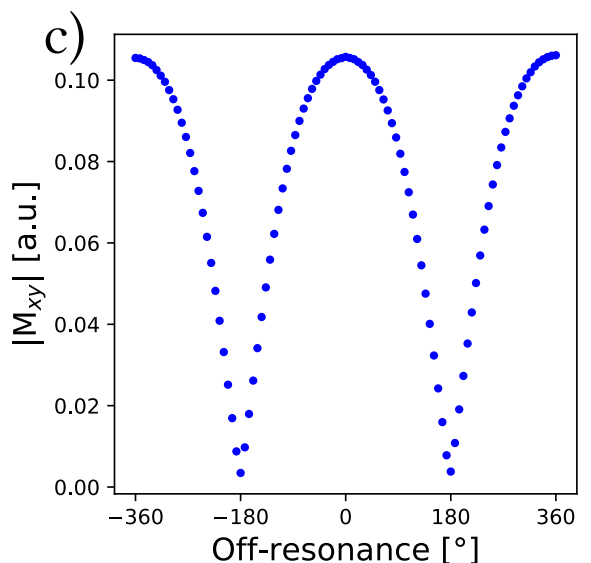

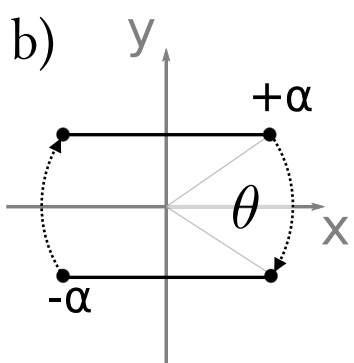

$\theta=75^{\circ}$

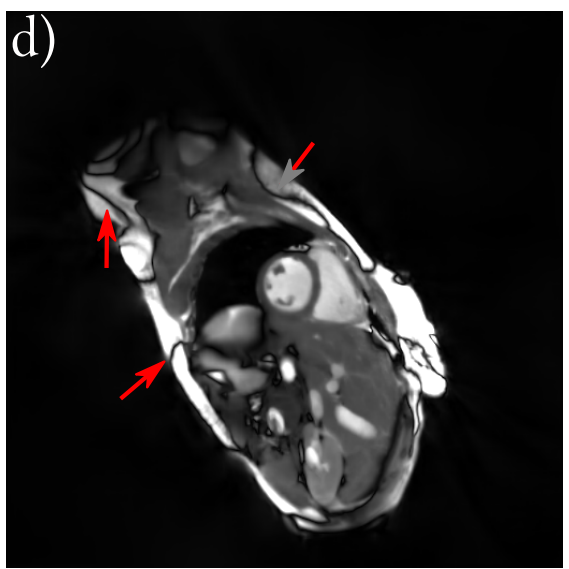

Figure 2.3: Off-resonance effects in bSSFP imaging. The motion of the magnetization during one TR is depicted for off-resonance angles of $\theta=0^{\circ}$ (a), and $\theta=75^{\circ}$ (b). (c): A simulation of the dependence of the signal on the phase offset $\theta$ for a flip angle of $70^{\circ}$ is shown, showing the characteristic signal minima at $\theta= \pm 180^{\circ}$. (d): Cardiac bSSFP image showing banding artifacts, indicated by red arrows. Diagram courtesy of Nick Scholand [27].

\subsection{Image Reconstruction}

\subsubsection{Parallel MRI as an Inverse Problem}

Mathematically, undersampled parallel MRI with known coil profiles can be understood as an inverse problem in the following way: If we are given an object with a certain spin density and a k-space trajectory, we can use Equation (2.3) to calculate the expected signal in k-space. However, given a signal evolution $s(\boldsymbol{k})$ in k-space and the trajectory, it 
is non-trivial to calculate the proton density $\rho(\boldsymbol{r})$ which gave rise to this signal. Therefore, the former is called the forward problem and the latter its inverse problem.

With known coil sensitivity profiles, Equation (2.3) can be rewritten in vector form.

$$
s=\mathcal{P F} \boldsymbol{F}
$$

where $\boldsymbol{s}$ is the vector of signals from all coils, $\mathcal{P}$ is the projection onto the acquired k-space trajectory, $\mathcal{F}$ is a $2 \mathrm{D}$ or $3 \mathrm{D}$ Fourier transform, $C$ is the vector of coil sensitivity profiles, and $\boldsymbol{\rho}$ is the spin density. $\mathcal{P}$ is necessary in undersampled MRI. as not all samples of the Fourier transform were measured.

In real settings, the measured signal will be corrupted by noise $\boldsymbol{n}$. Rewriting Equation 2.4] in the conventional form for inverse problems:

$$
\begin{gathered}
y=\mathcal{A} x+\boldsymbol{n} \\
\text { with } \mathcal{A}=\mathcal{P} \mathcal{F} C .
\end{gathered}
$$

Since $\mathcal{A}$ is composed of linear operations it is also linear. Equation 2.5 is therefore a linear inverse problem. This can be solved in a least-squares sense as

$$
\boldsymbol{x}^{*}=\underset{\boldsymbol{x}}{\arg \min }\|\boldsymbol{y}-\mathcal{A} \boldsymbol{x}\|_{2}^{2} .
$$

While this solution can, at times, be calculated directly with, for example, the pseudo inverse $\mathcal{A}^{+}=\left(\mathcal{A}^{H} \mathcal{A}\right)^{-1} \mathcal{A}^{H}$ of $\mathcal{A}$, the problem is often ill-conditioned and very large. Therefore, generally, regularization is added and the problem is solved iteratively [28], for example with the conjugate gradient method (CG)

\subsubsection{NLINV}

As we have seen, parallel MRI can be viewed as a linear inverse problem in cases where the coil sensitivities are either known or identically 1 (in the case of a single coil). However, with unknown coil profiles, this can also be viewed as a non-linear inverse problem:

$$
\left(\boldsymbol{x}^{*}, C^{*}\right)=\underset{(\boldsymbol{x}, C)}{\arg \min }\|\boldsymbol{y}-\mathcal{P} \mathcal{F} C \boldsymbol{x}\|_{2}^{2} .
$$

where the non-linearity presents as the product of the two unknowns, the image $x$ and the coil sensitivity profiles $C$. This approach has first been formulated as Joint Image Reconstruction and Sensitivity Estimation in SENSE (JSENSE) [29] and regularized nonlinear inverse reconstruction (NLINV) [9, 21]. These approaches are useful since they simultaneously estimate the coil profiles from the same data, thereby not requiring a pre-processing step. This also makes better use of all available data compared to linear parallel imaging approaches, which estimate the coil profiles in a separate step from a calibration region, which is just a subset of the data. 
Equation 2.6 can also be cast as a non-linear operator equation

$$
F(x)=y, \quad \text { with } x=\left(\begin{array}{c}
\rho \\
c_{1} \\
\vdots \\
c_{N}
\end{array}\right)
$$

with a non-linear operator $F$ mapping the $N$ unknown coil profiles and the unknown proton density onto the measured k-space data $y$ :

$$
F: x \mapsto\left(\begin{array}{c}
\mathcal{P F} c_{1} \rho \\
\vdots \\
\mathcal{P F} c_{N} \rho
\end{array}\right)
$$

In this form Equation 2.7 can be solved using the iteratively regularized Gauss-Newton method (IRGNM) (see [21. Chapter 5]). The IRGNM work by linearizing the non-linear problem around the current guess $x_{n}$

$$
F\left(x_{n}+\mathrm{d} x\right) \approx D F\left(x_{n}\right) \mathrm{d} x+F\left(x_{n}\right)
$$

and solving that linearized subproblem with a suitable algorithm. Here, $D F\left(x_{n}\right)$ is the Jacobian of $F$ at $x_{n}$. It is iteratively regularized in that the regularization is reduced in each outer iteration (called Newton step). In this way, the regularization is large in the beginning, when the reconstruction is still far from solution, and small when it is close to the solution. This makes the algorithm robust when it is far from a solution, and more accurate when it gets closer. The linearized inner problem can be solved, for example, by the conjugate gradient method (CG)

A problem in Equation 2.8 is the lack of separation between image and coil profiles: if each $c_{i}$ is multiplied by a complex function and $\rho$ divided by the same function, the result of the operator is unchanged. This can even be taken to the extreme: with $\rho \equiv 1$, all proton density information can be absorbed into the coil profiles. To counteract this, prior knowledge about the smoothness of the coil profiles can be used: by adding a regularization term penalizing high spatial frequencies, for example by including a suitable Sobolev norm, this symmetry can be broken. This has been shown to be sufficient to recover proper images and coil profiles [21].

NLINV] was also extended for real-time reconstruction [8, 30], by including a regularization with respect to the previous frame. If the frame rate of the dataset is high enough to resolve the dynamics of the measured object, this difference is bound to be small. 


\subsubsection{Compressed Sensing}

A recent innovation in MRI is the introduction of compressed sensing (CS) by Block, Uecker, and Frahm [10] and Lustig, Donoho, and Pauly [11]. It enables reconstruction from undersampled data even in the case of a single coil, that means without exploiting parallel imaging. It is based on the fact that transformations $\Psi$ exist such that the number of nonzero components of an $n \times n$ MRI image $x,\|\Psi x\|_{0}$, is much less than $n \times n$. Examples for such sparsifying transforms are the wavelet transform or the computation of finite differences as used in the total variation semi-norm.. This, however, implies that identifying a small number of non-zero values of $\Psi x$ can be enough to recover an image, suggesting a great potential for undersampling. Although the transformed samples cannot be measured directly, it can be shown that under certain assumptions on the measurement operator $\mathcal{A}$ an image can be reconstructed by minimizing

$$
\begin{gathered}
\underset{x}{\arg \min }\|\Psi x\|_{0} \\
\text { subject to }\|y-\mathcal{A} x\|_{2}^{2}<\varepsilon
\end{gathered}
$$

subject to a data consistency constraint with a certain desired accuracy $\varepsilon$. In practice, to solve large reconstruction problems one minimizes $\|\cdot\|_{1}$ instead of $\|\cdot\|_{0}$ as in

$$
\begin{gathered}
\underset{x}{\arg \min }\|\Psi x\|_{1} \\
\text { subject to }\|y-\mathcal{A} x\|_{2}^{2}<\varepsilon
\end{gathered}
$$

which, surprisingly, yields the same results [31]. Although convex, Equation 2.10 is not smooth, since the $\ell_{1}$-norm is non-differentiable. Algorithms that can solve these non-smooth optimization problems are, for example, fast iterative shrinkage/thresholding algorithm (FISTA) [32] and alternating direction methods of multipliers (ADMM) 33, 34].

To make recovery of the sparse coefficients from undersampled data possible, a certain incoherency condition between the measurement basis and the sparse representation has to be fulfilled [35]. In MRI one possibility is radial imaging, where undersampling leads to the appearance of streak artifacts, which can be considered incoherent. In $3 \mathrm{D}$ Cartesian acquisitions, incoherent undersampling can be achieved by randomizing acquisitions in the two phase-encoding directions, for example by utilizing Poisson-disc sampling [36]. 


\section{MRI System and Hardware}

MRI Scanner As explained in Section 2.1 MRI needs a strong static magnetic field, timevarying gradient fields in arbitrary directions, and coils for $\mathrm{rf}$ excitation and data recording. The MRI system used for most of the data in this thesis is a Siemens MAGNETOM Skyra whole body human MRI scanner (Siemens Healthineers AG, München, Germany), with a $2.89 \mathrm{~T} B_{0}$ field The superconducting main magnet is cooled with liquid helium.

A system of gradient coils provides arbitrary magnetic field gradients with a maximum strength of $45 \frac{\mathrm{mT}}{\mathrm{m}}$ and a maximum slew rate of $200 \mathrm{~T} / \mathrm{m} / \mathrm{s}$

A single transmit coil for excitation is integrated into scanner. For data acquisition and to exploit parallel imaging, different coils for different applications are available: A 30-channel thorax coil, with part of the coil segments integrated into the patient table, can be used for cardiac and abdominal MRI while a 20 -channel and a 64-channel head coil are available for imaging of the head and the brain.

Reconstruction Hardware For this thesis, image reconstruction was performed away from the scanner on separate computers. The first system used is a Supermicro SuperServer (Supermicro Inc., San Jose, USA) with 2 Intel Xeon E5-2650 v3 ten-core CPUs at $2.30 \mathrm{GHz}$ with $512 \mathrm{GiB}$ RAM, running Debian 9.9 "Stretch"2 The second system is a Dell Optiplex 7020 (Dell Inc., Round Rock, USA) with a single Intel Core) i5-459o four-core CPU at $3.30 \mathrm{GHz}$ and $16 \mathrm{GiB}$ RAM also running Debian 9.9 "Stretch".

Reconstruction Software Multiple reconstruction methods were implemented during the course of this thesis. For quick prototyping and for fast reconstruction, a C-based toolbox commonly used for parallel and compressed sensing MRI was chosen: The Berkeley Advanced Reconstruction Toolbox (BART) 37.

This toolbox contains efficient and parallelized versions of commonly needed operations, such as matrix-matrix and matrix-vector multiplications, fast (discrete) Fourier

${ }^{1}$ This is still referred to as a $3 \mathrm{~T}$ system 2https://www.debian.org// 
transforms (FFTs) and the non-uniform fast Fourier transform (nuFFT), sparsifying transforms such as different types of wavelets and decompositions such as the singular value decomposition (SVD) or an eigendecomposition. It does not implement all of these itself, instead relying on tested libraries such as LAPACK $3^{3}$ and FFTW 4 Furthermore, it already includes generic implementations of commonly used algorithms, such as the conjugate gradient method (CG), the fast iterative shrinkage/thresholding algorithm (FISTA), the alternating direction methods of multipliers (ADMM) and the iteratively regularized Gauss-Newton method (IRGNM), It also includes implementations of common MRI methods, including ESPIRiT, SENSE and simultaneous autocalibrating and k-space estimation (SAKE),

Apart from the application programming interface (API) which was used to implement the methods described in this thesis, it also provides a set of command-line tools for interactive use.

BART is a widely used software package in MRI research. As it is written in C11 with GNU extensions, it is easily portable to different computer systems which are capable of running the GNU compiler collection (GCC) 5 


\section{Rank-relaxed MRI Signal Model for Robust Reconstruction}

The following section will introduce a non-linear method based on a higher-dimensional, relaxed signal model named extended non-linear inversion based on ESPIRiT (ENLIVE) ENLIVE has been published in Scientific Reports and the corresponding manuscript is reprinted as Section 4.1. Applications of ENLIVE for the calculation of high-quality coil profiles and for robust reconstruction of real-time data are shown in Section 4.2 and Section $4 \cdot 3$, respectively.

\subsection{ENLIVE: An Efficient Nonlinear Method for Calibrationless and Robust Parallel Imaging}

The following is a reprint of the original article

H. C. M. Holme, S. Rosenzweig, F. Ong, R. N. Wilke, M. Lustig, and M. Uecker. "ENLIVE: An Efficient Nonlinear Method for Calibrationless and Robust Parallel Imaging”. Sci. Rep. 9 (2019), p. 3034. DOI:10.1038/s41598-019-39888-7

$H C M H$, SR and $M U$ implemented the method. $H C M H$ performed the numerical experiments. $H C M H, R N W$ and $M U$ contributed to the data analysis. $F O$ and $M L$ provided guidance on design and implementation. $H C M H$ wrote the majority of the manuscript and its revisions. All authors contributed to the preparation of the manuscript. 


\section{Abstract}

Robustness against data inconsistencies, imaging artifacts and acquisition speed are crucial factors limiting the possible range of applications for magnetic resonance imaging (MRI). Therefore, we report a novel calibrationless parallel imaging technique which simultaneously estimates coil profiles and image content in a relaxed forward model. Our method is robust against a wide class of data inconsistencies, minimizes imaging artifacts and is comparably fast combining important advantages of many conceptually different state-of-the-art parallel imaging approaches. Depending on the experimental setting, data can be undersampled well below the Nyquist limit. Here, even high acceleration factors yield excellent imaging results while being robust to noise and the occurrence of phase singularities in the image domain, as we show on different data. Moreover, our method successfully reconstructs acquisitions with insufficient field-of-view. We further compare our approach to ESPIRiT and SAKE using spin-echo and gradient echo MRI data from the human head and knee. In addition, we show its applicability to non-Cartesian imaging on radial FLASH cardiac MRI data. Using theoretical considerations, we show that ENLIVE can be related to a low-rank formulation of blind multi-channel deconvolution, explaining why it inherently promotes low-rank solutions. 


\subsubsection{Introduction}

Since acquisition speed is a major issue in $\mathrm{MRI}$ accelerated imaging with multiple receiver coils has been an active field of research since its beginning. Quite rapidly, two main categories of parallel imaging methods emerged: sensitivity encoding (SENSE) [6] is the prototypical example and k-space methods, where it is generalized autocalibrating partially parallel acquisition (GRAPPA) [7]. SENSE-like methods, when the coil sensitivity profiles are known, permit a natural description as a linear inverse problem. Incorporating the estimation of coil sensitivity profiles into the reconstruction leads to a nonlinear inverse problem, as formulated in Joint Image Reconstruction and Sensitivity Estimation in SENSE (JSENSE) [29] and regularized non-linear inverse reconstruction (NLINV) [9].

Additionally, low-rank and subspace methods [39-42] have been proposed to further increase reliability and acceleration in MRI These methods exploit prior knowledge on the structure of the matrices arising in MRI reconstruction. Recently, ESPIRiT [15] has been shown to provide robustness towards data inconsistencies similar to k-space methods such as GRAPPA [7]. In particular, in cases where the chosen field-of-view (FOV) is smaller than the object [43] and in phase-constraint imaging [44], it was shown that methods based on traditional SENSE that only use a single set of coil sensitivity profiles exhibit artifacts. In ESPIRiT, robust reconstruction is possible through a relaxed SENSE-model, which uses multiple images and sets of coil sensitivity profiles.

ESPIRiT recovers accurate coil sensitivities using an eigenvalue decomposition of an image-domain operator which projects onto the signal space of the calibration matrix. In case of inconsistencies, it produces multiple sets of maps which can be used in a relaxed SENSE reconstruction. ESPIRiT requires a fully-sampled calibration region in the center of k-space. Additionally, it cannot be applied directly to non-Cartesian data, requiring an additional gridding step to generate calibration data. A more generic subspace method is SAKE [39], because it can be directly applied to data without fully-sampled calibration region or non-Cartesian data. Based on the idea that the signal is contained in a subspace of smaller dimensionality which can be recovered, SAKE uses structured low-rank matrix completion to recover a full $\mathrm{k}$-space from incomplete data. Unfortunately, it is computationally extremely demanding as each iteration has to perform a singularvalue decomposition (SVD). Furthermore, because it operates completely in k-space, regularization terms may require additional Fourier transforms and must be applied to all channels. Calibration-free locally low-rank encouraging reconstruction (CLEAR) [42] is a related method which uses local low-rankness in the image domain instead of the global k-space rank penalty used in SAKE This reduces the computational complexity 
by reducing the size of the needed SVDs, although it does increase the number of SVDs necessary. Furthermore, as it is an image space method, regularization can be integrated more easily.

Regularized Nonlinear Inversion (NLINV) [9] jointly estimates the image content and the coil sensitivity profiles using a nonlinear algorithm. Similar to SAKE it does not require a fully-sampled Cartesian calibration region and can be applied directly to non-Cartesian data.

This work aims at combining the advantages from these different methods. Inspired by ESPIRiT, we propose an extension to NLINV that extends it beyond the original SENSElike model. This method, termed ENLIVE(Extended NonLinear InVersion inspired by ESPIRiT), can be related to a convex relaxation of the NLINV problem subject to a lowrank constraint. From NLINV it inherits its flexibility and suitability for calibrationless and non-Cartesian imaging; from ESPIRiT it inherits robustness to data inconsistencies. We apply ENLIVE to several imaging settings covering limited FOV, phase constraints, phase singularities, and non-Cartesian acquisition. Additionally, we present comparisons to ESPIRiT and to SAKE,

Initial results have been presented at the $25^{\text {th }}$ Annual Meeting of the International Society for Magnetic Resonance in Medicine [45].

\subsubsection{Theory}

\section{Formulation}

NLINV recovers the image $\boldsymbol{m}$ and the coil sensitivity profiles $\boldsymbol{c}_{j}$ from measurements $\boldsymbol{y}_{j}$ by solving the regularized nonlinear optimization problem:

$$
\underset{\boldsymbol{m}, \boldsymbol{c}_{j}}{\arg \min } \sum_{j=1}^{N_{C}}\left\|\boldsymbol{y}_{j}-\mathcal{P} \mathcal{F}\left\{\boldsymbol{c}_{j} \odot \boldsymbol{m}\right\}\right\|_{2}^{2}+\alpha\left(\sum_{j=1}^{N_{C}}\left\|\boldsymbol{W} \boldsymbol{c}_{j}\right\|_{2}^{2}+\|\boldsymbol{m}\|_{2}^{2}\right)
$$

with $N_{C}$ coils, the two or three dimensional Fourier $\operatorname{transform} \mathcal{F}$, the projection $\mathcal{P}$ onto the measured trajectory (or the acquired pattern in Cartesian imaging) and an invertible weighting matrix $W$ penalizing high frequencies in the coil profiles. Here, both image $\boldsymbol{m} \in C^{n_{x} \cdot n_{y} \cdot n_{z}}$ and coils $\boldsymbol{c}_{j} \in C^{n_{x} \cdot n_{y} \cdot n_{z}}$ are regarded as vectors of size $n_{x} \cdot n_{y} \cdot n_{z}=: N_{I}$ and $\odot$ is their element-wise product.

In this work, we propose to extend this model to:

$$
\underset{\boldsymbol{m}^{i}, \boldsymbol{c}_{j}^{i}}{\arg \min } \sum_{j=1}^{N_{C}}\left\|\boldsymbol{y}_{j}-\mathcal{P} \mathcal{F}\left\{\sum_{i=1}^{k} \boldsymbol{c}_{j}^{i} \odot \boldsymbol{m}^{i}\right\}\right\|_{2}^{2}+\alpha \sum_{i=1}^{k}\left(\sum_{j=1}^{N_{C}}\left\|\boldsymbol{W} \boldsymbol{c}_{j}^{i}\right\|_{2}^{2}+\left\|\boldsymbol{m}^{i}\right\|_{2}^{2}\right)
$$

where $c_{j}^{i}$ and $\boldsymbol{m}^{i}$ are $k$ sets of unknown coil sensitivity profiles and unknown images. 
This approach is inspired by ESPIRiT, which uses additional maps to account for model violations [15].

In the following, we will show that this formulation automatically produces solutions with rank even smaller than $k$ if one exits. To show this, we first relate Equation 4.2 to a linear inverse problem for matrices with nuclear norm regularization.

From here on, we assume that the variable transformation $\hat{\boldsymbol{c}}_{j}=\boldsymbol{W} \boldsymbol{c}_{j}$ has been applied to move the weighting matrix from the regularization into the forward operator. We note that this problem is equivalent to a corresponding multi-channel blind deconvolution problem [46] in k-space via the convolution theorem. Using the "lifting" approach used for such blind deconvolution problems [47], which can also be applied in the image domain, we now lift the Equation (4.1) into a linear inverse problem in terms of a rank-1 matrix $\boldsymbol{X}=\boldsymbol{u} \boldsymbol{v}^{T}$ formed by the tensor product of $\boldsymbol{u}$ and $\boldsymbol{v}$, where $\boldsymbol{u}$ corresponds to $\boldsymbol{m}$ and $\boldsymbol{v}$ is a stacked vector composed of the weighted coil sensitivity profiles $\hat{\boldsymbol{c}}_{j}$. The problem then becomes:

$$
\underset{\boldsymbol{u}, \boldsymbol{v}}{\arg \min }\left\|\boldsymbol{y}-\mathcal{A}\left\{\boldsymbol{u} \boldsymbol{v}^{T}\right\}\right\|_{2}^{2}+\alpha\left(\|\boldsymbol{u}\|_{2}^{2}+\|\boldsymbol{v}\|_{2}^{2}\right)
$$

with a linear operator $\mathcal{A}$ mapping $\boldsymbol{u} \boldsymbol{v}^{T}$ to $\mathcal{P} \mathcal{F} \boldsymbol{c}_{j} \odot \boldsymbol{m}$ and a vector $\boldsymbol{y}$ containing measurement data of all coils. Such an $\mathcal{A}$ exists because $\boldsymbol{u} \boldsymbol{v}^{T}$ contains all possible products of elements of $\boldsymbol{u}$ and $\boldsymbol{v}$. Its explicit action is explained in more detail in the Appendix. In general, all bilinear functions can be expressed as linear functions on the tensor product of the two vector spaces involved.

As suggested by Ahmed et al. [47] for blind multi-channel deconvolution, we now relax the rank-1 constraint and allow $k$ sets of images and coil sensitivity profiles. This corresponds to using $\boldsymbol{X}=\boldsymbol{U} \boldsymbol{V}^{T} \in C^{N_{I} \times N_{C} \cdot N_{I}}$ with $\boldsymbol{U} \in C^{N_{I} \times k}$ and $\boldsymbol{V} \in C^{N_{C} \cdot N_{I} \times k}$, which then leads to the optimization problem

$$
\underset{\boldsymbol{U}, \boldsymbol{V}}{\arg \min }\left\|\boldsymbol{y}-\mathcal{A}\left\{\boldsymbol{U} \boldsymbol{V}^{T}\right\}\right\|_{2}^{2}+\alpha\left(\|\boldsymbol{U}\|_{F}^{2}+\|\boldsymbol{V}\|_{F}^{2}\right)
$$

with the Frobenius norm $\|\cdot\|_{F}$. In the Appendix we show how this corresponds to ENLIVE as formulated in Equation 4.2. Under conditions given below, Equation 4.4 is equivalent to a convex optimization problem for the matrix

$$
\underset{\boldsymbol{X}}{\arg \min }\|\boldsymbol{y}-\mathcal{A}\{\boldsymbol{X}\}\|_{2}^{2}+2 \alpha\|\boldsymbol{X}\|_{\star}
$$

with nuclear norm $\|\cdot\|_{\star}$ regularization [48, 49]. The nuclear norm promotes low-rank solutions. Furthermore, if the solution to Equation (4.5) has rank smaller than or equal to $k$ both problems are equivalent in the sense that from a solution $U, V$ of Equation 4.4 ) one obtains a solution of Equation 4.5 via $X=U V^{T}$ which attains the same value and from 
a solution $X$ of Equation (4.5) one can construct a solution of Equation (4.4) that attains the same value. This is achieved by factorizing $X$ using the SVD and by distributing the singular values in an optimal way, i.e. equally as square roots, to the two factors. Please note that we do not propose to use this convex formulation for computation as it is very expensive, instead we propose to use the nonlinear formulation given in Equation 4.2 . Nevertheless, this relationship to nuclear-norm regularization is important as it explains why ENLIVE produces solutions with low rank even smaller than $k$, if one exists.

\section{Implementation}

Similar to NLINV [9], we solve Equation (4.2) using the iteratively regularized GaussNewton method (IRGNM). The IRGNM solves successive linearizations with the regularization parameter decreasing in each Newton step: Starting from $\alpha_{0}$, the regularizations in each step is reduced according to $\alpha_{n}=\alpha_{0} q^{n-1}, 0<q<1$. As initial guess, we use $\boldsymbol{m}^{i} \equiv 1$ for the images and $\boldsymbol{c}_{j}^{i} \equiv 0$ for the coil sensitivity profiles. Because we initialize images and sensitivity profiles for all sets in the same way, the problem is symmetric in the sets and the algorithm will produce degenerate solutions with identical sets. To break this symmetry, we require the $k$ sets of coil profiles to be orthogonal using Gram-Schmidt orthogonalization after each Newton step. For orthogonalization, the coil profiles of each set are treated as stacked one-dimensional vectors.

The weighting matrix $\boldsymbol{W}$ enforcing smoothness in the coil profiles was chosen as in [9]. In k-space, this leads to a penalty increasing with distance from the center of k-space according to $\left(1+a\|\boldsymbol{k}\|^{2}\right)^{b / 2}$. In this work, $a=240$ and $b=40$ were used. Furthermore, $\mathrm{k}$-space is normalized so that it extends from $-n_{i} / 2$ to ${ }^{n_{i}} / 2$ for $i \in\{x, y, z\}$. As $W$ applies weights in k-space, it is the product of a Fourier matrix transforming each coil profile to k-space an of this diagonal weight matrix.

Images and coil profiles are combined in a post-processing step. This is used to either create individual images for each set $i$ by combining coil-weighted images $\boldsymbol{m}^{i} \boldsymbol{c}_{j}^{i}$ using

$$
\boldsymbol{M}^{i}=\sqrt{\sum_{j=1}^{N_{C}}\left|\boldsymbol{m}^{i} \odot \boldsymbol{c}_{j}^{i}\right|^{2}}
$$

or to create a single combined image by first combining each set to obtain a proper image for each coil and then doing a final coil combination with

$$
\boldsymbol{M}=\sqrt{\sum_{j=1}^{N_{C}}\left|\sum_{i=1}^{k} \boldsymbol{m}^{i} \odot \boldsymbol{c}_{j}^{i}\right|^{2}} .
$$




\subsubsection{Results}

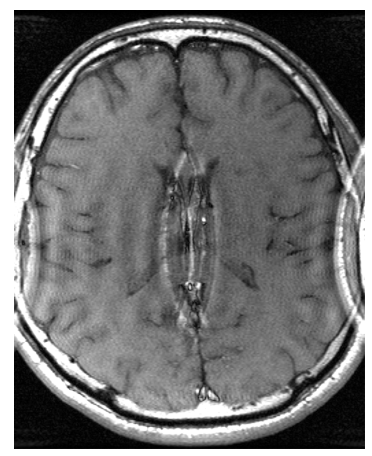

1 map

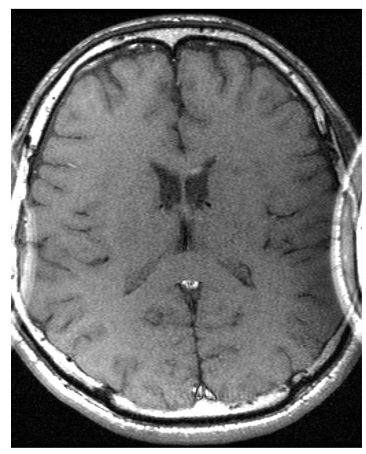

map \#1

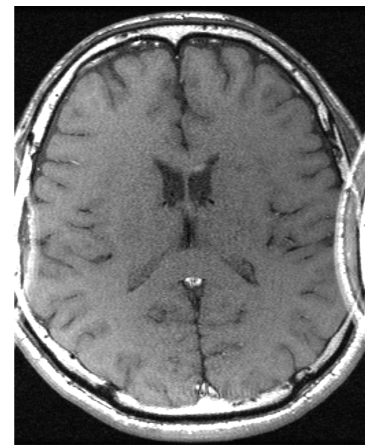

2 maps

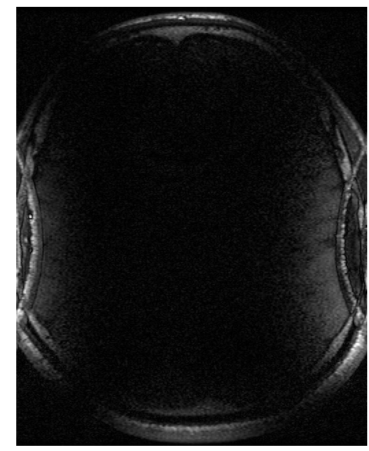

map \#2

\section{ENLIVE}

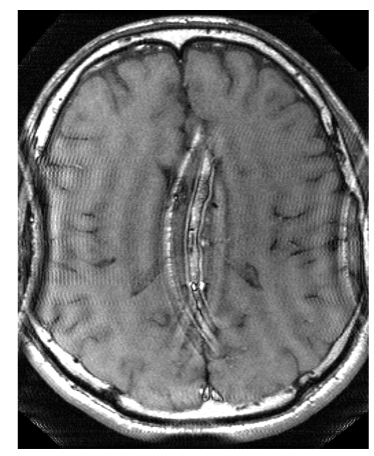

1 map

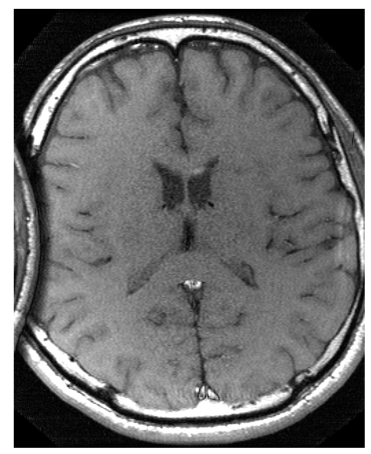

map \#1

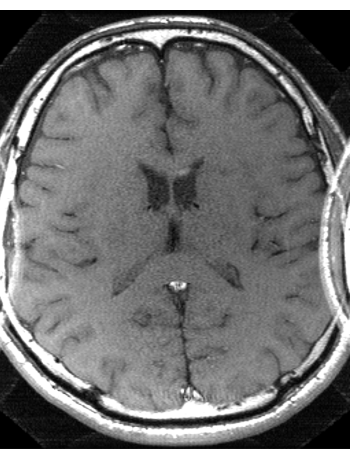

2 maps

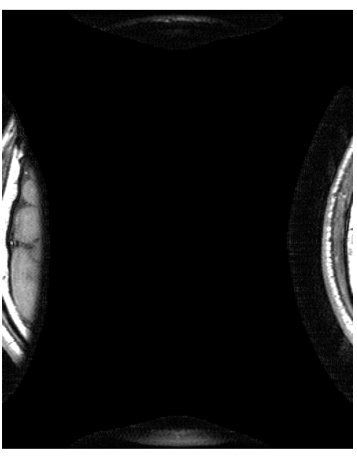

map \#2

\section{ESPIRiT}

Figure 4.1: Comparison of ENLIVE and ESPIRiT reconstructions allowing both one and two sets of maps (top row) together with individual map images (bottom row) for the reconstructions using two maps. While the reconstructions using a single set of maps exhibit strong aliasing artifacts, the reconstructions allowing two sets of maps are artifact-free. The reason can be seen in the individual images: A single image with a single set of coil profiles cannot resolve the aliasing arising from the infolded sides. Using two sets of maps, the region causing infolding can be separated into the second image.

\section{Limited FOV}

In the examples with a restricted FOV, both ENLIVE with a single set of maps, i.e. NLINV and ESPIRiT reconstructions show a similar central artifact (Figure 4.1). This artifact can be readily explained as a consequence of the undersampling pattern and the signal model violation at the edges of the image: Without a parallel imaging reconstruction, we expect 

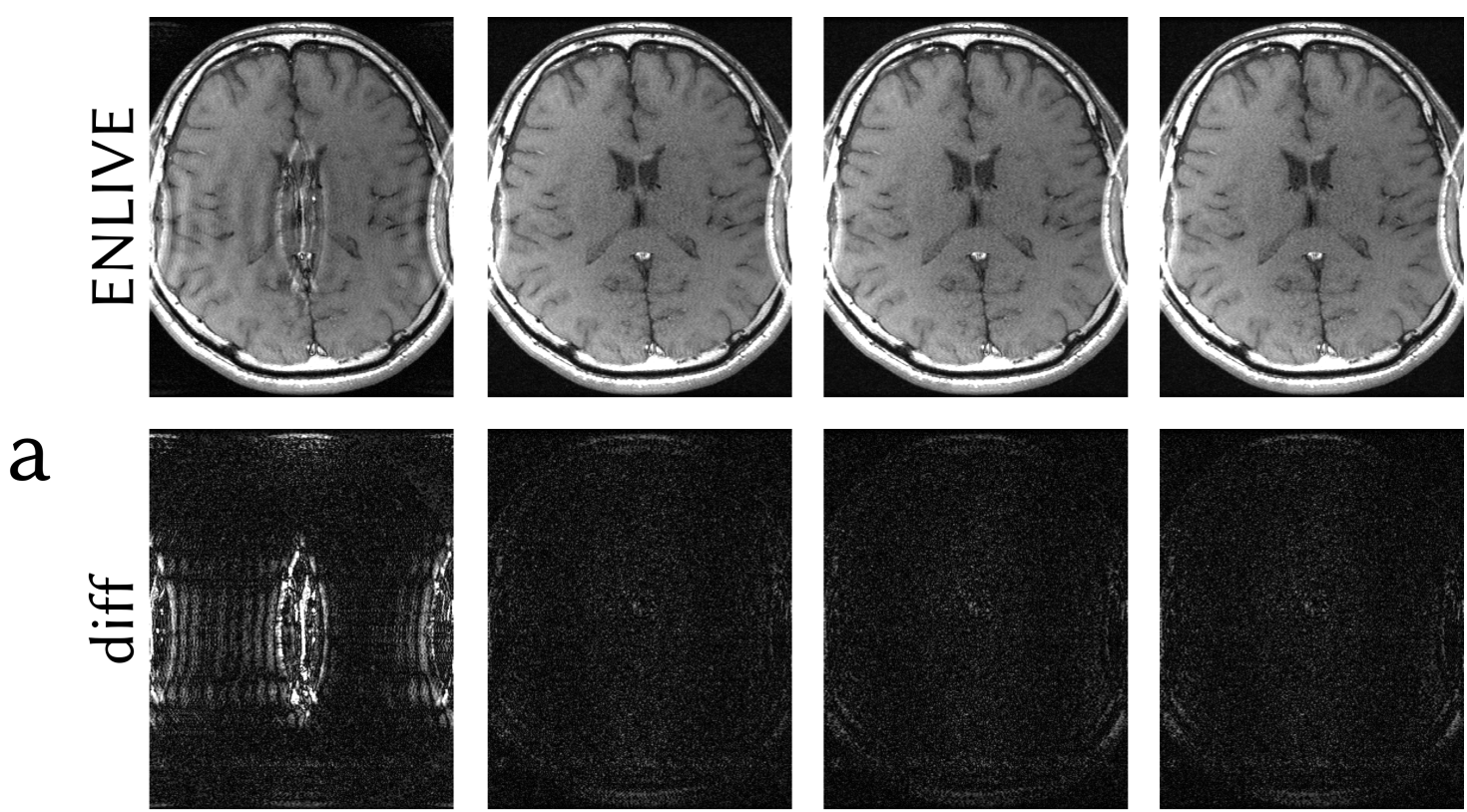

1 map

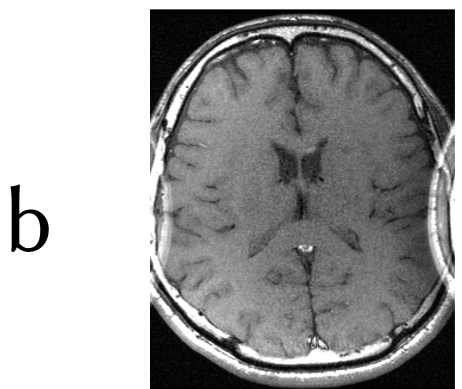

map \#1
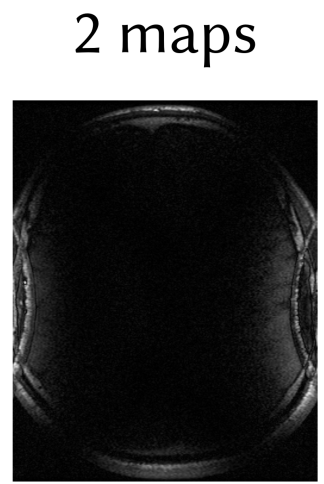

map \#2
3 maps

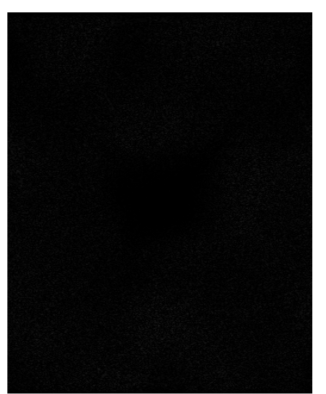

map \#3
4 maps

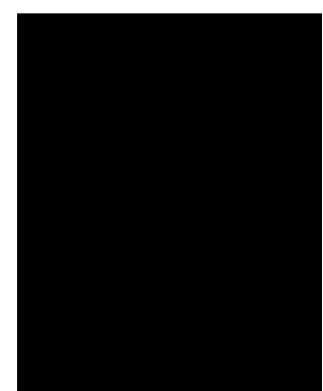

map \#4

Figure 4.2: (a): ENLIVE reconstructions of the same data as in Fig. 4.1 using 1, 2, 3 and 4 sets of maps. Difference images to fully-sampled reference data are shown in the bottom row. Using a single map, the central artifact is clearly visible in the reconstruction as well as in the difference image. Using 2 and more maps, the artifact is resolved and the difference images show close to no variation. (b): Individual map images of the reconstruction using 4 maps. Since 2 sets of maps are sufficient to fully describe the data, the first two maps are similar to the maps depicted in Fig. 4.1 while maps 3 and 4 are close to zero. The corresponding coils profiles are depicted in Fig. $4 \cdot 3$ 


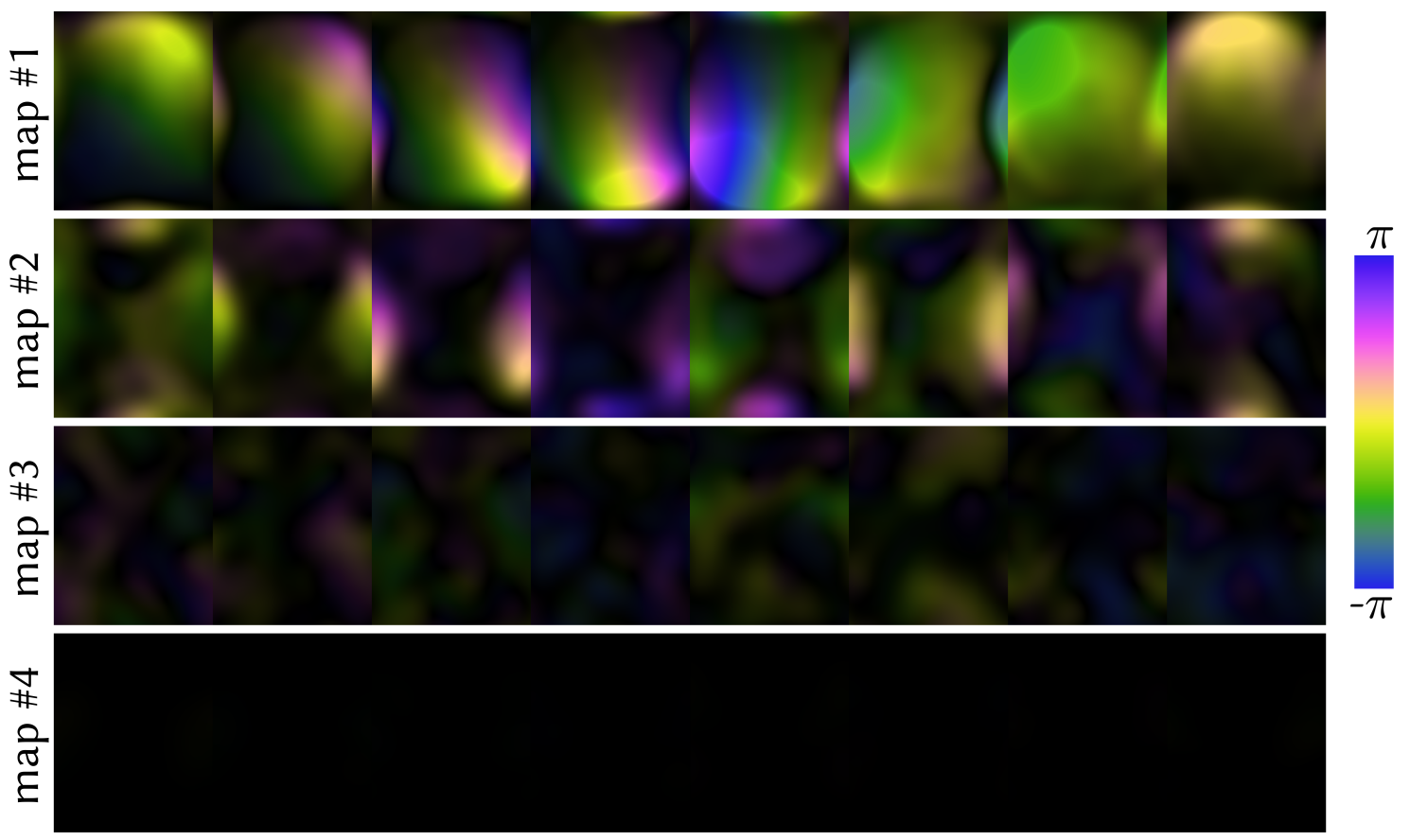

Figure 4.3: Calculated coil sensitivity profiles for the ENLIVE reconstruction using 4 sets of maps shown in Fig. 4.2 The second map is sensitive in the region which causes infolding in the single-map reconstruction, while the first map is smoothly sensitive over the entire FOV. The third and fourth map show very little sensitivity anywhere. Magnitude is encoded in brightness while phase in encoded in the color, according to the cyclic magenta-yellow-green-blue colormap described in [50]. 

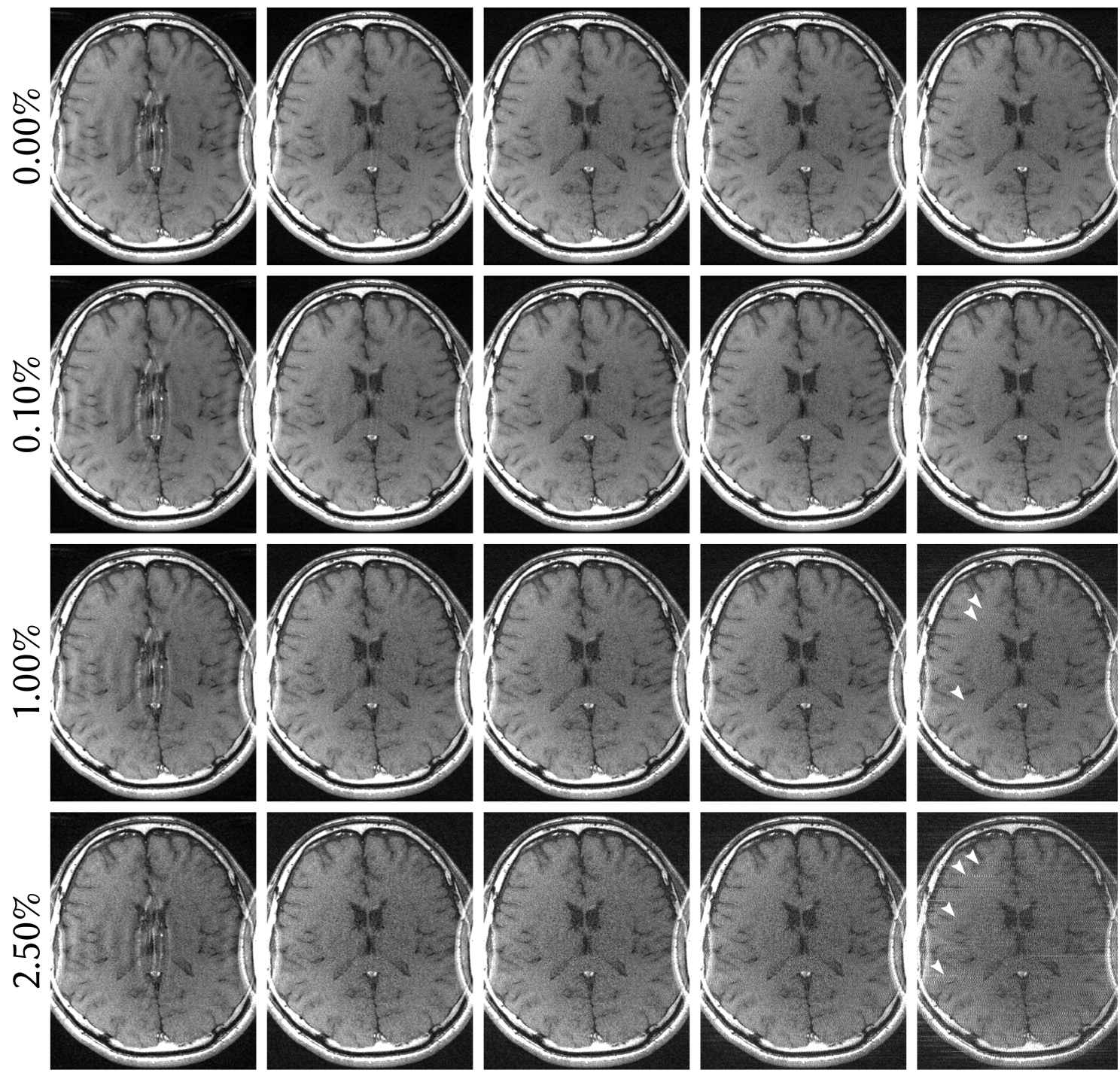

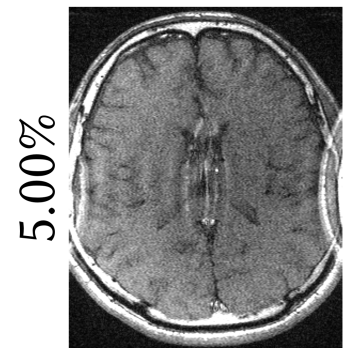

13

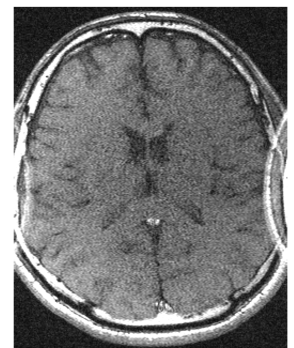

16

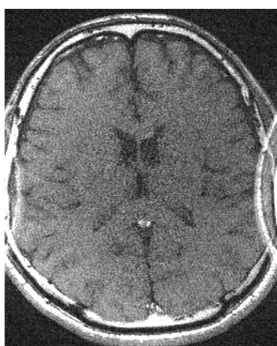

19

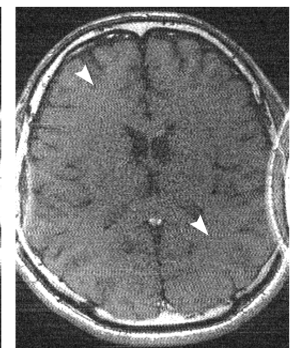

22

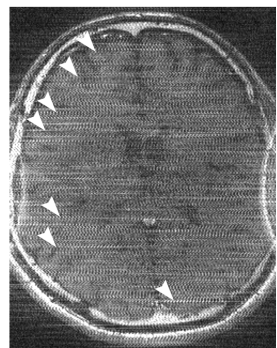

25

Figure 4.4: ENLIVE reconstruction with 2 maps with differing number of Newtons steps (left to right) and different levels of added noise (top to bottom) of the same dataset as in Figure 4.1 Gaussian white noise was added to the $\mathrm{k}$-space before reconstruction. The standard deviation of the added noise was varied between o and $5 \%$ of the absolute value of the DC component. Using too few Newton steps leads to residual infolding artifacts, while too many Newton steps cause high-frequency artifacts to appear (some of which are indicated by arrows). Since the number of Newton steps controls the regularization in the IRGNM, we can understand these two effects as too much and too little regularization. In all cases, the added noise has no impact on the infolding artifact. 

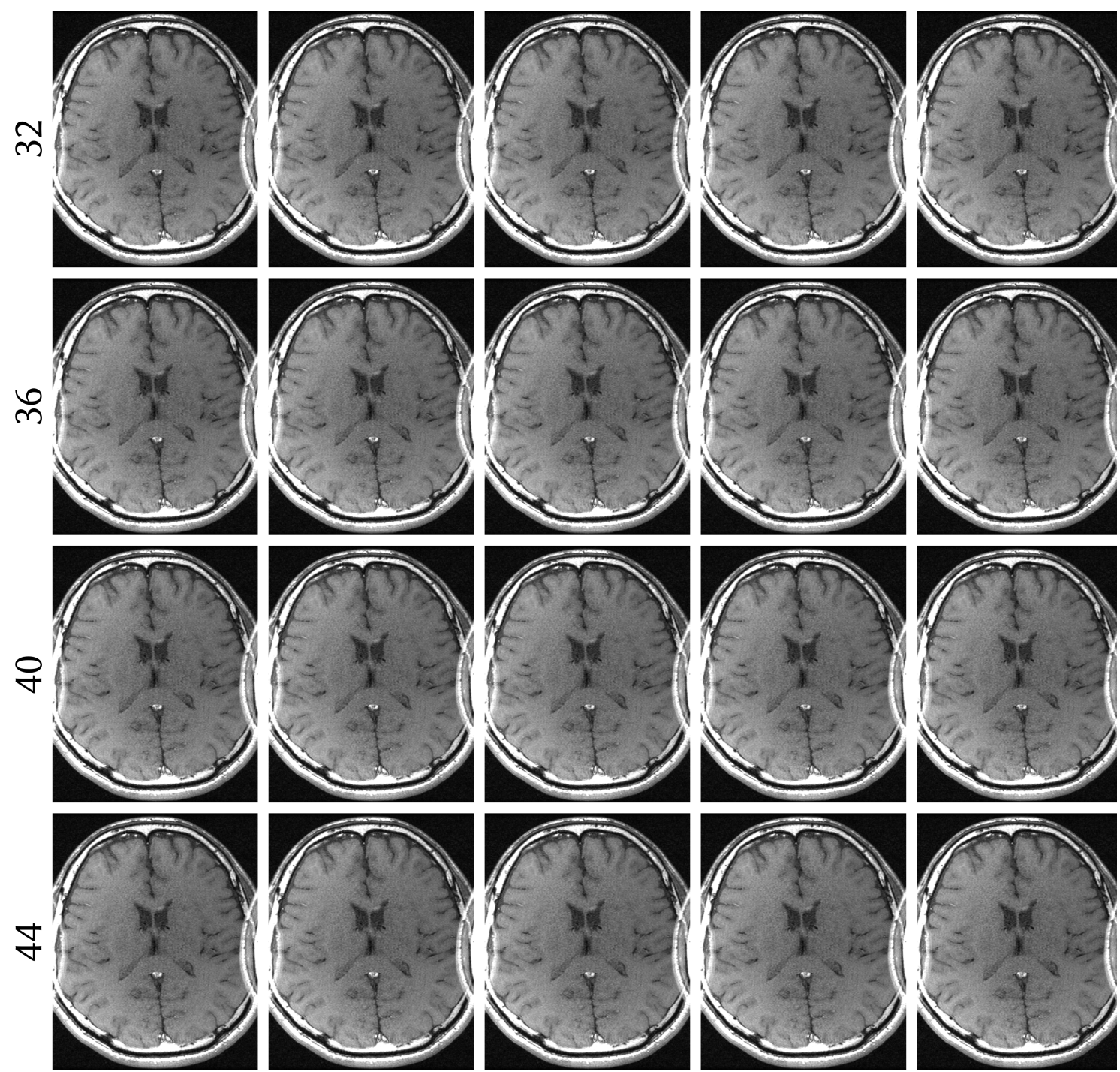

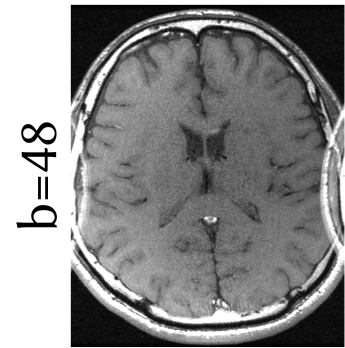

$\mathrm{a}=200$

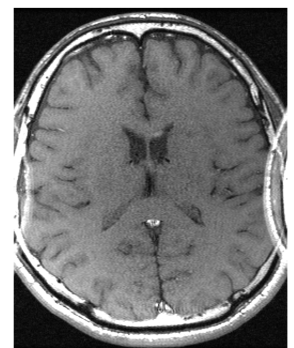

220

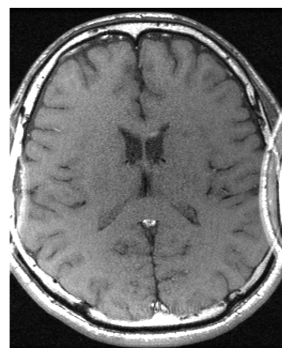

240

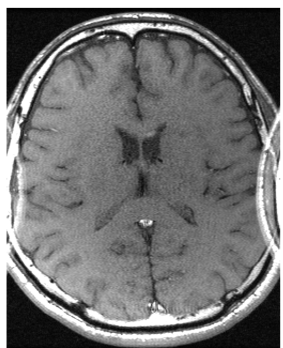

260

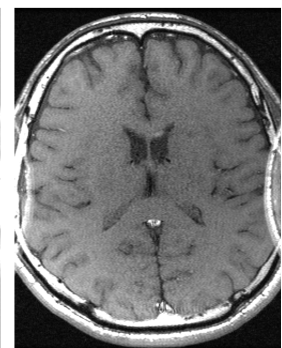

280

Figure 4.5: ENLIVE reconstruction with 2 maps of the same dataset as in Figure 4.1 with different parameters for the coil weighting matrix $W$. $W$ applies a penalty in k-space according to $\left(1+a\|k\|^{2}\right)^{b / 2}$. $a$ varies from left to right while $b$ varies from top to bottom. For all other reconstructions, $a=240$ and $b=40$ (center image) were used. The infolding artifact does not appear for any parameter pair, indicating that the reconstruction is not sensitive to specific values of $a$ or $b$. 


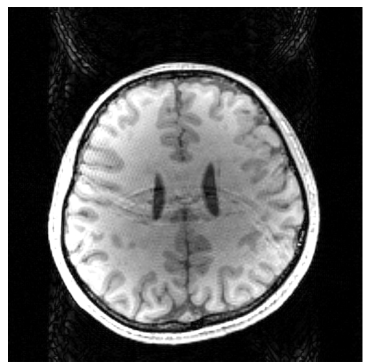

1 map

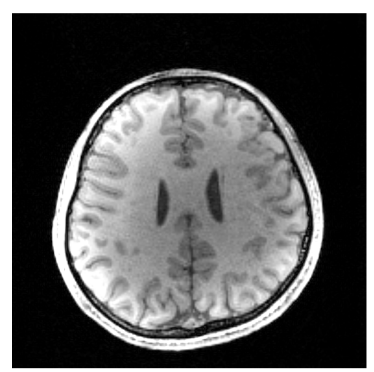

map \#1

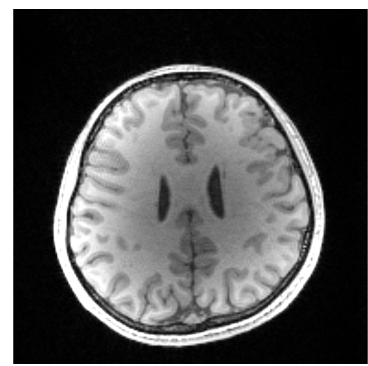

2 maps

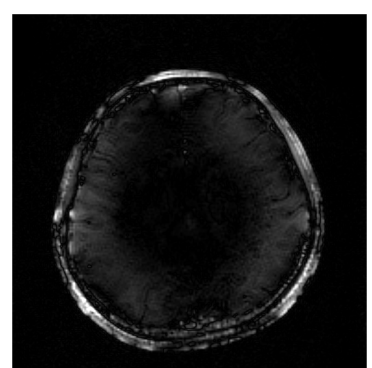

map \#2

VCC

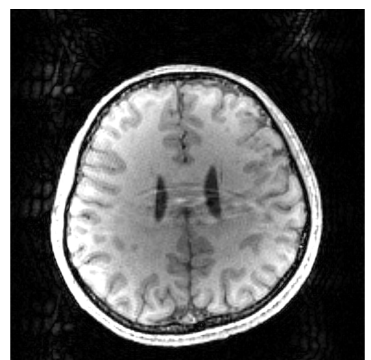

1 map

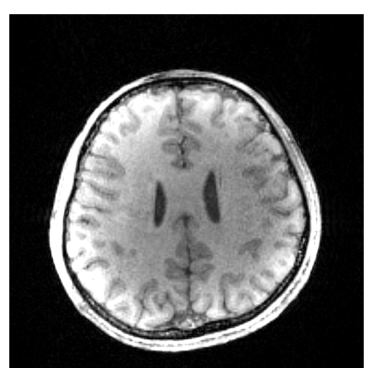

map \#1

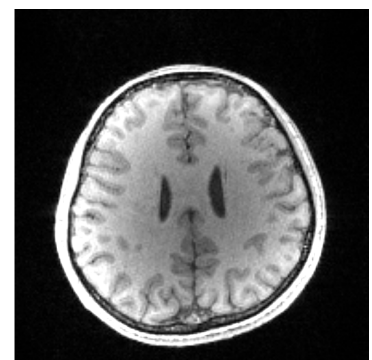

2 maps

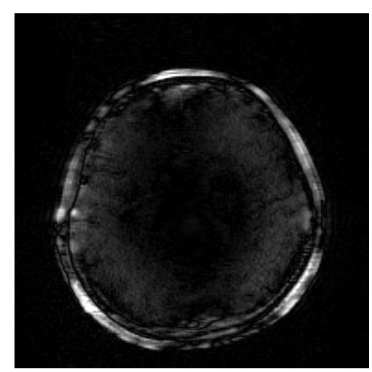

map \#2

PF-VCC

Figure 4.6: ENLIVE reconstructions allowing one and two sets of maps of data extended with virtual conjugate coils (VCC) and such data with a partial $\mathrm{k}$-space (PF-VCC). The virtual-conjugate coils impose a real-value constraint onto the data. High-frequency phase close to the skull violates this constraint, leading to artifacts in reconstructions using a single set of maps. By allowing two sets of maps, these regions with high-frequency phase variation are separated into the second image, allowing almost artifact-free reconstruction. 


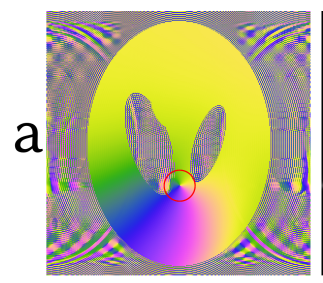

1 map phase

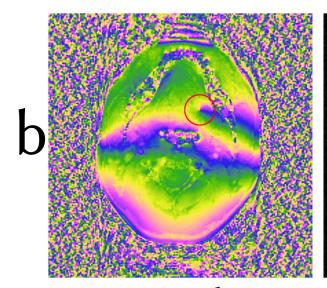

1 map phase

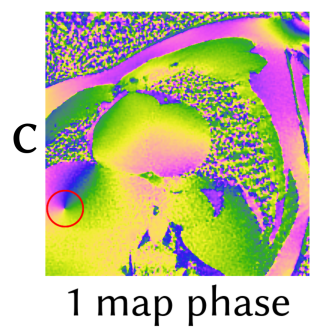

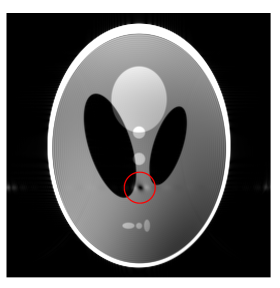

1 map

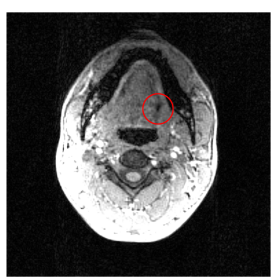

1 map

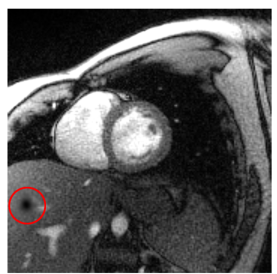

1 map

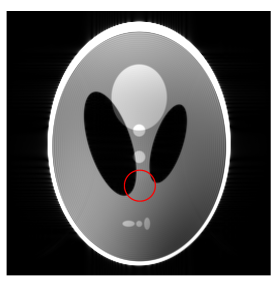

2 maps

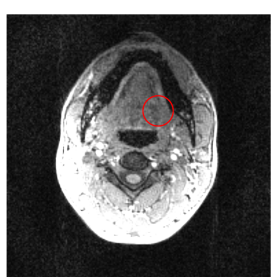

2 maps

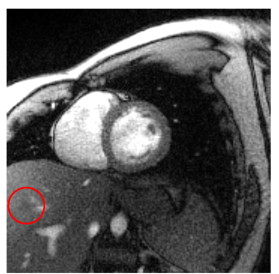

2 maps

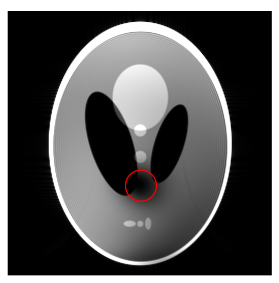

map \# 1

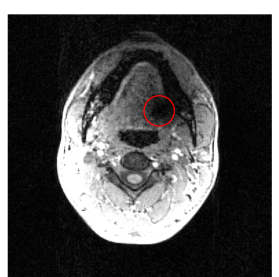

map \#1

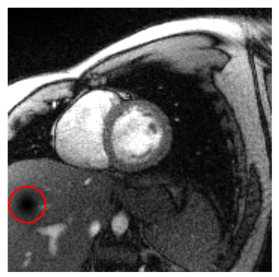

map \#1

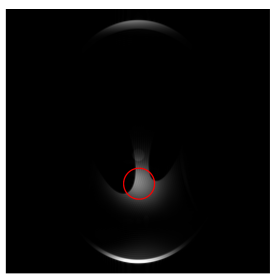

map \#2

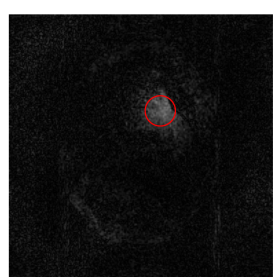

map \#2

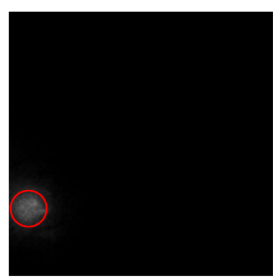

map \#2

Figure 4.7: Phase singularities in (a) a numerical phantom, (b) a transversal slice through the lower jaw and (c) a non-Cartesian short axis-view of the human heart. Each dataset has been reconstructed with ENLIVE allowing one and two sets of maps. The phase singularity in (a) was produced by providing an initial guess containing a singularity. This singularity, clearly visible in the phase image, leads to artifactual signal loss at the same position in the post-processed magnitude image. As in (a), the phase singularities in (b) and (c) lead to signal loss at the corresponding positions in the magnitude images. By allowing two sets of maps, ENLIVE can resolve this artifact by using the second set of sensitivities around the phase singularity, thereby providing an artifact-free combined image. 

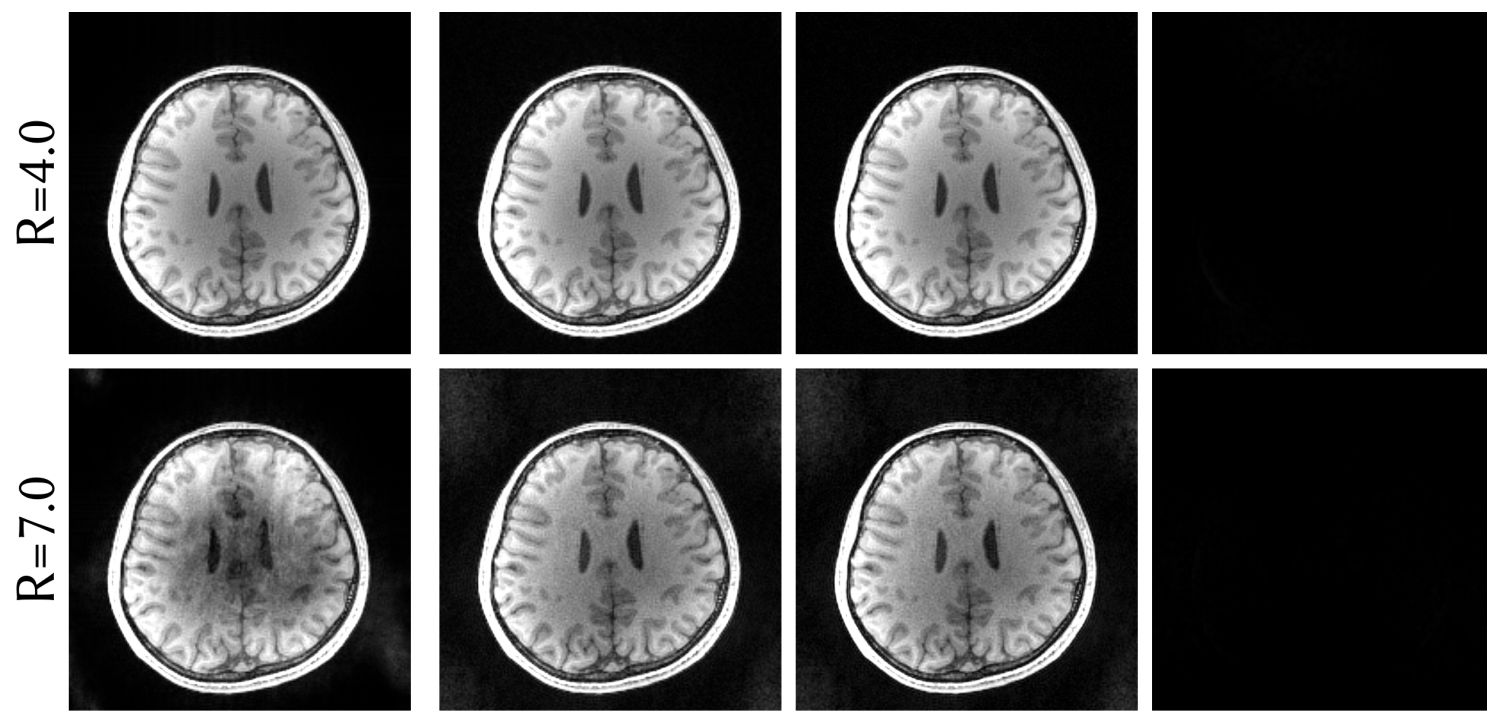

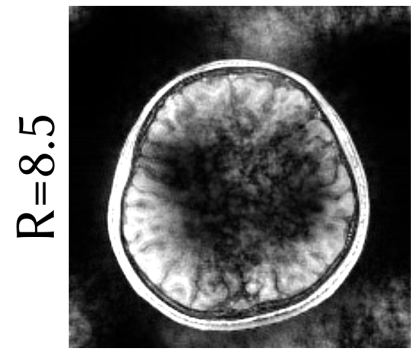

SAKE

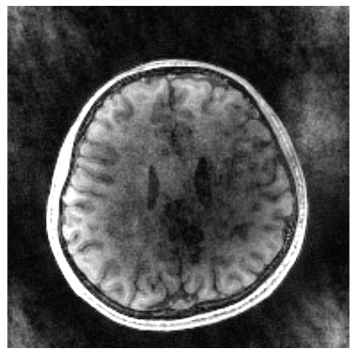

ENLIVE

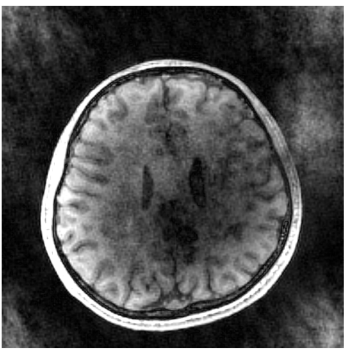

map \#1

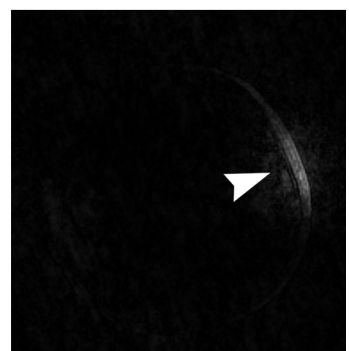

map \#2

Figure 4.8: Variable-density Poisson-disc undersampled data with varying undersampling factors reconstructed with ENLIVE allowing two sets of maps and with SAKE. The same slice as in Fig. 4.6 is used. Since this is a calibrationless parallel imaging reconstruction without additional constraints and without model violations, a single set of maps is sufficient. For undersampling factors up to $\mathrm{R}=7.0$, ENLIVE therefore leaves the second allowed set empty, which causes the combined image to be essentially identical to the first set image. For an undersampling factor of $\mathrm{R}=8.5$, the ENLIVE reconstruction becomes very noisy and some image features start appearing the second map (indicated by an arrow). For $\mathrm{R}=4.0$, SAKE too, provides artifact-free reconstruction. With higher undersampling factors artifacts appear in the images. 

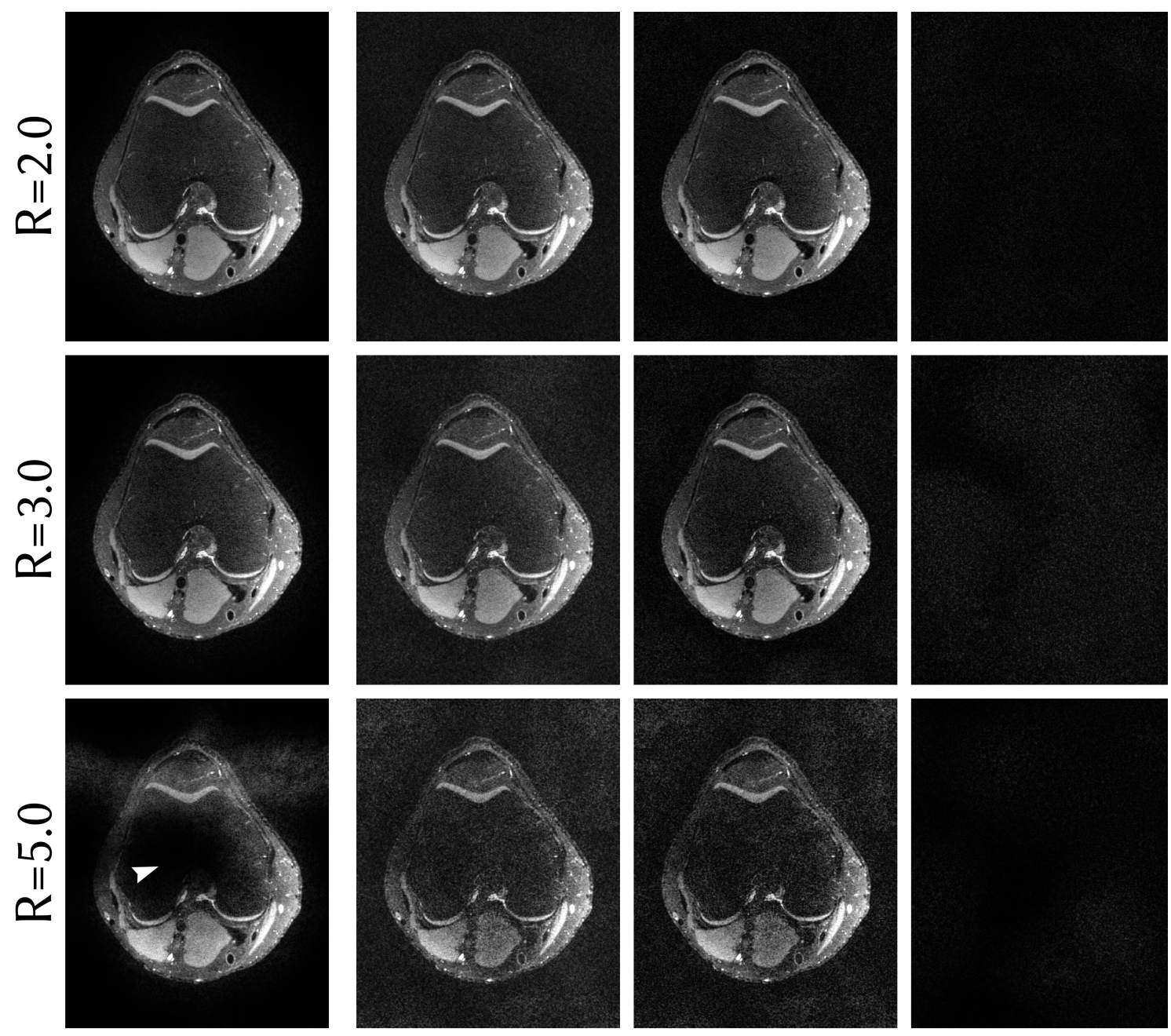

SAKE

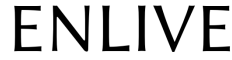

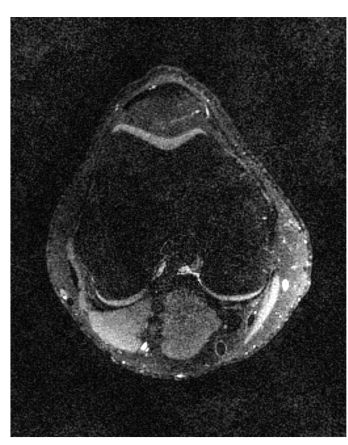

map \#1

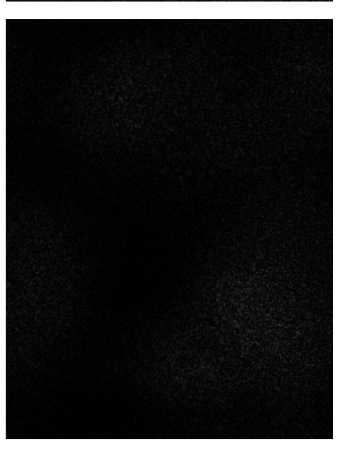

map \#2

Figure 4.9: Variable-density Poisson-disc undersampled data of a human knee with varying undersampling factors reconstructed with ENLIVE allowing two sets of maps and with SAKE This, too, is a dataset without model violations. The second ENLIVE is therefore close to zero. Up to $\mathrm{R}=3.0$, both SAKE and ENLIVE provide artifact free reconstruction. For R=5.0, ENLIVE provides a reconstruction with high noise. SAKE, however, produces a large signal void in the image center (indicated by an arrow). 

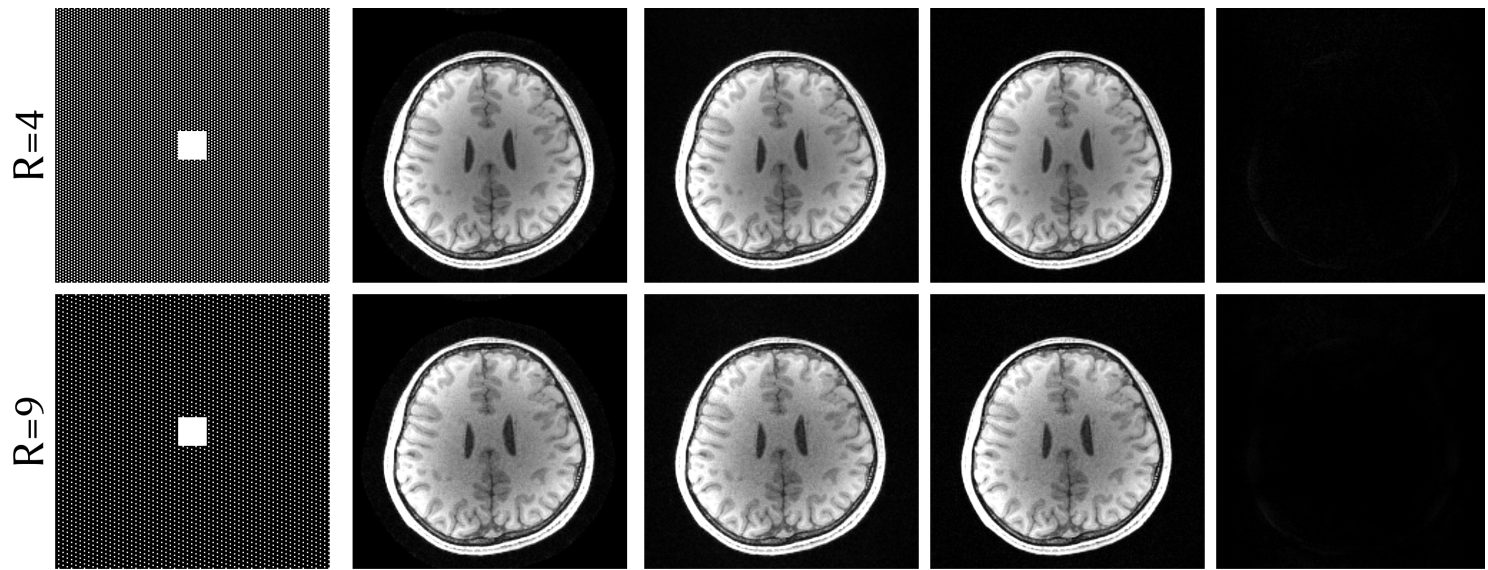

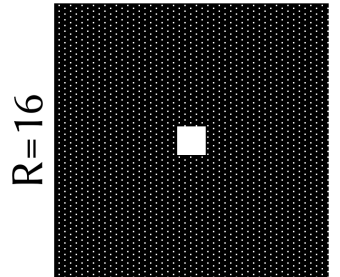

pattern

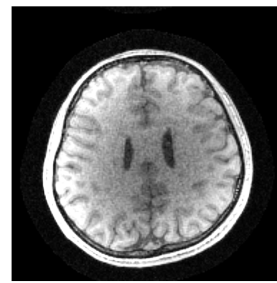

ESPIRiT

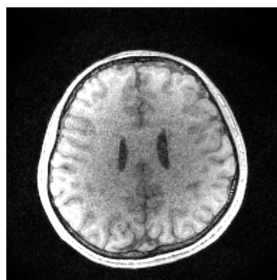

ENLIVE

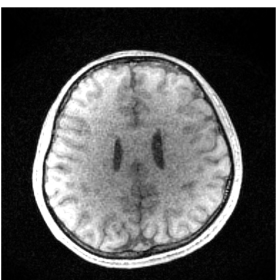

map \#1

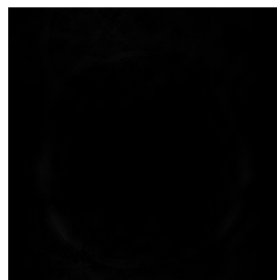

map \#2

Figure 4.10: Comparison of ENLIVE reconstruction using 1 and 2 maps and ESPIRiT reconstruction using 2 maps of the same dataset as in Figure 4.6 undersampled with Cartesian CAIPIRINHA patterns with differing undersampling factors. Using two maps, ENLIVE and ESPIRiT reconstructions show comparable quality. Even though noise is increasing with higher undersampling, the second map remains close to zero. We conjecture that the adequate calibration region inhibits undersampling artifacts and ensures that no signal appears in the second map, in contrast to Figure 4.8 
aliasing artifacts from all pixels in the FOV. The parallel imaging reconstruction using a single set of maps can resolve this aliasing only for pixels outside of the regions of model violation. Since these edge regions alias to the image center, the artifact appears there. Both ENLIVE and ESPIRiT reconstructions allowing multiple sets of maps (Figures 4.1 and 4.2 a) can resolve the aliasing everywhere. For ENLIVE the coil profiles (Figure 4.3) of the second map are sensitive in these regions. For ENLIVE using more than 2 sets of maps, the third and fourth map are close to zero (Figure $4.2 \mathrm{~b}$ ). Since no thresholding is used, they cannot be exactly zero. As is common in parallel imaging, tuning of the regularization is necessary for successful reconstruction: Figure 4.4 shows that using too high regularization (too few Newton steps) does not eliminate the central infolding artifact, while too low regularization (too many Newton steps) leads to high-frequency artifacts. Added noise degrades image quality, especially in the case of too low regularization, but does not change the appearance of the infolding artifact. Additionally, Figure 4.5 shows that the reconstruction is not sensitive to specific choices for the parameters $a$ and $b$ of the coil weighting matrix $\boldsymbol{W}$.

\section{Phase-constrained Imaging}

Next, reconstructions for phase-constrained imaging using virtual-conjugate coils with and without an additional partial-Fourier factor are shown in Figure 4.6 In both cases, reconstruction using only a single set of maps exhibit aliasing artifacts. These are a consequence of the real-value constraint imposed by using virtual-conjugate coils together with high-frequency phase variations caused by off-resonance from fat: A single realvalued image cannot account for this high-frequency phase, therefore the aliasing cannot be resolved. Relaxing the reconstruction by allowing multiple sets of maps resolves this problem, since the second set of maps can now account for this high-frequency phase variation.

\section{Phase Singularities}

Figure $4 \cdot 7$ a shows a phantom example where the initial guess has been intentionally chosen to induce a phase singularity in the reconstruction. The phase singularity leads to signal loss using a single set of maps. Using ENLIVE allowing multiple sets of maps, the affected region can be resolved in the second map. By combining the images, a single image without signal loss can be recovered. This situation can also occur in practice. Figure $4.7 \mathrm{P}$ shows a slice through the throat with large phase variations, while Figure $4.7 \mathrm{~F}$ shows a short-axis view of the human heart acquired with radial FLASH. Using ENLIVE 
allowing multiple sets of maps, it is possible to reconstruct artifact-free images.

\section{Low-rank Property}

Figure 4.8 and Figure 4.9 show calibrationless variable-density Poisson-disc undersampled reconstructions with differing undersampling factors comparing ENLIVE to SAKE In Figure 4.8. both ENLIVE and SAKE provide artifact-free reconstruction for moderate undersampling up to $R=4.0$. At $R=7.0$, SAKE shows artifacts while ENLIVE is artifact free. For these undersampling factors, the second ENLIVE set image is close to zero, while the first set contains the image. For $R=8.5$, both ENLIVE and SAKE show strong artifacts. Additionally, the second ENLIVE map shows some image features. Reconstruction time for $R=4.0$ for this dataset using a single core of an Intel Core i5-459o CPU was $22 \mathrm{~s}$ using ENLIVE and $6.3 \mathrm{~h}$ using SAKE In Figure 4.9 ENLIVE and SAKE provide artifact-free reconstruction up to $R=3.0$. At $R=5.0$, ENLIVE reconstruction is noisy while SAKE shows a large signal void. Reconstruction time for $R=2.0$ for this dataset using a single core was18.6 s using ENLIVE and 41.5 min using SAKE

Figure 4.10 shows Cartesian ENLIVE reconstructions of data undersampled using CAIPIRINHA patterns with different undersampling factors. As a reference, the corresponding patterns are shown in the first column. For all undersampling factors, the second map image is close to zero wile the first map contains the entire image. With increasing undersampling, high noise starts to appear in the first map and the combined image. Still, no undersampling artifacts appear even at $R=16$. Furthermore, even at this high undersampling, no image features appear in the second map, in contrast to the result in Figure 4.8 . We conjecture that the adequate calibration region in this datasets prevents that artifact.

\subsubsection{Discussion}

This work introduces ENLIVE a nonlinear reconstruction method for parallel imaging using a relaxed forward model. Using the IRGNM, ENLIVE simultaneously estimates multiple sets of images and coil sensitivity profiles, extending NLINV by ESPIRiT's approach of using multiple sets of maps. The resulting bi-linear problem with $\ell_{2}$-regularization can be related to a lifted linear formulation using nuclear norm regularization, which promotes low-rank solutions. From this, it becomes apparent that the method, while employing a different parametrization, is similar to SAKE and P-LORAKS [40, 41], which are based on structured low-rank matrix completion in k-space, and to CLEAR [42], which locally promotes low-rankness in the image domain. Although the low-rankness 
of the matrix considered in the k-space methods is also caused by the fact that the signal lives in a sub-space spanned by the coil sensitivities [15, 39], it is constructed from many shifted copies of the signal in k-space. This leads to a huge linear reconstruction problem with a rank constraint. In contrast, CLEAR uses block-wise reconstruction in the image domain, which is more similar to ENLIVE but still requires a large number of small SVDs. A similar concept has been used to implement other low-rank methods. For example, building on top of the work on object modeling introduced in [19], several approaches using annihilating filters have recently been proposed for combining parallel imaging with compressed sensing [51-54]. The existence of annihilating filters implies in turn the existence of a weighted low-rank Hankel matrix which can be constructed from the $\mathrm{k}$-space samples. These methods then recover missing samples by structured low-rank matrix completion. In ENLIVE the convex matrix completion problem has been replaced by a much smaller bi-linear problem with simple quadratic penalties [48, 49]. In some sense, this is similar to the idea of transforming linear problems with $\ell_{1}$-regularization into quadratic problems with $\ell_{2}$-regularization [55].

Low-rank approaches have also been proposed for dynamic imaging. One method for blind compressed sensing [56] estimates both the time series of images as well as a dictionary which sparsifies that series. Haldar and Liang [57] introduce a method which uses partial separability of the signal into functions describing its k-space and its time dependence. Both of these approaches exploit the low rank of the time-dependent signal. While structurally similar, Haldar and Liang [57] use an explicitly rank-constraint formulation while Lingala et al. [56] use an $\ell_{1}$-norm to induce sparsity. In contrast, ENLIVE]s $\ell_{2}$-regularization achieves low-rankness even below its constraint on the maximum rank through the equivalence to a formulation with regularization of the nuclear norm outlined in the Theory, which forms the core of the proposed method.

ENLIVE can also be related to a previous extension of NLINV proposed for separation of chemical species 58,59]. This method is based on the idea that the signal is a superposition of different images shifted in the spatial domain according to the chemical shift. As also shown for ESPIRiT, the sensitivities for the shifted signals from different species also appear to be shifted. They therefore violate the simple SENSE model with a single set of of maps and, consequently, cause the appearance of a second set of maps. The previously proposed extension to NLINV can be understood as a version of ENLIVE with the additional constraint that different sets of sensitivities are shifted versions of each other.

As shown in this work, small FOV and phase-constrained reconstructions using a single set of maps show artifacts whenever there are inconsistencies which cannot be explained 
using the simple model, while ENLIVE allowing two sets of maps enables artifact-free reconstruction in all evaluated cases. When using correct regularization, added noise does not impede artifact removal either. In cases where reconstruction with a single set of maps is already free from artifacts, ENLIVE automatically only uses a single set. In general, though, the maximum number of ENLIVE maps must be specified manually. This is similar to ESPIRiT where, while theoretically the correct number of maps can automatically be estimated as the multiplicity of the eigenvalue 1 , in practice a maximum number of maps is set in advance to enable efficient computation of the eigenvector maps by power iteration. However, an extension to ENLIVE to automatically adapt the number of maps during the iteration is also conceivable.

As the distribution of the phase between image and coil sensitivities cannot be determined from the data alone without additional prior knowledge, choosing a good phase is a common problem when calibrating sensitivities 60 61]. This fundamental problem affects different algorithms in different ways. In Walsh's method [62] or ESPIRiT a pixel-wise phase across channels simply remains undefined and has to be aligned to a reference. If the reference is not ideal, phase singularities may occur. Phase singularities imply a non-smooth phase which then reduces sparsity in compressed sensing, preventing an efficient and compact representation of the sensitivities in the Fourier domain [63], or causing problems in post-processing. For example, as Li et al. [64 have shown, phase singularities can appear as artifactual microhemorrhage in susceptibility weighted imaging. NLINV and ENLIVE guarantee smooth sensitivities, but this then traps the algorithm in a local minimum and creates a hole instead [65]. For ENLIVE, the use of a second set of maps may still avoid signal loss in the reconstruction.

Even though local minima are a general concern with nonlinear methods, in our experience, the only practically relevant examples are the phase singularities. There, although the ENLIVE reconstruction is not optimal, use of a second map may mitigate the resulting artifact.

Compared to ESPIRiT, ENLIVE is more flexible since it has fewer prerequisites for its use, e.g. no calibration region is necessary. However, in the case of an undersampled Cartesian acquisition with calibration region, ESPIRiT is still to be preferred in most cases because of its speed. Only when faced with a very large number of channels might ESPIRiT lead to longer reconstruction times due to the unfavorable scaling of its SVD with the number of channels.

In summary, ENLIVE combines different advantages of NLINV ESPIRiT, and SAKE. As NLINV and SAKE it utilizes all available data, can be directly applied to non-Cartesian data, and does not require a calibration region. As ESPIRiT and SAKE, it is not limited to the 
SENSE model but automatically adapts to certain inconsistencies in the data. As ESPIRiT and NLINV] it is computationally efficient and makes use of an explicit image-domain representation during reconstruction which facilitates the use of advanced regularization terms.

\subsubsection{Conclusion}

In this work we propose ENLIVE a nonlinear method for parallel imaging which seeks to combine the robustness of ESPIRiT with the flexibility of NLINV ENLIVE can be related to a lifted formulation of blind multi-channel deconvolution with nuclear norm regularization, which show that it belongs to the class of calibrationless parallel imaging methods based on structured low-rank matrix completion. In imaging settings involving limited FOV, phase constraints, and phase singularities, it has been shown to provide artifact-free reconstruction with quality comparable to state-of-the-art methods.

\subsubsection{Methods}

The proposed method was implemented in the Berkeley Advanced Reconstruction Toolbox (BART) [37] and all other reconstructions were performed using BART as well. Processlevel parallelization was achieved using GNU parallel [66]. To facilitate the reproducibility of our research, data and source code used to generate the results of this paper can be downloaded from https://github.com/mrirecon/enlive

To test its robustness in case of inconsistencies, ENLIVE was applied in several different experimental settings: We selected examples for imaging with an FOV smaller than the extent of the object, phase-constrained imaging, and phase singularities. In all cases, reconstructions using ENLIVE were performed using one, i.e. NLINV or two sets of maps with initial regularization set to $\alpha_{0}=1$. If not stated otherwise, 11 Newton steps and $q=1 / 2$ were used for the IRGNM These parameters, as well as the parameters for the other methods, were chosen according to best visual appearance.

All volunteer imaging for this study was performed with their prior informed written consent, in accordance with the relevant guidelines and regulations, and with the approval of the ethics committee of the University Medical Center Göttingen.

In an example without inconsistencies we tested whether ENLIVE produces results with only one set of maps. Additional examples show ENLIVE s performance under high undersampling and in non-Cartesian imaging. 


\section{Limited FOV}

We applied ENLIVE to the same dataset used in [15]. This is a retrospectively 2 -fold undersampled $2 \mathrm{D}$ spin-echo dataset $\left(\mathrm{TR} / \mathrm{TE}=550 / 14 \mathrm{~ms}, \mathrm{FA}=90^{\circ}, \mathrm{BW}=19 \mathrm{kHz}, \mathrm{ma}-\right.$ trix size: $320 \times 168$, slice thickness: $3 \mathrm{~mm}, 24 \times 24$ calibration region) with an FOV of $200 \times 150 \mathrm{~mm}^{2}$, acquired at $1.5 \mathrm{~T}$ using an 8 -channel head coil. The dataset was zeropadded in k-space to produce square image space pixels. This FOV is smaller than the head of the subject in the lateral direction which leads to artifacts in a traditional SENSE reconstruction. These data were reconstructed with ENLIVE using one or two sets of maps and compared to ESPIRiT using one or two sets of maps. To investigate the effect of additional sets of maps, the data were additionally reconstructed using 1, 2, 3, and 4 sets of maps. For ENLIVE $q=2 / 3$ was used. To investigate the sensitivity to noise and to regularization, an additional reconstruction using 13, 16, 19, 22 and 25 Newton steps and added Gaussian white noise with noise levels of $0 \%, 0.1 \%, 1 \%, 2.5 \%$ and $5 \%$ was performed. The noise level here is the standard deviation of the added noise as percent of the magnitude of the DC component. From this, 19 Newton steps was determined as the optimum and used for reconstruction. For ESPIRiT a kernel size of $6 \times 6$ and a threshold of 0.001 was used.

\section{Phase-constrained Imaging}

Phase-constrained parallel imaging [67] with virtual conjugate coils [68] is equivalent to an explicit phase constraint in SENSE but more robust in GRAPPA and ESPIRiT due to their ability to adapt to inconsistencies 44, 69]. To assess ENLIVE s performance in phase-constrained imaging settings with virtual conjugate coils, we applied it to the same dataset used in [44]. This is a single slice in readout direction of a retrospectively 3 -fold undersampled $3 \mathrm{D}$ FLASH dataset $(\mathrm{TR} / \mathrm{TE}=11 / 4.9 \mathrm{~ms})$ acquired at $3 \mathrm{~T}$ using a 32 -channel head coil. $24 \times 24$ auto-calibration lines were used. Additionally, a partial Fourier factor of $5 / 8$ was applied to the data and evaluated separately.

\section{Phase Singularities}

Similar to other algorithms [60,61, 65] phase singularities can appear in coil sensitivity profiles with ENLIVE As ENLIVE enforces smooth coil sensitivity profiles, this leads to an artifactual hole in the sensitivities around the singularity. To demonstrate this effect, we synthetically constructed an example using BART to generate 6-channel k-space data (matrix size: $256 \times 256$ ) of the numerical Shepp-Logan phantom. To get ENLIVE trapped in 
a local minimum with a phase singularity, we provided an initial guess already containing a phase singularity. In regions with rapid phase variation, such phase singularities can also appear in ENLIVE reconstructions of in-vivo data. A transversal slice through the throat containing such a phase singularity was selected from the same dataset used for phase-constrained imaging.

To further show that ENLIVE can be applied directly to non-Cartesian data, we reconstructed selected data containing a phase singularity from a real-time FLASH [30] acquisition using a 30 channel thorax coil of a short-axis view through the heart of a volunteer with no known illnesses $\left(\mathrm{TR} / \mathrm{TE}=2.22 / 1.32 \mathrm{~ms}, \mathrm{FA}=10^{\circ}\right.$, matrix size: $160 \times 160$, FOV $=256 \times 256 \mathrm{~mm}^{2}$, slice thickness: $6 \mathrm{~mm}$, field strength: $3.0 \mathrm{~T}$ ). Five consecutive frames during diastole, comprising 65 radial spokes, were selected, corrected for gradient delays [70], regridded to a 1.5 times finer grid and subsequently reconstructed with ENLIVE using 1 and 2 maps. For this dataset, $q=2 / 3$ and 17 iterations of the IRGNM were used.

\section{Low-rank Property}

In order to show that ENLIVE automatically uses only the required number of sets of maps, we retrospectively undersampled the same $3 \mathrm{D}$ dataset used for phase-constrained imaging using variable-density Poisson-disc sampling [36] with undersampling factors of $R=4.0,7.0,8.5$ and without a calibration region, and then extracted the same slice in readout direction. As a comparison, these data were also reconstructed using SAKE with 50 iterations and a relative size of the signal subspace of 0.05 .

Additionally, we applied SAKE and ENLIVE to a 3D fast spin-echo acquisition [71] of a human knee $\left(\mathrm{TR} / \mathrm{TE}=1550 / 25 \mathrm{~ms}, \mathrm{FA}=90^{\circ}\right.$, echo train length: 40 , matrix size: $320 \times 256$, FOV $=160 \times 153.6 \mathrm{~mm}^{2}$, field strength: $3.0 \mathrm{~T}$ ) from mridata.org 72. This dataset was also undersampled using variable-density Poisson-disc sampling with undersampling factors of $R=2,3,5$ and a single slice in readout direction was extracted. These data were then reconstructed using ENLIVE with 1 and 2 maps and with SAKE with 50 iterations and a relative size of the signal subspace of 0.125 .

To evaluate ENLIVE in settings with high acceleration factors, we undersampled the $3 \mathrm{D}$ dataset used for phase-constrained imaging using Cartesian CAIPIRINHA [73] patterns with undersampling factors of $R=4,9,16$ with a $24 \times 24$ calibration region. These data were then reconstructed with ENLIVE using 2 maps with $q=1 / 3$ and 8 iterations of the IRGNM 


\subsubsection{Appendix}

\section{Operator $\mathcal{A}$}

Here, we show the layout of $\boldsymbol{u}, \boldsymbol{v}$, and $\boldsymbol{u} \boldsymbol{v}^{T}$ as well as the action of $\mathcal{A}$ using an image of size $N_{I}:=n_{x} \cdot n_{y} \cdot n_{z}$ and $N_{C}$ coils. Then, the vector $\boldsymbol{u} \equiv \boldsymbol{m} \in C^{N_{I}}$ is defined as

$$
\boldsymbol{u}^{T}=\left(\begin{array}{lll}
m_{1} & \ldots & m_{N_{I}}
\end{array}\right)
$$

and the vector $\boldsymbol{v} \in C^{N_{C} \cdot N_{I}}$ of stacked, weighted coil sensitivity profiles $\hat{\boldsymbol{c}}_{j}$ as

$$
\boldsymbol{v}^{T}=\left(\begin{array}{llllll}
\hat{c}_{1,1} & \ldots & \hat{c}_{N_{I}, 1} & \hat{c}_{2,2} & \ldots & \hat{c}_{N_{I}, N_{C}}
\end{array}\right)
$$

where $\hat{c}_{i j}$ is the $i$ th pixel of the $j$ th weighted coil profile. Therefore, $u \boldsymbol{v}^{T} \in C^{N_{I} \times N_{C} \cdot N_{I}}$ is

$$
\boldsymbol{u} \boldsymbol{v}^{T}=\left(\begin{array}{cccccccc}
m_{1} \hat{c}_{1,1} & m_{1} \hat{c}_{2,1} & \ldots & m_{1} \hat{c}_{N_{I}, 1} & m_{1} \hat{c}_{1,2} & m_{1} \hat{c}_{2,2} & \ldots & m_{1} \hat{c}_{N_{I}, N_{C}} \\
m_{2} \hat{c}_{1,1} & m_{2} \hat{c}_{2,1} & \ldots & m_{2} \hat{c}_{N_{I}, 1} & m_{2} \hat{c}_{1,2} & m_{2} \hat{c}_{2,2} & \ldots & m_{2} \hat{c}_{N_{I}, N_{C}} \\
\vdots & \vdots & \ddots & \vdots & \vdots & \vdots & & \vdots \\
m_{N_{I}} \hat{c}_{1,1} & m_{N_{I}} \hat{c}_{2,1} & \ldots & m_{N_{I}} \hat{c}_{N_{I}, 1} & m_{N_{I}} \hat{c}_{1,2} & m_{N_{I}} \hat{c}_{2,2} & \ldots & m_{N_{I}} \hat{c}_{N_{I}, N_{C}}
\end{array}\right)
$$

Applying the inverse of the weighting matrix $W$ yields

$$
\boldsymbol{u} \boldsymbol{v}^{T} \boldsymbol{W}^{-1}=\left(\begin{array}{cccccccc}
\boldsymbol{m}_{1} \boldsymbol{c}_{1,1} & m_{1} c_{2,1} & \ldots & m_{1} c_{N_{I}, 1} & \boldsymbol{m}_{1} \boldsymbol{c}_{1,2} & m_{1} c_{2,2} & \ldots & m_{1} c_{N_{I}, N_{C}} \\
m_{2} c_{1,1} & \boldsymbol{m}_{2} \boldsymbol{c}_{2,1} & \ldots & m_{2} c_{N_{I}, 1} & m_{2} c_{1,2} & \boldsymbol{m}_{2} \boldsymbol{c}_{2,2} & \ldots & m_{2} c_{N_{I}, N_{C}} \\
\vdots & \vdots & \ddots & \vdots & \vdots & \vdots & & \vdots \\
m_{N_{I}} c_{1,1} & m_{N_{I}} c_{2,1} & \ldots & \boldsymbol{m}_{N_{I}} \boldsymbol{c}_{N_{I}, 1} & m_{N_{I}} c_{1,2} & m_{N_{I}} c_{2,2} & \ldots & \boldsymbol{m}_{N_{I}} \boldsymbol{c}_{N_{I}, N_{C}}
\end{array}\right)
$$

The diagonals containing products of image pixels with corresponding coil profile pixels are highlighted in bold. The action of the operator $\mathcal{A}$ is to select these highlighted entries of $\boldsymbol{u} \boldsymbol{v}^{T} \boldsymbol{W}^{-1}$, apply a two or three dimensional Fourier transform to each coil image and finally apply a mask $\mathcal{P}$ projecting onto the acquired pattern.

\section{Equivalence of formulations}

In the following we will show that the lifted rank- $k$ problem in Equation 4.4 corresponds to the ENLIVE formulation in Equation 4.2. Using the linearity of the operators, the 
following holds:

$$
\begin{aligned}
\mathcal{A}\left\{\boldsymbol{U} \boldsymbol{V}^{T}\right\} & =\mathcal{A}\left\{\sum_{i=1}^{k} \boldsymbol{u}_{i} \boldsymbol{v}_{i}^{T}\right\} \\
& =\sum_{i=1}^{k} \mathcal{A}\left\{\boldsymbol{u}_{i} \boldsymbol{v}_{i}^{T}\right\} \\
& =\sum_{i=1}^{k}\left(P \mathcal{F}\left\{\boldsymbol{c}_{j}^{i} \odot \boldsymbol{m}^{i}\right\}\right)_{j=1 \ldots N_{c}} \\
& =\left(P \mathcal{F}\left\{\sum_{i=1}^{k} \boldsymbol{c}_{j}^{i} \odot \boldsymbol{m}^{i}\right\}\right)_{j=1 \ldots N_{c}}
\end{aligned}
$$

Here, we make use of the definition of the operator $\mathcal{A}$ :

$$
\mathcal{A}\left\{\boldsymbol{u}_{i} \boldsymbol{v}_{i}^{T}\right\}:=\left(P \mathcal{F}\left\{\boldsymbol{c}_{j}^{i} \odot \boldsymbol{m}^{i}\right\}\right)_{j=1 \ldots N_{c}}
$$




\subsection{Pre-Calibration of Coil Sensitivities using ENLIVE}

Generally, current MRI is a two step process: in a first step, coil sensitivity profiles are calculated, which are used in the second step, the actual reconstruction. This precalculation converts the generally non-linear MRI problem into a linear inverse problem, so the resulting reconstruction is also a linear parallel imaging reconstruction or a combined parallel imaging and compressed sensing (PICS) reconstruction with a linear model. Splitting the reconstruction into two steps reduces the problem complexity and thereby improves reconstruction speeds.

While several methods for coil sensitivity calculation exist, ESPIRiT [15] has in recent years emerged as a de-facto standard in MRI research applications. It is fundamentally based on a singular value decomposition (SVD) of an image space operator. Since the SVD has an unfavorable scaling with the size of the matrix 1 and the ESPIRiT operator in question scales with the number of coil sensitivities that need to be determined, ESPIRiT, too, scales unfavorably with the number of coils.

Another disadvantage of current methods for coil sensitivity pre-calibration is that they need a relatively large calibration region in the k-space center to obtain high quality coil sensitivity profiles. Iterative SVD-based methods such as SAKE [39] can be applied to partially undersampled calibration data, but are even more computationally demanding, as they use the SVD inside of an iterative algorithm. For this reason, the use of non-linear methods such as NLINV as a calibration step for linear reconstruction methods is an attractive alternative: For example Roeloffs et al. [74] and Wang et al. [75] used it to pre-calculate coil sensitivity profiles for their model-based reconstructions. Still, NLINV lacks the robustness of ESPIRiT, which can make use of multiple maps.

To explore the use of ENLIVE to provide accurate coil sensitivity profiles, I applied ENLIVE and ESPIRiT to the central region of a $3 \mathrm{D}$ magnetization-prepared rapid gradientecho (MPRAGE) acquisition acquired with a 64-channel head coil. Additional sequence parameters are $B_{0}: 3 \mathrm{~T}$, TR/TE: $2300 \mathrm{~ms} / 2.27 \mathrm{~ms}$, flip angle: $8^{\circ}$, matrix size: $448 \times 224 \times 224$, resolution: $1 \times 1 \times 1 \mathrm{~mm}^{3}$. This dataset was undersampled in the two phase-encoding directions using Poisson-disc undersampling with a factor of 4 . A central region of size $72 \times 48 \times 48$ was extracted for coil sensitivity calculation, compressed with a singular value decomposition (SVD) to 2 to 64 channels, and then coil sensitivities were calculated using ESPIRiT with 2 maps and by reconstructing this reduced dataset with ENLIVE using 2 maps. The resulting sensitivities were then used in a linear parallel imaging reconstruction. As an example, selected orthogonal slices for 16 channels are shown in

${ }^{1}$ Computing the SVD of an $m \times n$ matrix has a complexity of $O(m n \min (n, m))$ 
Figure 4.11 together with their difference, showing that both ESPIRiT and ENLIVE provide accurate coil sensitivity profiles which enable high-quality reconstruction.

In such settings, ENLIVE can be advantageous because of its speed: to compare ESPIRiT calibration to ENLIVE reconstruction, the time needed for both is shown in Figure 4.12 Even though ESPIRiT is faster for few channels, it scales between quadratically and cubically with the number of channels, while ENLIVE scales linearly. Therefore, ENLIVE is significantly faster for a higher number of channels.

This idea of using ENLIVE to calculate coil sensitivity profiles for later robust linear reconstruction is already used in a study by Rosenzweig et al. [76]. There, ENLIVE]coil sensitivity profiles with 2 maps are used to reconstruct self-gated cardiac MRI data. In the future, ENLIVE might emerge as an alternative to ESPIRiT for coil sensitivity calibration in settings where high-quality and robust coil profiles are necessary, but where full non-linear reconstruction is not needed.

${ }^{2}$ All times given are with 2 threads on an Intel Core i5-4590 processor. 

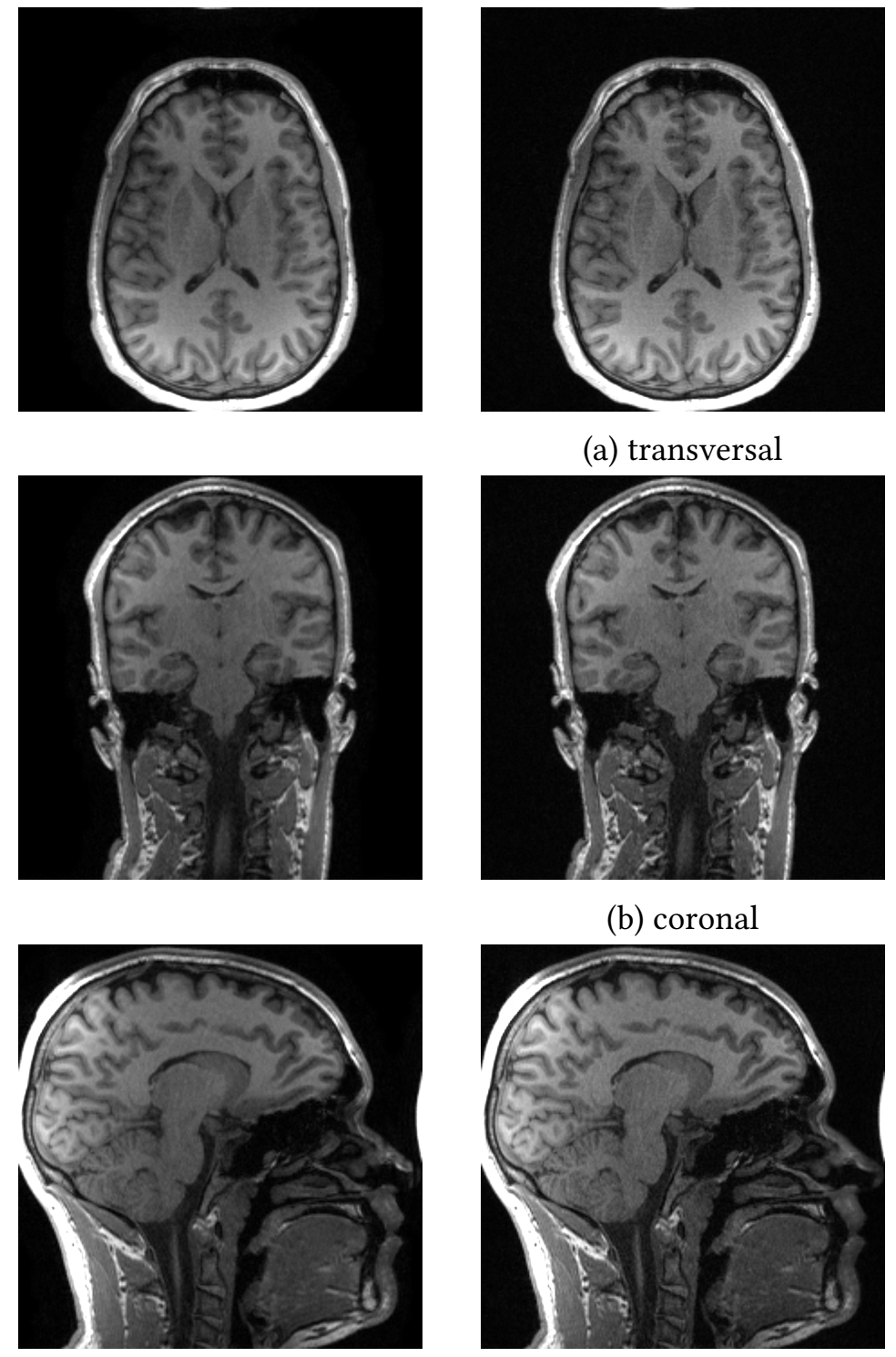

(c) sagittal

(a) transversal

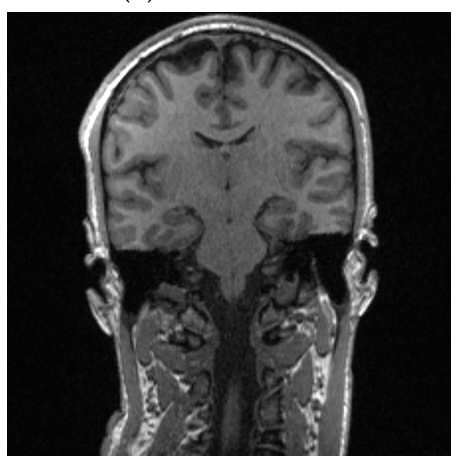

(b) coronal
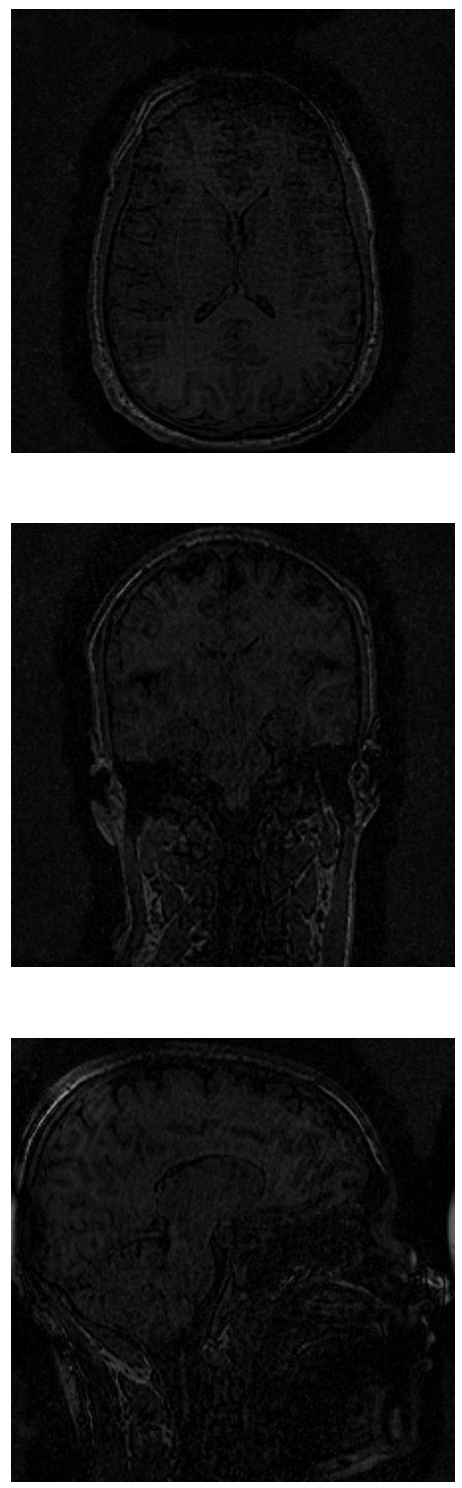

Figure 4.11: Selected orthogonal slices of a linear parallel imaging reconstruction with coil profiles calculated by ESPIRiT (left) and ENLIVE(center) together with their difference (right) scaled by a factor of $\times 2$. For this reconstruction, the original data was compressed to 16 virtual channels using an SVD. Both reconstructions show comparable and high quality, showing that both ESPIRiT and ENLIVE provided accurate coil sensitivity profiles. 

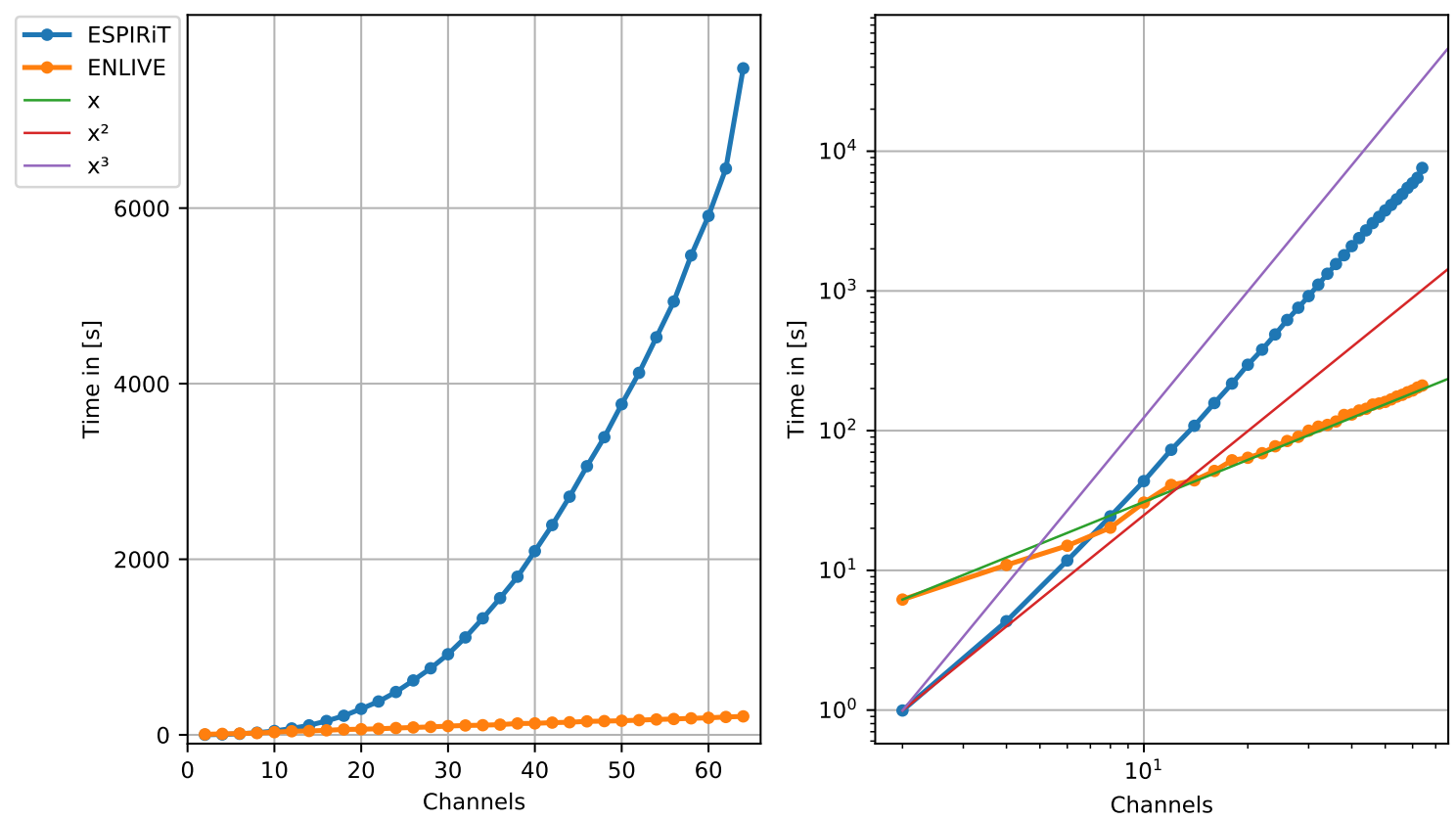

Figure 4.12: Left: Time needed for the calculation of the coil sensitivities using both ENLIVE and ESPIRiT. Right: log-log-plot of the same data, with lines showing linear, quadratic and cubic growth. For easier comparison, the line showing linear growth is scaled to start at the first ENLIVE point, while the lines for quadratic and cubic growth start at the first point for ESPIRiT.

From these results, we can see that ESPIRiT is faster for small numbers of coils $(<8)$, but that ENLIVE scales approximately linearly with the number of coils whereas ESPIRiT scales somewhere between quadratically and cubically. So for larger numbers of coils, ENLIVE]s favorable scaling leads to shorter calibration times. 


\subsection{ENLIVE with Temporal Regularization: Robust Reconstruction for real-time MRI}

Real-time MRI [30, 77] is any method of acquiring and reconstructing MRI images at sufficient speed to resolve dynamic processes such as breathing motion or heart movement. This is desirable since it enables acquisitions of for example cardiac structure and function without assumptions about beat-to-beat similarity, which are necessary for conventional CINE MRI Here, too, regularized non-linear inverse reconstruction (NLINV) has emerged as a successful technique because of its ability to reconstruct non-Cartesian data without pre-calibration. Real-time NLINV] has so far been used for structural [30, 78] and phasecontrast flow [79, 80] imaging of the heart, for MRI-guided biopsy of cardiac tissue in a pig model [81], for the diagnosis of gastroesophageal reflux disease [82] and for fast reconstruction of simultaneous multi-slice data [83]. However, NLINV]can suffer from phase singularities, which manifest as local signal voids (see Figure $4 \cdot 7$ on page 31 ). Therefore, I will investigate if an extension of ENLIVE to real-time data can mitigate these signal voids, analogous to its performance on static images shown in Figure $4 \cdot 7$

This extension can be done in a way resembling the extension of NLINV to real time data. For this, Equation (4.2) can be modified to include regularization with respect to the previous frame:

$$
\underset{\boldsymbol{m}^{i}, \boldsymbol{c}_{j}^{i}}{\arg \min } \sum_{j=1}^{N_{C}}\left\|\boldsymbol{y}_{j}-\mathcal{P} \mathcal{F}\left\{\sum_{i=1}^{k} \boldsymbol{c}_{j}^{i} \odot \boldsymbol{m}^{i}\right\}\right\|_{2}^{2}+\alpha \sum_{i=1}^{k}\left(\sum_{j=1}^{N_{C}}\left\|\boldsymbol{W}\left(\boldsymbol{c}_{j}^{i}-\beta \boldsymbol{c}_{j, 0}^{i}\right)\right\|_{2}^{2}+\left\|\left(\boldsymbol{m}^{i}-\beta \boldsymbol{m}_{0}^{i}\right)\right\|_{2}^{2}\right) .
$$

Here, $\boldsymbol{c}_{j, 0}^{i}$ and $\boldsymbol{m}_{0}^{i}$ are the coil profiles and images of the previous frame used as a reference, weighted by a factor $\beta$ with usually $\beta=0.9$. Additionally, the reconstruction of each frame is initialized with the results of the previous frame to speed up convergence.

Just as Equation 4.2, Equation 4.8) can also be solved with the IRGNM and, when using $k=1$, i.e. a single image and a single set of coil sensitivity profiles, this is identical to real-time NLINV

In order to evaluate real-time ENLIVE, I implemented Equation 4.8 in BART and reconstructed interactiv ${ }^{3}$ real-time MRI data using 1 and 2 maps. I selected a radial FLASH dataset acquired at $3 \mathrm{~T}$ with TR/TE: $2.02 \mathrm{~ms} / 1.3 \mathrm{~ms}$, flip angle: $8^{\circ}, \mathrm{FOV}=256 \times 256 \mathrm{~mm}^{2}$, slice thickness: $8 \mathrm{~mm}$, base resolution: 128 , with 21 uniformly distributed spokes per frame. In this dataset, consisting of a total of 4807 frames over $204 \mathrm{~s}$, the slice was moved

\footnotetext{
${ }^{3}$ This means that the acquired slice can be freely moved during the acquisition
} 
and rotated through a variety of views of the human heart. Selected frames of this reconstruction are shown in Figure 4.13

Image quality of both methods is similar (see their windowed difference in Figure 4.14), however, the reconstruction using a single map show obvious signal voids related to phase singularities (compare Figure 4.7 on 31). A difference to the case of static images is that in real-time NLINV and ENLIVE phase singularities can move between frames. Also, even using two maps, they are not immediately resolved: Figure 4.13(b) clearly shows signal voids even using 2 maps. However, in Figure 4.13(c) ten frames (about $430 \mathrm{~ms}$ ) later, the signal voids are removed using 2 maps. Similarly in a later frame shown in Figure $4.13(\mathrm{~d})$

This late removal might be due to the temporal regularization: even using 2 maps, each frame is initialized and regularized with the previous frame. This limits the deviation to that frame and may slow the removal of the signal void, even though the data fidelity term in Equation (4.8) is certainly smaller without such a void. Additionally, real-time data is usually reconstructed with fewer Newton steps and therefore with higher final regularization. This, too, might explain the time lag in removing signal voids.

Still, in real-time applications, such short delays are a minor issue and real-time ENLIVE can be used for more robust reconstruction. 

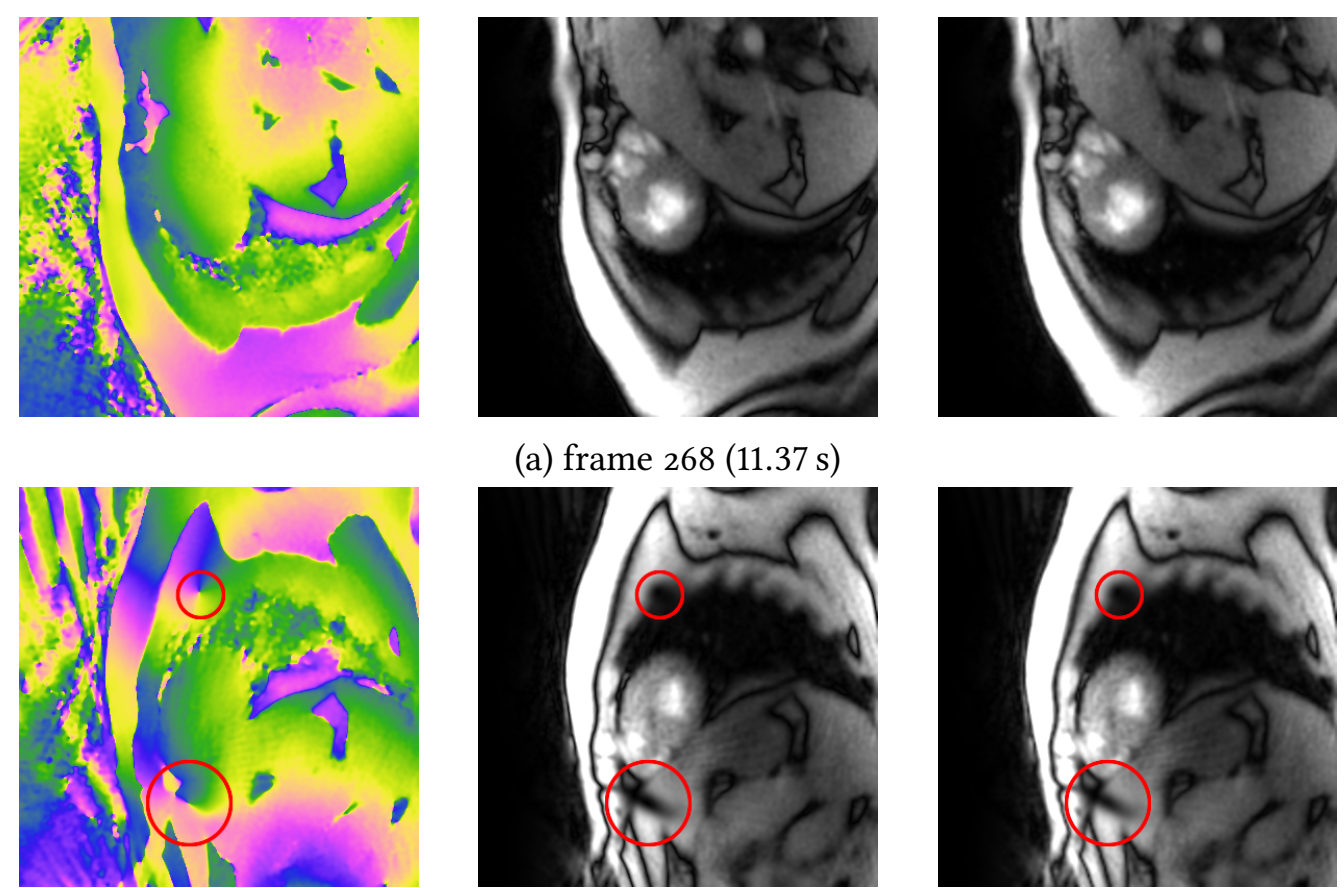

(a) frame $268(11.37 \mathrm{~s})$
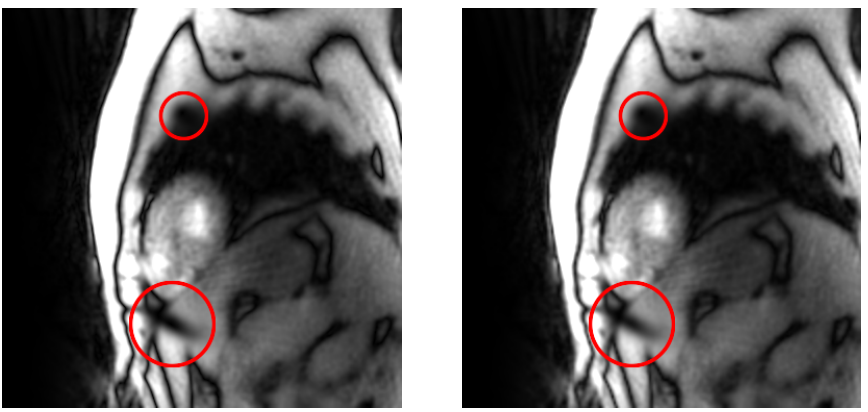

(b) frame $284(12.05 \mathrm{~s})$
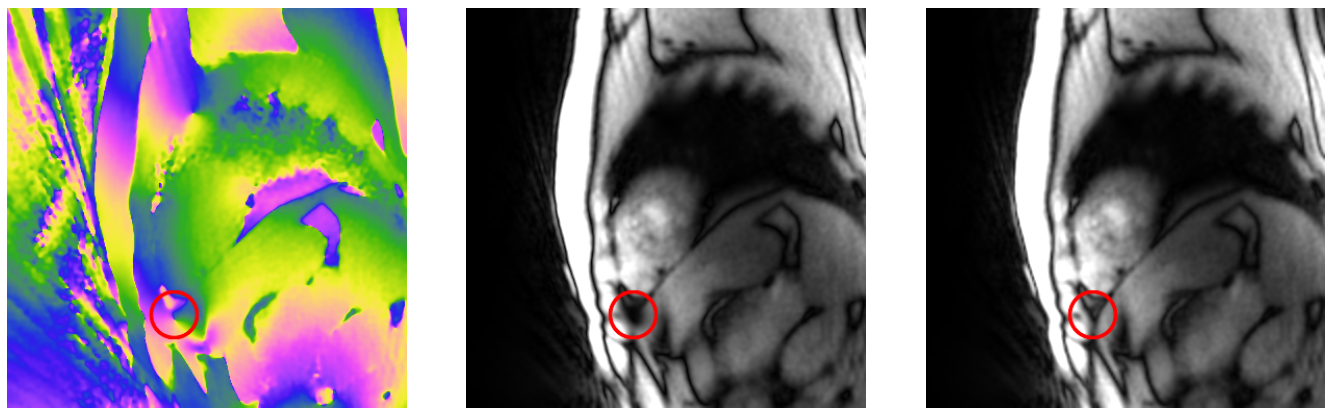

(c) frame $294(12.47 \mathrm{~s})$
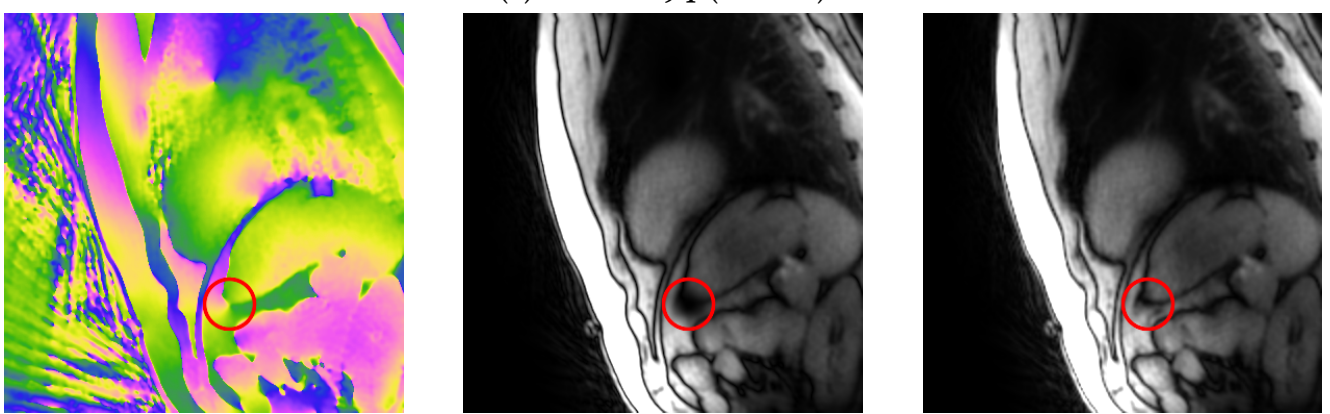

(d) frame $398(16.88 \mathrm{~s})$

Figure 4.13: Reconstruction of selected frames of an interactive real-time acquisition. Left: phase of real-time NLINV ENLIVE with 1 map), center: real-time NLINV, right:ENLIVE with 2 maps. Phase singularities leading to signal voids are highlighted by red circles. As can be seen, using ENLIVE with 2 maps can remove these signal voids, although this can take some frames (see (b) to (c) 10 frames later). The unusual rotation of (a) and (d) is due to the free movement of the slice during acquisition. For better visualization, Figure 4.14 shows the same frames windowed to highlight low-intensity regions. 

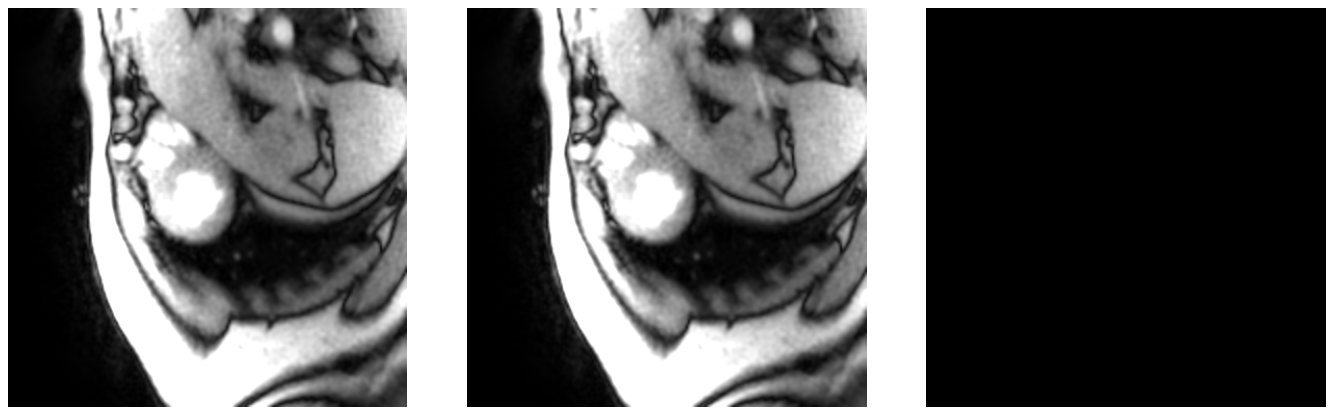

(a) frame $268(11.37 \mathrm{~s})$
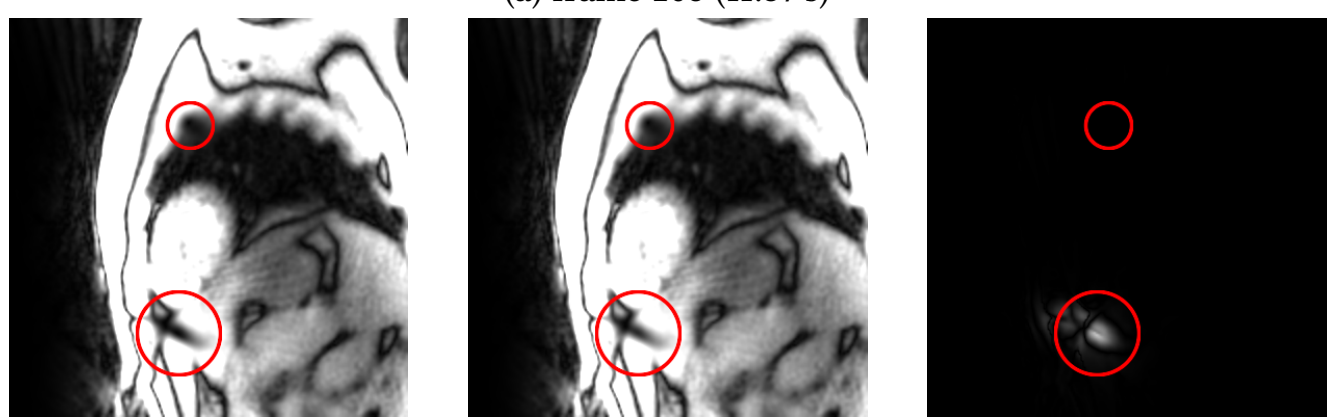

(b) frame $284(12.05 \mathrm{~s})$
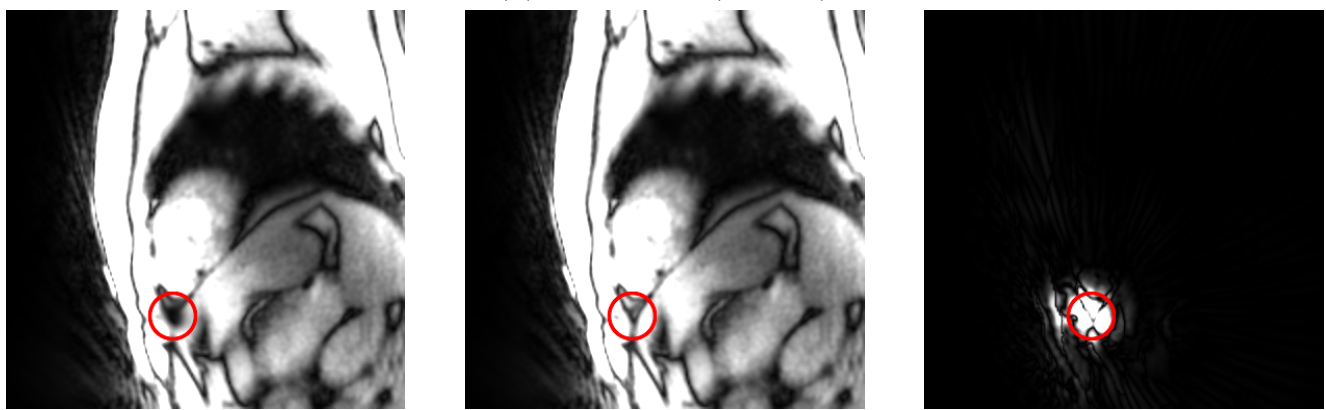

(c) frame $294(12.47 \mathrm{~s})$
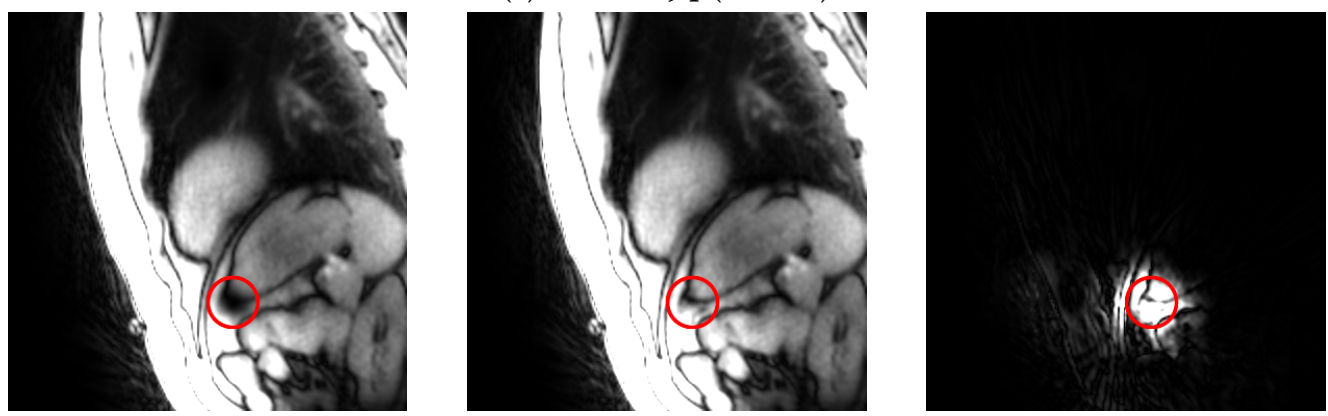

(d) frame $398(16.88 \mathrm{~s})$

Figure 4.14: The same reconstruction as Figure 4.13 windowed to highlight regions of low intensity. Left: real-time NLINV ENLIVE with 1 map), center: ENLIVE with 2 maps, right: their difference scaled up by $\times 5$. This allows easier identification of the signal voids indicated by the red circles. 



\section{Nonlinear Reconstruction with Additional Spatial and Temporal Dimensions}

Since MRI is an inherently slow technique, motion is a common problem. This can be physical motion such as breathing or cardiac motion, signal response changes due to injection or ingestion of MRI contrast agents, or even movement of the acquired slice in dynamic MRI.

The challenges that motion poses in MRI can be illustrated by considering the example of cardiac exams:

While breathing motion can be suppressed by, e.g. breath-hold exams, cardiac motion cannot be stopped. Furthermore, this cardiac motion is of interest in itself, since it contains information about the function of the heart, and it is often the primary reason for performing cardiac MRI Studying the cardiac motion in real time is especially important in the presence of arrhythmia.

In general, avoiding breath-holds is desirable because it eliminates a source of error, since children and otherwise non-compliant patients often fail to hold their breath. This is particularly true for patients with heart problems, because these patients are often unable to hold their breath.

Therefore, approaches are needed which ideally resolve both breathing and cardiac motion. Using a highly undersampled and thereby very fast acquisition can provide data with a sufficient time-resolution to resolve heart dynamics as well as breathing motion 8 30].

As for reconstructing these data, there are multiple possible approaches, each making different assumptions: There are approaches close to classical CINE MRI based on synchronization with an electrocardiogram (ECG) or on self-gating, i.e. extracting cardiac and respiratory phase from the MRI signal, sorting the data according to these states, and reconstructing the resulting multidimensional dataset. This approach assumes periodicity in the cardiac and respiratory states in different cycles. Two examples of such approaches 
are Feng et al.'s [84] XD-GRASP (extra-dimensional GRASP)] and the method published by Cheng et al. [85]. An advanced method for self-gating for use with such methods is Rosenzweig et al.'s 76 SSA-FARY (singular spectrum analysis for advanced reduction of dimensionalty), which will be used later.

Without this assumption about states, the data can be reconstructed as a time series, for example by binning a few excitations into time frames and reconstructing these. Here, the assumption is that adjacent frames contain redundant information and that can be used to overcome undersampling. Both of these approaches can only be used after acquisition has finished, and are therefore no suitable for real-time reconstruction.

Such data can also be reconstructed using a causal reconstruction, that means with a method which only takes into account already acquired data. This still comes with the assumption about redundancy in adjacent time frames, but it enables reconstruction concurrent with the acquisition, which both of the aforementioned methods exclude. Such methods are therefore the only ones which can enable real-time (i.e. concurrent with the acquisition) reconstruction (e.g. using NLINV] see [8, 30]). Furthermore, as only past data, and often only the previous frame, is used for reconstruction, these methods only slightly increase the problem size.

For non-periodic motion, such as movement of the slice position or orientation during a scan, or the perfusion of a contrast agent through the body, only reconstruction as a time series is feasible.

Additionally, since modern MRI generally involves multiple coils, coil sensitivity profiles accurate over the entire dataset are necessary for all three approaches. If they can be assumed to be constant over the dataset, it is simple to pre-calculate sensitivities. However, in the case of varying coil profiles, pre-calculation becomes difficult or infeasible. In such cases, methods which simultaneously estimate image content and coil sensitivity profiles are preferable, such as NLINV

In the following chapter, I introduce a technique which can be used for reconstruction of both self-gated data and of time series. This method fully exploits and embraces the multi-dimensional nature of such datasets, combining the accurate calculation of multidimensional coil profiles and images through non-linear inversion with the quality improvements of compressed sensing.

This technique, termed XD-NLINV is applied to a variety of MRI techniques, starting with dynamic contrast-enhanced data first and then applied to different fast acquisitions of the head and the heart, along with self-gated cardiac data.

${ }^{1}$ a multidimensional extension of golden-angle radial sparse parallel MRI (GRASP) 


\subsection{Theory and Implementation}

Here, we assume data from $N_{C}$ coils, each consisting of measurements at multiple timepoints $t$, are stacked into a vector $Y_{t}$. Then the time-series of images $m_{t}$ and of the spatial coil sensitivities $C_{t}$ can be recovered by solving

$$
\underset{m_{t}, C_{t}}{\arg \min }\left\|Y_{t}-\mathcal{P}_{t} \mathcal{F} C_{t} m_{t}\right\|_{2}^{2}
$$

Here, $\mathcal{F}$ is the $(2 \mathrm{D}$ or $3 \mathrm{D})$ Fourier transform at each time point and $\mathcal{P}_{t}$ the projection onto the acquired pattern/trajectory in k-space.

Since this problem is highly ill-posed, and to exploit compressed sensing regularization is added. As with regular NLINV this will include a term penalizing high spatial frequencies in the coil profiles. In principle, this problem can also be solved using the IRGNM However, I chose an alternating minimization scheme instead. Alternating minimization has been used before in MRI 29], and one of its qualities is the ease with which different regularization can be added to the subproblems, since they are linear inverse problems. This is especially appealing in MRI since the prior knowledge about images and coil profiles is different, as coil profiles are, for example, known to be smooth in space while the images, in general, are not. Therefore, the regularization applied to either will, in general, also be different. While this could be added to an implementation based on the IRGNM as well ${ }^{2}$, it is simpler in an alternating minimization formulation, which is why it is used here.

In order to apply an alternating minimization scheme, we observe that the problem Equation (5.1) is bilinear: with fixed $m_{t}$ it is linear in $C_{t}$ and vice versa. This allows a natural decomposition as two linear inverse problems that have to be solved in tandem:

$$
\begin{array}{crc}
\underset{C_{t}}{\arg \min }\left\|Y_{t}-A_{C t} C_{t}\right\|_{2}^{2}+\alpha R_{1}\left(C_{t}\right) & \underset{m_{t}}{\arg \min }\left\|Y_{t}-A_{m t} m_{t}\right\|_{2}^{2}+\beta R_{2}\left(m_{t}\right) \\
& \text { with: } A_{C t}=\mathcal{P}_{t} \mathcal{F} m_{t} & \text { with: } A_{m t}=\mathcal{P}_{t} \mathcal{F} C_{t}
\end{array}
$$

In case of simple Tikhonov-type $\ell_{2}$-regularization, both subproblems can, for example, be solved with the conjugate gradient method (CG) [86]. In case of a compressed sensing reconstruction with, e.g. $\ell_{1}$-wavelets or total variation (TV) regularization, the problem becomes non-smooth. Therefore, different reconstruction algorithms are needed which can properly include such terms, such as FISTA (fast iterative shrinkage/thresholding algorithm) 32] or ADMM (alternating direction methods of multipliers) [33 34]. For this thesis, all XD-NLINV reconstructions were performed with ADMM.

As a post-processing step, image and coil sensitivity profiles are multiplied to generate

${ }^{2}$ And this is indeed planned for the future. 
coil images for all time points, which are then combined by calculating their root-sum-ofsquares, analogous to Equation 4.7) on page 24.

Instead of a single time dimension as shown in Equations 5.1 and (5.2), this can be extended into an arbitrary number of other dimensions, such as additional spatial dimension or cardiac and/or respiratory state.

\subsection{Experiments}

To evaluate this method, I applied it to several datasets spanning dynamic contrast enhanced (DCE) [MRI, "virtual moving table", real-time imaging of the human heart, and self-gated dataset with both cardiac and respiratory motion dimensions.

A DCE dataset of a human liver 3 was acquired at $1.5 \mathrm{~T}$ with: TR/TE: $4.27 \mathrm{~ms} / 1.55 \mathrm{~ms}$, flip angle: $12^{\circ}$, base resolution: 256 , FOV $385 \times 385 \mathrm{~mm}^{2}$, slice thickness: $5 \mathrm{~mm}$. The total dataset comprises 63120 TRs in 30 slices, from which I extracted a single slice which I then binned into frames of 21 TRs each. This dataset was then reconstructed using realtime NLINV and with XD-NLINV The XD-NLINV reconstruction included a temporal total-variation penalty.

As an example of an acquisition with naturally changing coil profiles, I turned to "virtual moving table" MRI Here, the acquisition plane is moved in each frame in a direction orthogonal to the imaged slice, mimicking a movement of the scanner table, albeit with more degrees of freedom. Such acquisitions can be used for fast localization of structures of interest. The coil profiles, of course, change from frame to frame, so constant profiles cannot accurately describe the data. However, XD-NLINV can be used to reconstruct these data. The selected dataset is a $2 \mathrm{D}$ FLASH acquisition of a human volunteer with no known illnesses, starting from the top of the skull in transversal direction and covering the entire head down to the lower jaw. Acquisition parameters are: TR/TE: $3 \mathrm{~ms} / 1.93 \mathrm{~ms}$, flip angle: $15^{\circ}$, FOV $192 \times 192 \mathrm{~mm}^{2}$, resolution: $1 \times 1 \times 5 \mathrm{~mm}^{3}$, slice shift per frame: $1 \mathrm{~mm}$, bandwidth per pixel: $840 \mathrm{~Hz}$ with random[rf] spoiling [24]. 43 uniformly distributed radial spokes per frame were acquired, where 5 successive frames each contain complementary spokes. This leads to a measurement time of $129 \mathrm{~ms}$ per frame and a total measurement time of $24.75 \mathrm{~s}$ for 192 slices (frames). This dataset was then reconstructed using real-time NLINV and using XD-NLINV with a total-variation penalty in the slice (frame) direction and with a spatial wavelet penalty.

Another area where XD-NLINV can potentially improve reconstruction quality is real-

${ }^{3}$ Courtesy of Tobias Block 
time imaging of the human heart. To evaluate XD-NLINV on real-time data, I selected 60 frames from a bSSFP acquisition of the human heart of a volunteer with no known illnesses. Acquisition parameters are TE/TR: $3.32 / 1.66 \mathrm{~ms}$, flip angle: $53^{\circ}$, matrix size: $160 \times 160$, FOV $256 \times 256 \mathrm{~mm}^{2}$, slice thickness: $6 \mathrm{~mm}$. These data were reconstructed using XDNLINV and using ESPIRiT followed by a PICS with a linear model. Both reconstructions included a temporal total-variation constraint.

An example where pre-calculated coil profiles are infeasible is an interactive real-time acquisition: Here, the slice position and rotation is changed during the scan, so precalculated coil profiles cannot be valid for the entire reconstruction. Therefore, a portion of the interactive real-time dataset described in Section 4.3 was extracted and reconstructed using ESPIRiT followed by a PICS with a linear model, using real-time NLINV and using XD-NLINV. Both ESPIRiT+PICS and XD-NLINVreconstructions included a temporal totalvariation penalty, with an additional spatial wavelet regularization for the XD-NLINV reconstruction.

Additionally, an inversion recovery radial FLASH dataset, originally acquired for fast $T_{1}$ mapping 4 was selected to asses XD-NLINV] s performance. This dataset is a short-axis view of a human volunteer with no known illnesses, acquired at $3 \mathrm{~T}$. After an initial adiabatic inversion pulse, 9o frames covering $4.05 \mathrm{~s}$ were acquired. Additional sequence parameters were: TR/TE: $2.67 \mathrm{~s} / 1.67 \mathrm{~s}$, FOV $256 \times 256 \mathrm{~mm}^{2}$, matrix size: $256 \times 256$, slice thickness: $8 \mathrm{~mm}$, flip angle: $6^{\circ}$, bandwidth: $850 \mathrm{~Hz}$ per pixel. These data were then reconstructed using XD-NLINV with a spatial and a temporal total-variation penalty.

Finally, a dataset was binned by SSA-FARY into respiratory and motion states 5 . This is a short-axis view of the human heart, acquired using 26 channels of a thorax coil and a golden-angle radial FLASH trajectory at $3 \mathrm{~T}$. Further acquisition parameters were: TR/TE: $2.60 \mathrm{~ms} / 1.63 \mathrm{~ms}$, flip angle: $12^{\circ}$, matrix size: $192 \times 192$, FOV $256 \times 256 \mathrm{~mm}^{2}$, slice thickness: $7 \mathrm{~mm}$, acquisition time: $30 \mathrm{~s}$. This dataset, consisting of 11539 individual TRs, was binned into 25 cardiac and 9 respiratory states using SSA-FARY [76]. XD-NLINV reconstruction included a total variation penalty in the cardiac and respiratory dimensions together with spatial wavelets. As a comparison, Sebastian Rosenzweig's [76] reconstruction of this dataset was used. There, coil profiles for each breathing state were pre-calculated by ENLIVE followed by a PICS reconstruction with a linear model including a total variation penalty in the cardiac and respiratory dimensions and a spatial wavelet penalty.

Regularization parameters of the ESPIRiT+PICS and XD-NLINV collected in Table A.1 in Appendix A

${ }^{4}$ Courtesy of Xiaoqing Wang

${ }^{5}$ Courtesy of Sebastian Rosenzweig 


\subsection{Results}

Selected frames of the DCE dataset corresponding to pre-contrast, the venous phase, arterial phase and a late frame of the resulting reconstructions can be seen in Figure 5.1 Comparing the two reconstructions, we can see that, while the real-time reconstruction exhibits slight blurring, the XD-NLINV is free of blurring. This can be quantified by analyzing the signal enhancement in time: Figure 5.2 shows that signal enhancement for both reconstruction methods. Here, both XD-NLINV and real-time NLINV follow a similar time course. The time curves for different strengths of the total-variation penalty in XD-NLINV are shown in Figure 5.4. There we can see that increasing the total-variation penalty also increases smoothness of the signal curve in XD-NLINV

The coil profiles calculated by XDNNINV corresponding to the frames in Figure 5.1 are shown in Figure 5.6. For easier visualization, the 6 profile are combined as the root-sumof-squares of the individual coil profiles. These coil profiles exhibit some of the effect of the contrast-agent induced intensity increase, showing that the XDNLINV model does not perfectly separate image and coil sensitivities.

Selected slices of the "virtual moving table" reconstruction are shown in Figure 5.7 Here, while XD-NLINV reconstructs this dataset, no major improvement in quality is visible. On the contrary, the real-time NLINV reconstruction seems to show superior in-plane (transversal) image quality. For the sagittal and coronal slices, XD-NLINV shows less blurring. Close to the spine, this dataset shows a periodic artifact with a period of about $750 \mathrm{~ms}$. This is likely due to blood flow, as $750 \mathrm{~ms}$ would correspond to a heart rate of 80 beats per minute, which is in the expected range for an adult at rest.

Selected frames of the XD-NLINV reconstruction of the inversion recovery FLASH dataset are shown in Figure 5.8, with the time curves of three different tissue types shown in Figure 5.9. XD-NLINV faithfully reconstructs these data, showing the contrast inversion between blood and myocard in Figure 5.8(d) compared to Figure 5.8(b) and Figure 5.8(f) as well as the lack of contrast visible in Figure 5.8(e)

In the real-time cardiac bSSFP reconstruction shown in Figure 5.10, straight nuFFT leads to unusable image quality, with strong streak artifacts overlaying the entire image. However, both XD-NLINV] and ESPIRiT+PICS reconstruction show high quality. Here, $\mathrm{XD}$-NLINV resolved more of the blood vessel in the lung, showing better resolution of small details.

Figure 5.11 shows a comparison of ESPIRiT+PICS real-time NLINV, and XD-NLINV on part of an interactive real-time dataset. The flipped orientation of the first images is due to the slice movement and rotation in interactive real-time. While the image quality for 
the first frame (Figure 5.11(b)) is similar for all three methods, with only real-time NLINV showing slight blurring, the ESPIRiT+PICS reconstruction shows string streak artifacts and signal loss in the second frame (Figure 5.11(b) . Additionally, real-time NLINV exhibits a phase singularity leading to a signal void below the right ventricle (see Section 4.3 for more details on this phenomenon).

Finally, Figure 5.12 shows the results of the cardiac dataset gated with SSA-FARY for four different cardiac and respiratory states. As can be seen, both the PICS reconstruction with a linear model and XD-NLINV resolve the papillary muscles inside of the left ventricle as well as the heart wall, indicating excellent quality of the reconstruction. This is also indicated by their small difference shown in the right column of Figure 5.12 

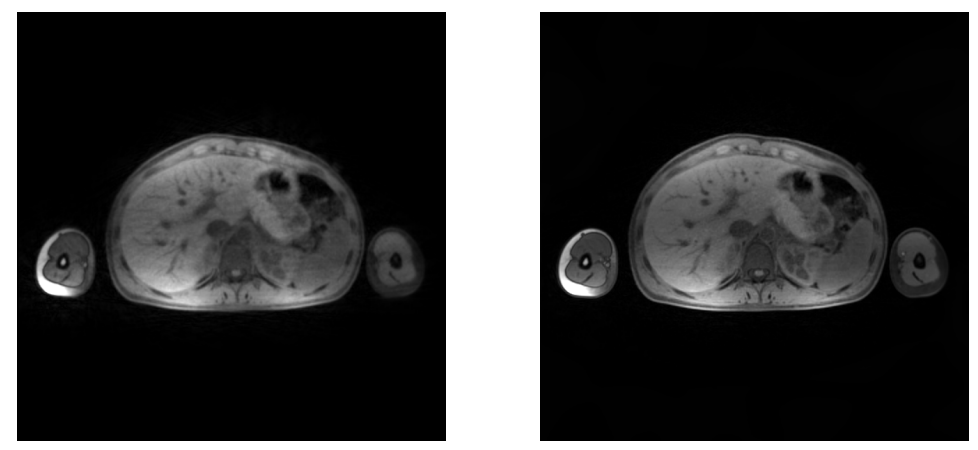

(a) pre-contrast (0s)
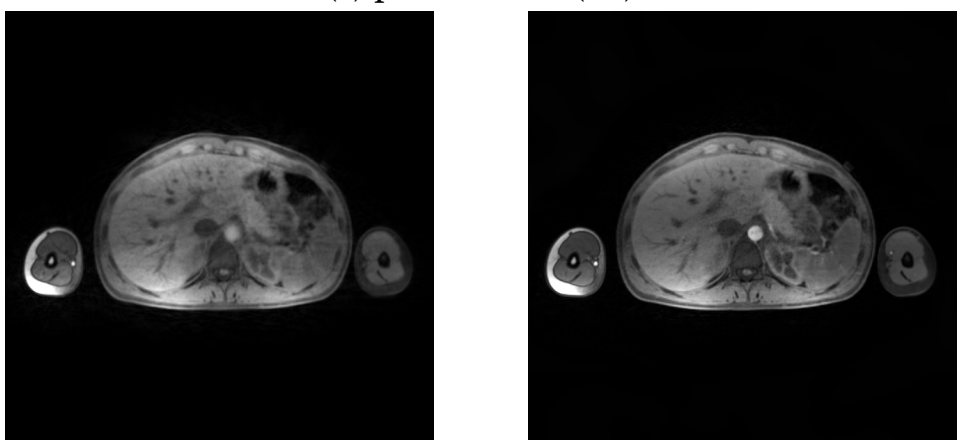

(b) arterial phase (11 s)
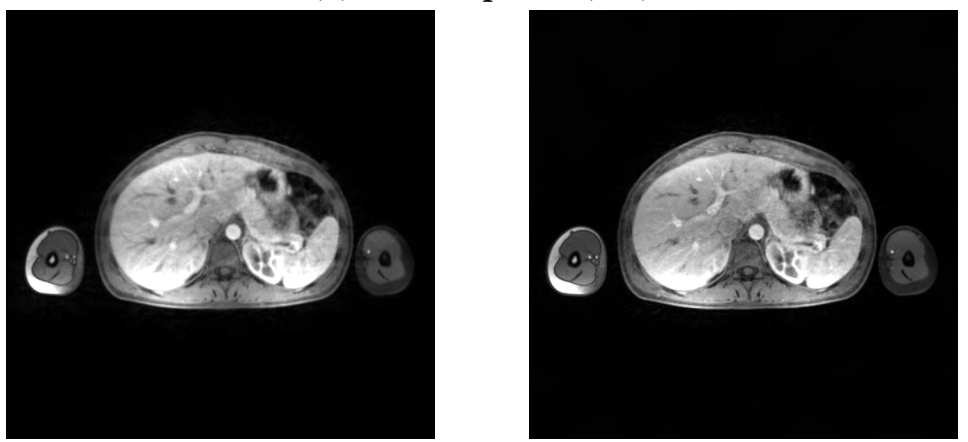

(c) venous phase $(30 \mathrm{~s})$
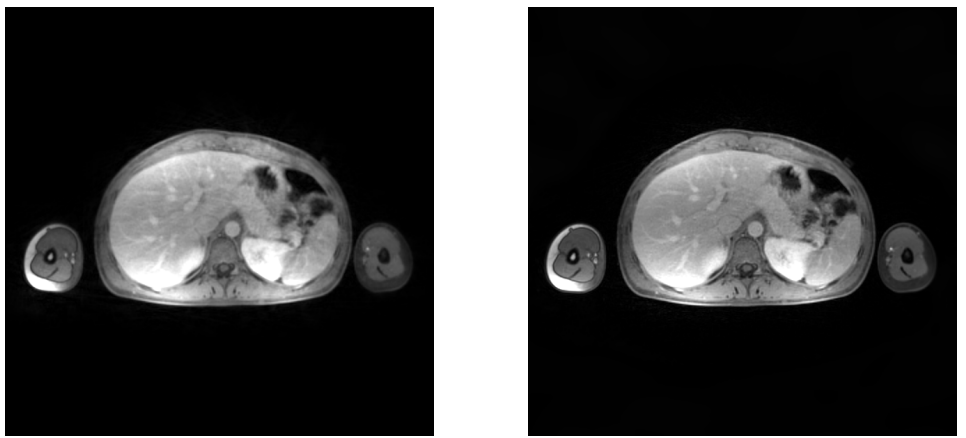

(d) late phase (110 s)

Figure 5.1: Selected frames of a DCE acquisition reconstructed with real-time NLINV (left) and with XD-NLINV including a temporal total-variation constraint (right).(a) pre-contrast,(b) arterial phase,(c) venous phase,(d) late phase. The times are time differences to the pre-contrast image. While the realtime NLINV reconstruction shows blurring, XD-NLINV show excellent image quality. 


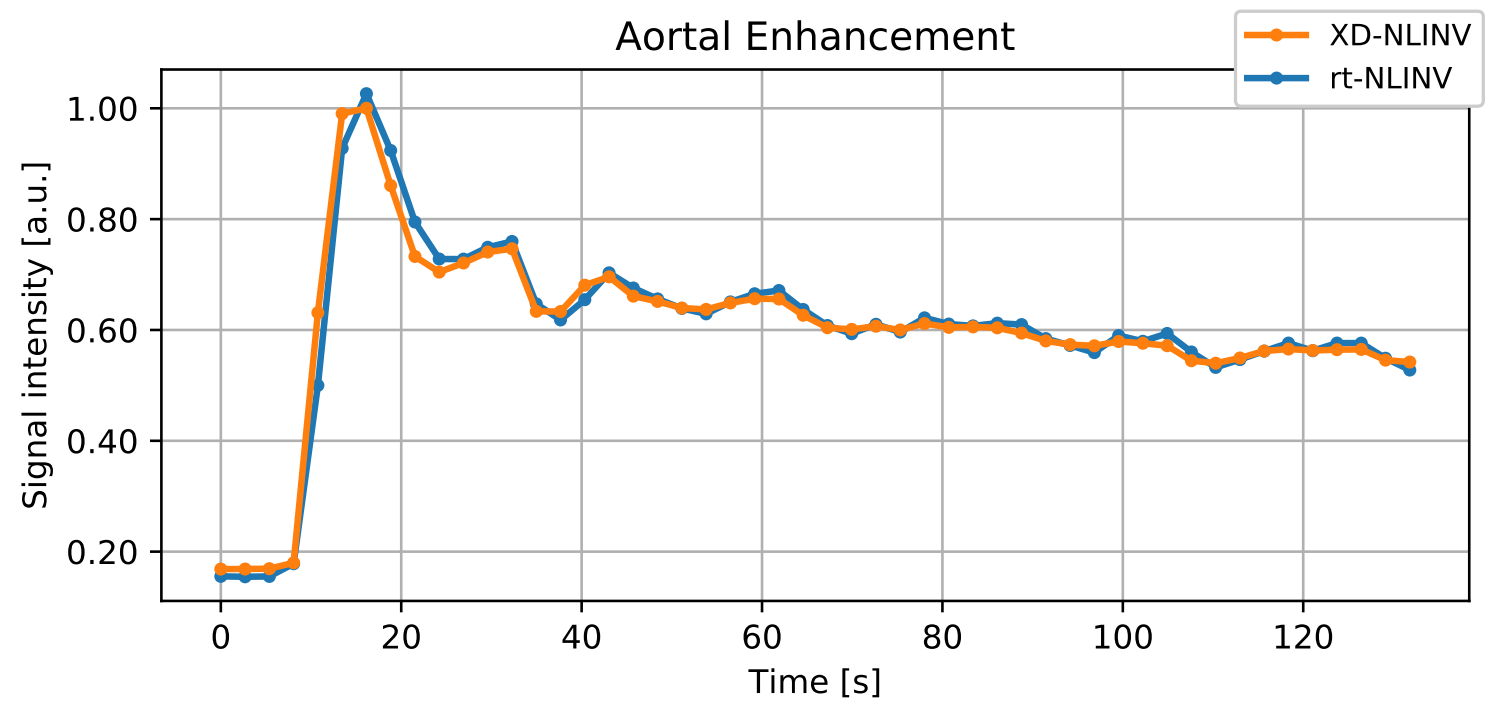

Portal Vein Enhancement

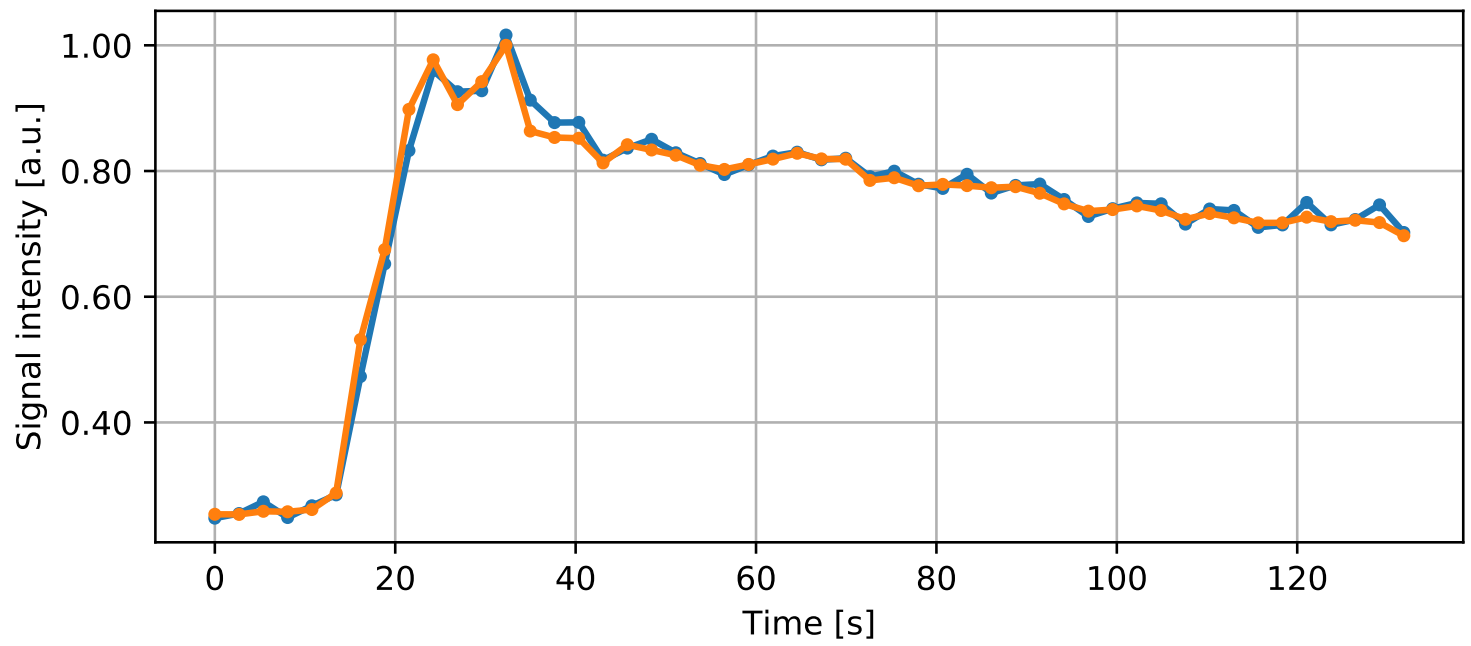

Figure 5.2: Signal enhancement in the aorta (top) and the portal vein (bottom) for real-time NLINV and XD-NLINV Both XD-NLINV and real-time NLINV follow a similar curve. For better visualization of the individual curves, they are shown with offsets in Figure 5.3. 

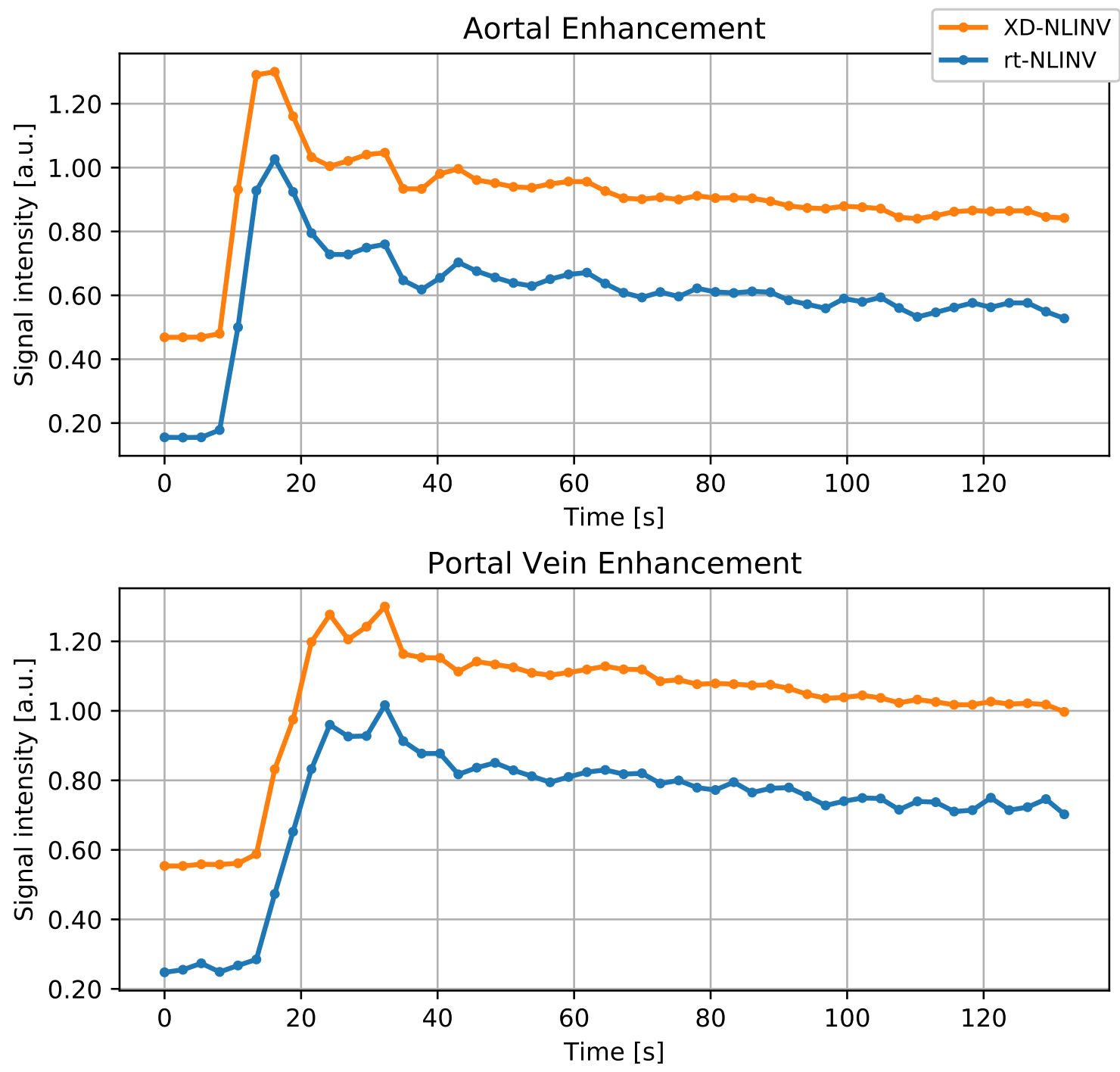

Figure 5.3: The same signal enhancements as in Figure 5.2 shown with a vertical offset. 

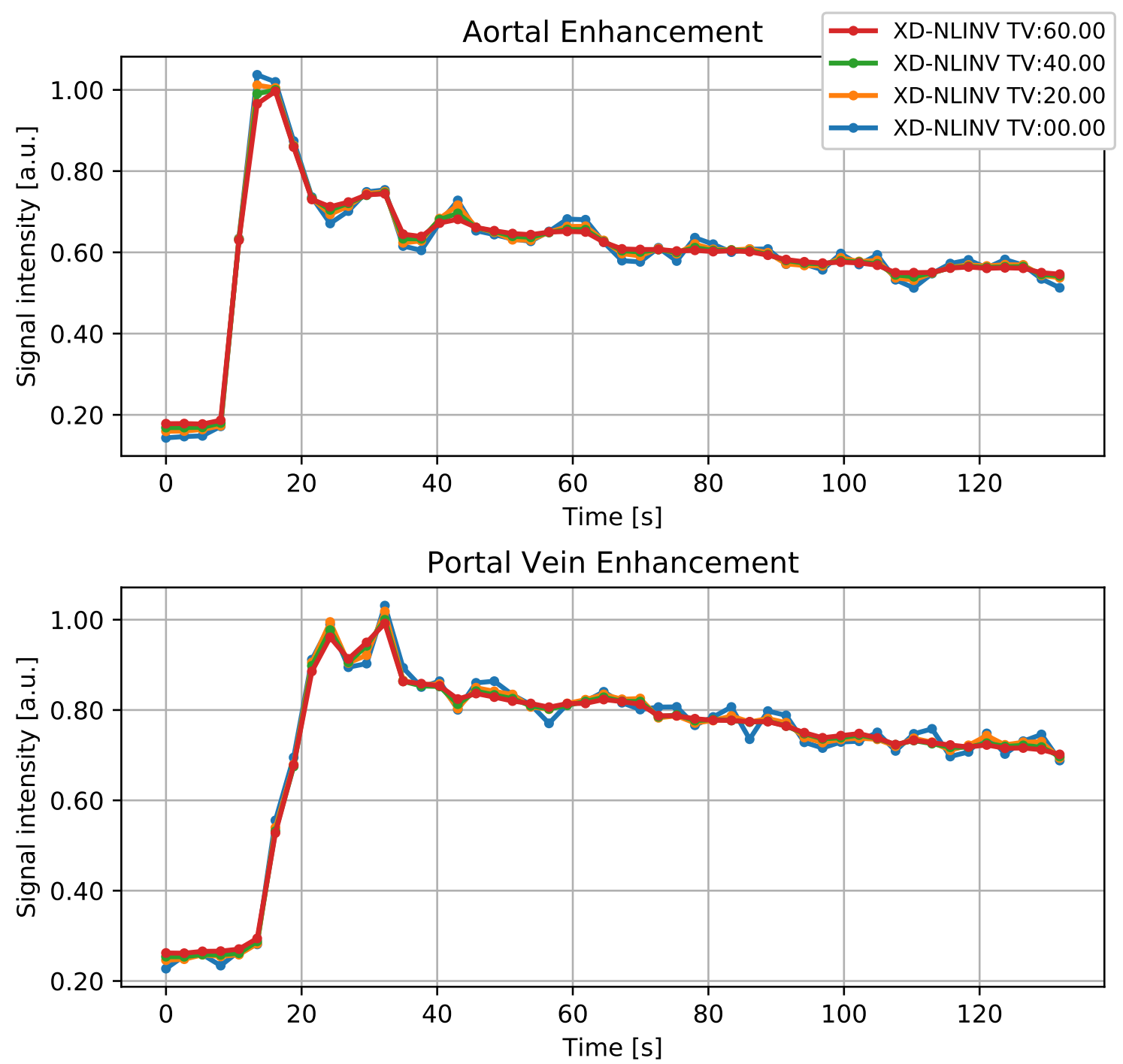

Figure 5.4: Signal enhancement in the aorta (top) and the portal vein (bottom) for XD-NLINV] with different regularization parameters for the temporal totalvariation term. With increasing total-variation penalty, the XD-NLINV shows increasing smoothness as well, smoothing out maxima and minima. The individual curves are shown with a vertical offset in Figure $5 \cdot 3$ 

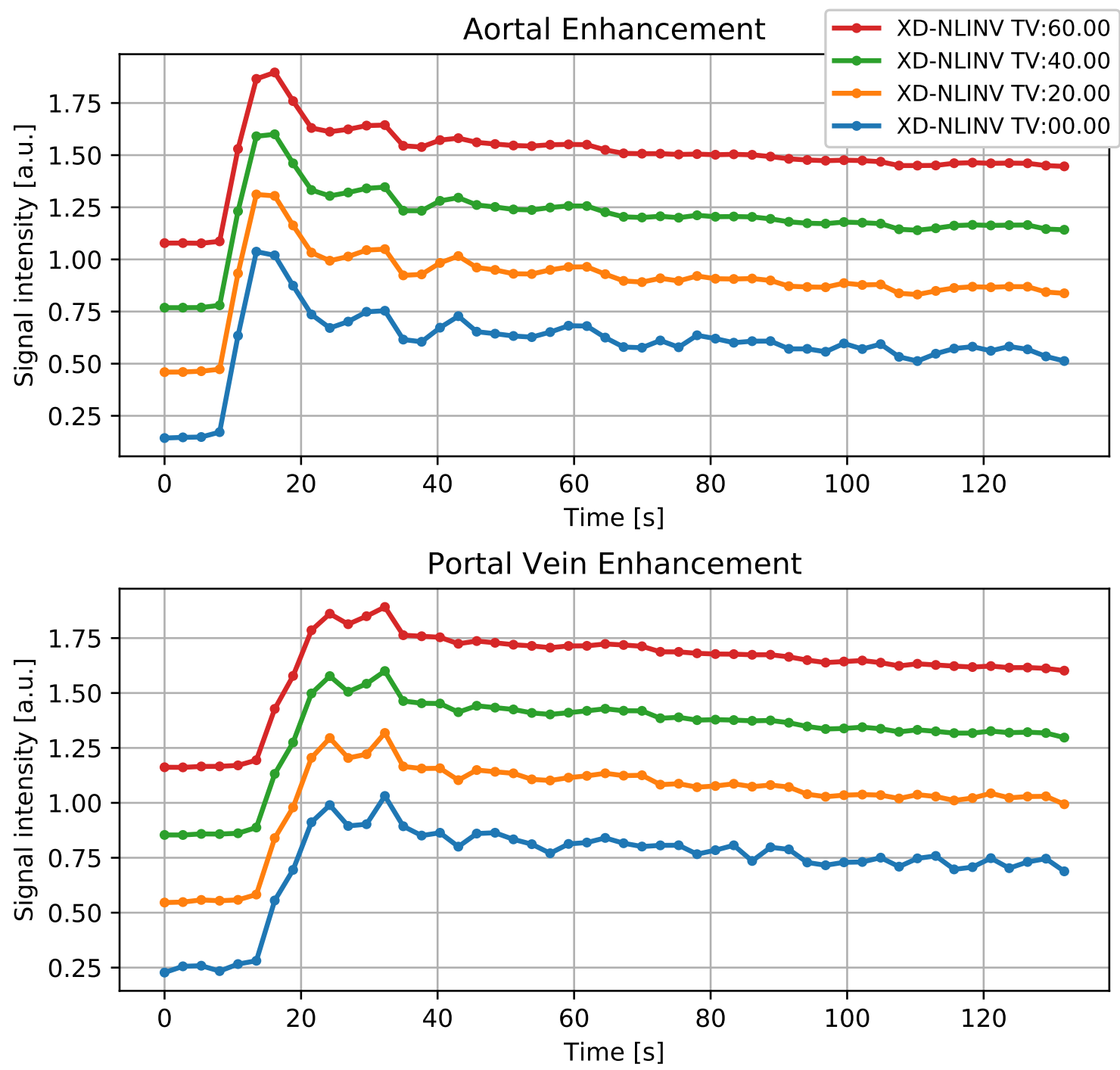

Figure 5.5: The same signal enhancements as in Figure 5.4 shown with a vertical offset. As the total-variation penalty decreases from top to bottom, the curve becomes less smooth. 


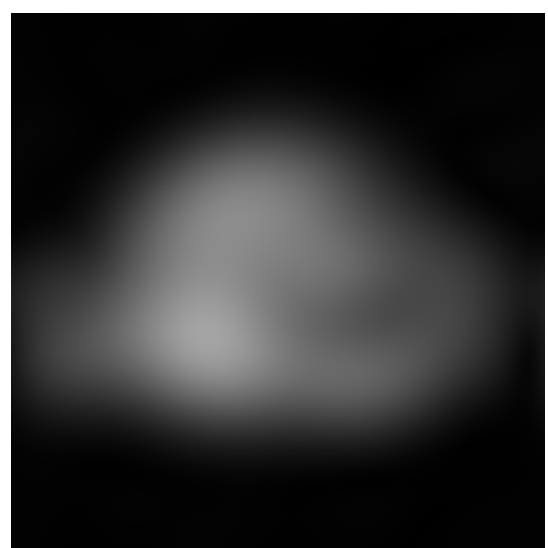

(a) pre-contrast (0 s)

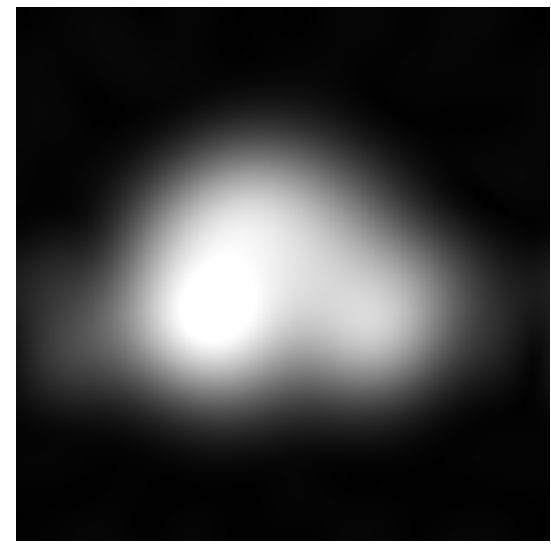

(c) venous phase $(30 \mathrm{~s})$

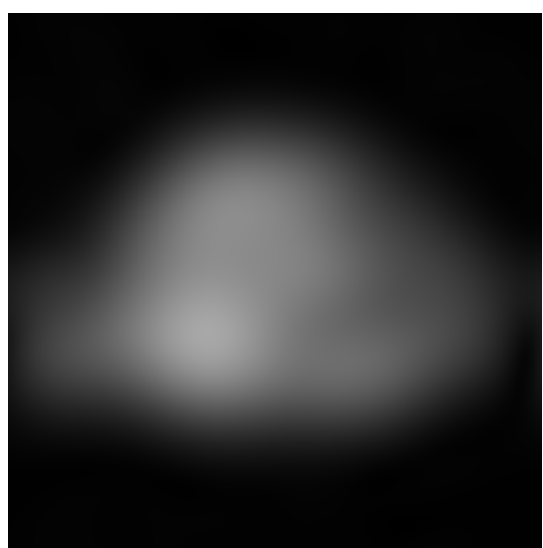

(b) arterial phase (11 s)

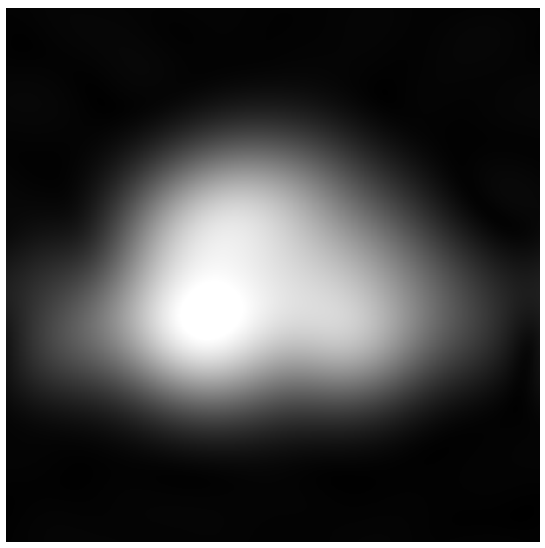

(d) late phase (110 s)

Figure 5.6: Root-sum-squares of the coil sensitivity profiles calculated by XD-NLINV for the DCE dataset shown in Figure 5.1. While overall similar in shape, variations can be seen at the different time points, showing that XDNLINV attributes some intensity variation to the coil sensitivity profiles. All images shown with the same absolute windowing. 

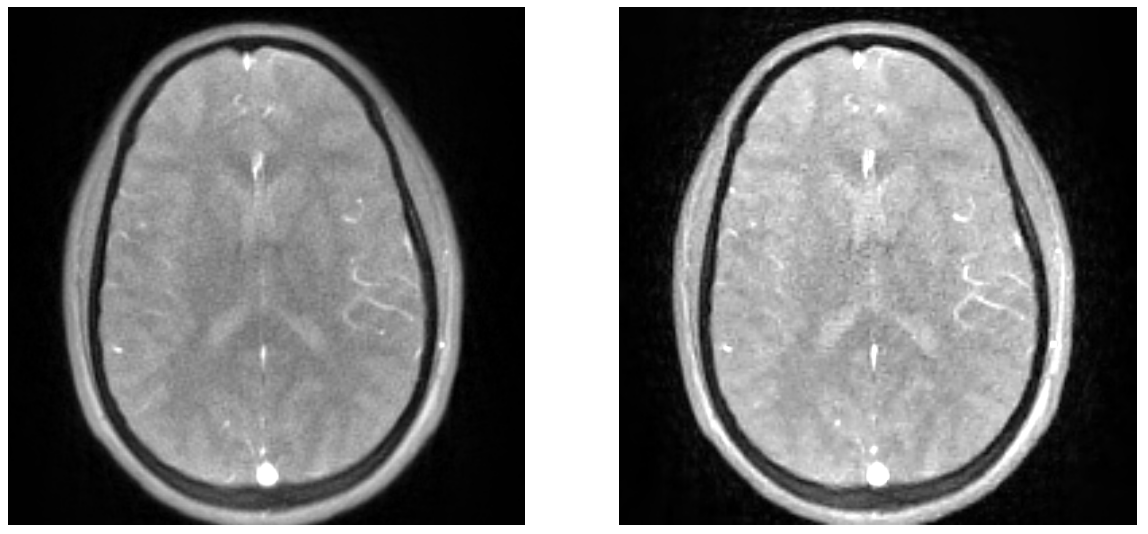

(a) transversal
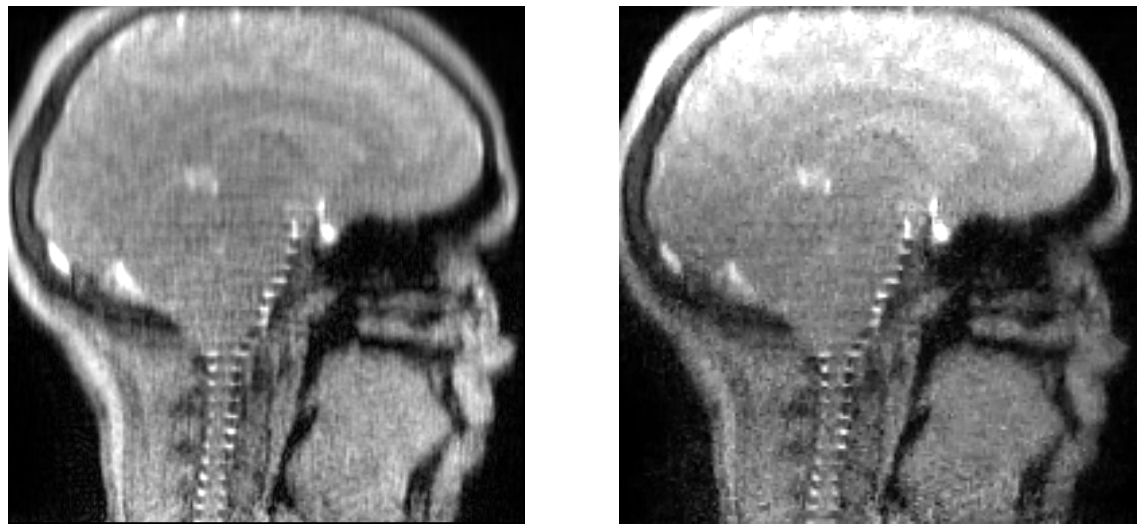

(b) sagittal
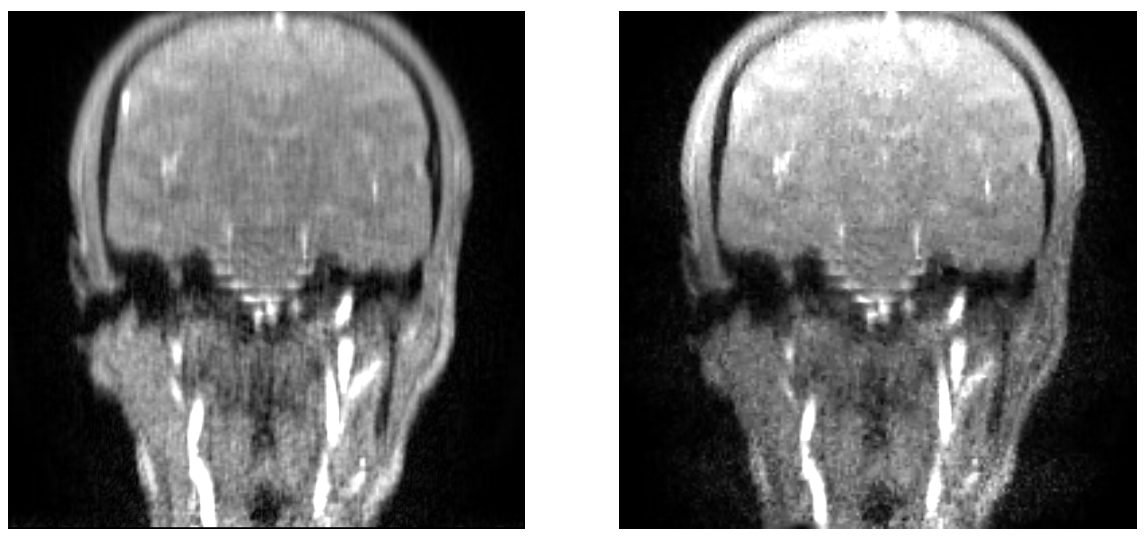

(c) coronal

Figure 5.7: Selected orthogonal slices of the "virtual moving table" dataset reconstructed with real-time NLINV (left) and XD-NLINV (right). The XDNLINV reconstruction included a total-variation penalty in the slice direction (superior-inferior) and a spatial wavelet penalty in the transversal plane. In the transversal slice (a) XD-NLINV does not appear to improve image quality. In the sagittal and coronal slices, XD-NLINV shows less blurring in the superior-inferior direction. Both reconstructions show a periodic artifact close to the spine, with a period of about $750 \mathrm{~ms}$. This is likely due to blood flow, as it would correspond to a heart rate of 80 beats per minute, well within the expected range for an adult at rest. 


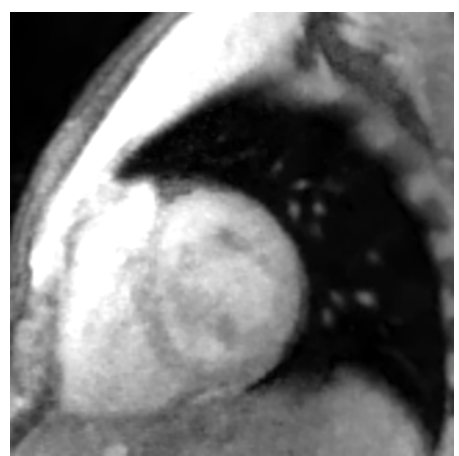

(a) Frame $1(0.06 \mathrm{~s})$

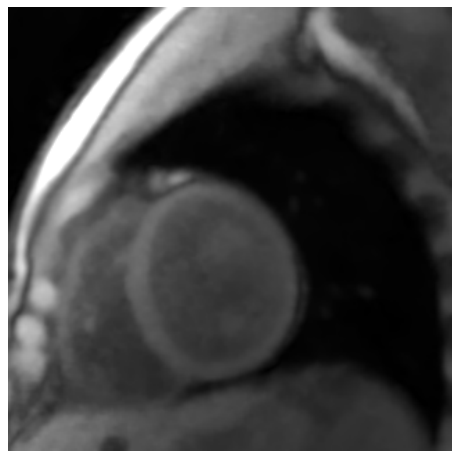

(d) Frame 40 (1.83 s)

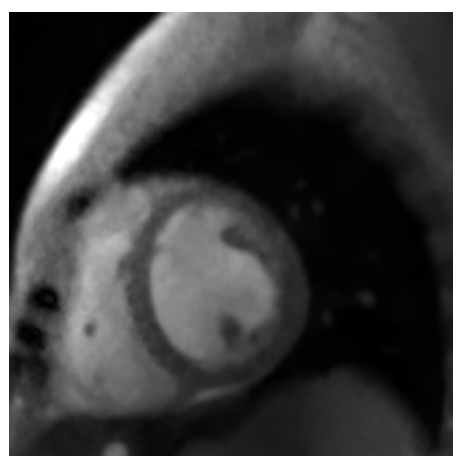

(b) Frame 8 (0.37 s)

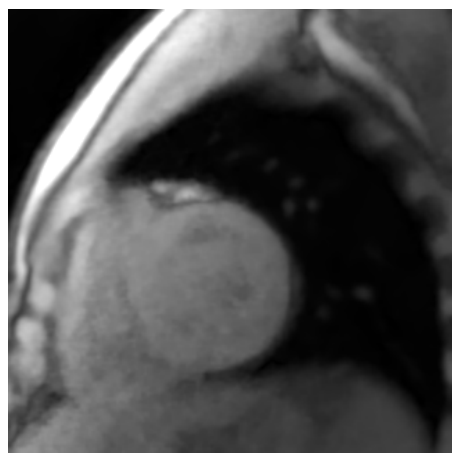

(e) Frame $57(2.60 \mathrm{~s})$

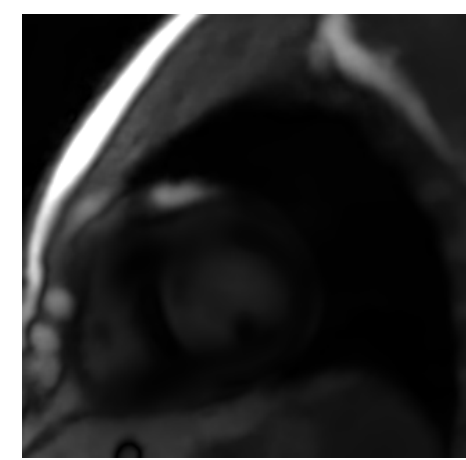

(c) Frame $20(0.92 \mathrm{~s})$

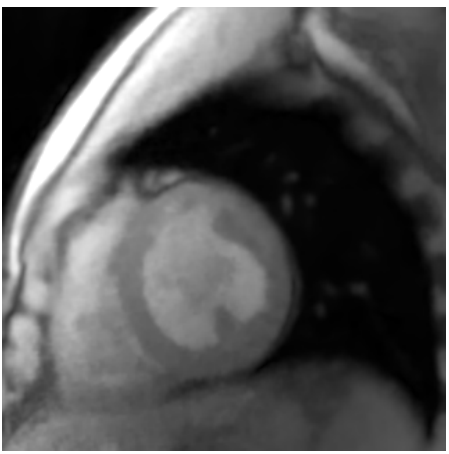

(f) Frame $72(3.28 \mathrm{~s})$

Figure 5.8: Selected frames of the XD-NLINV reconstruction of the inversion recovery radial FLASH dataset. Times after inversion are given in parentheses. This reconstruction includes both a spatial and a temporal total-variation penalty. The differing relaxation times of different tissue types is visible in the distinct contrast changes: For example, there is a contrast reversal between blood and myocard between frames (d) and (f). 


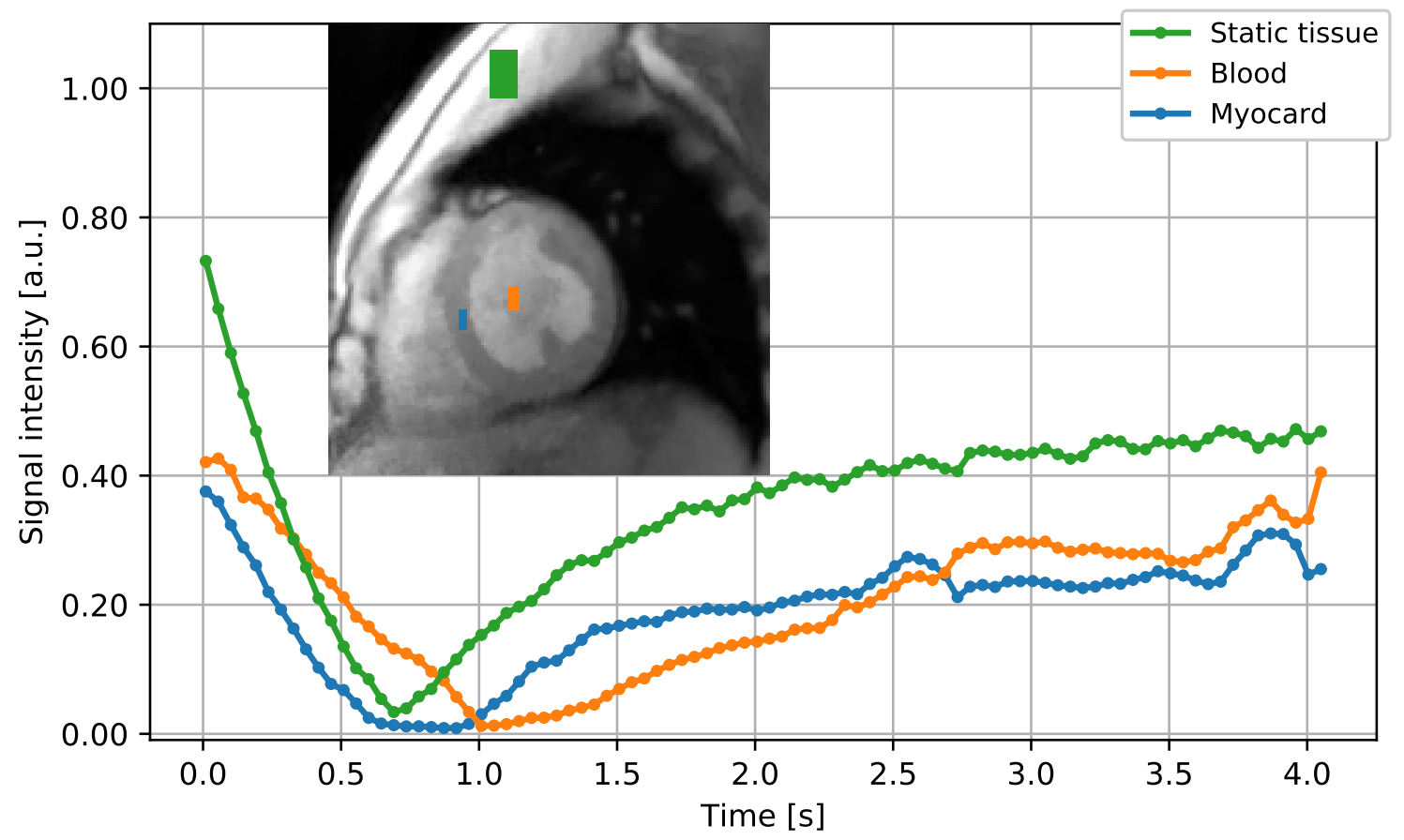

Figure 5.9: Signal development over time in static tissue, in blood inside the left ventricle, and in myocardial tissue. The selected regions of interest are highlighted in the inset. The contrast reversal between blood and myocard at about $2.6 \mathrm{~s}$ after inversion is also visible in Figure $5.8(\mathrm{~d})$ to Figure $5.8(\mathrm{f})$ 

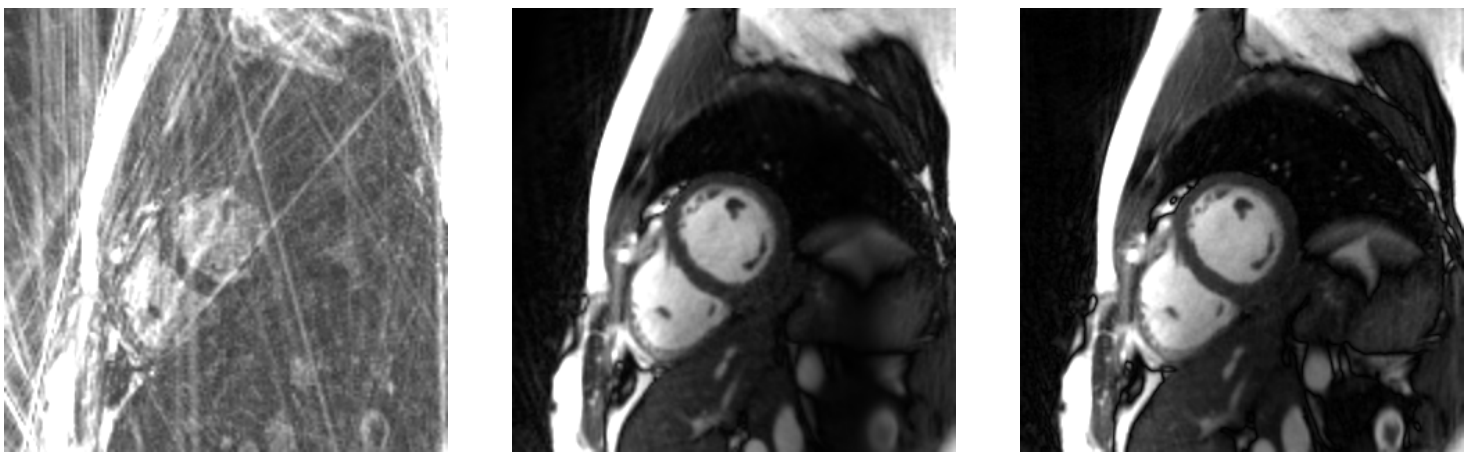

(a) end-diastole
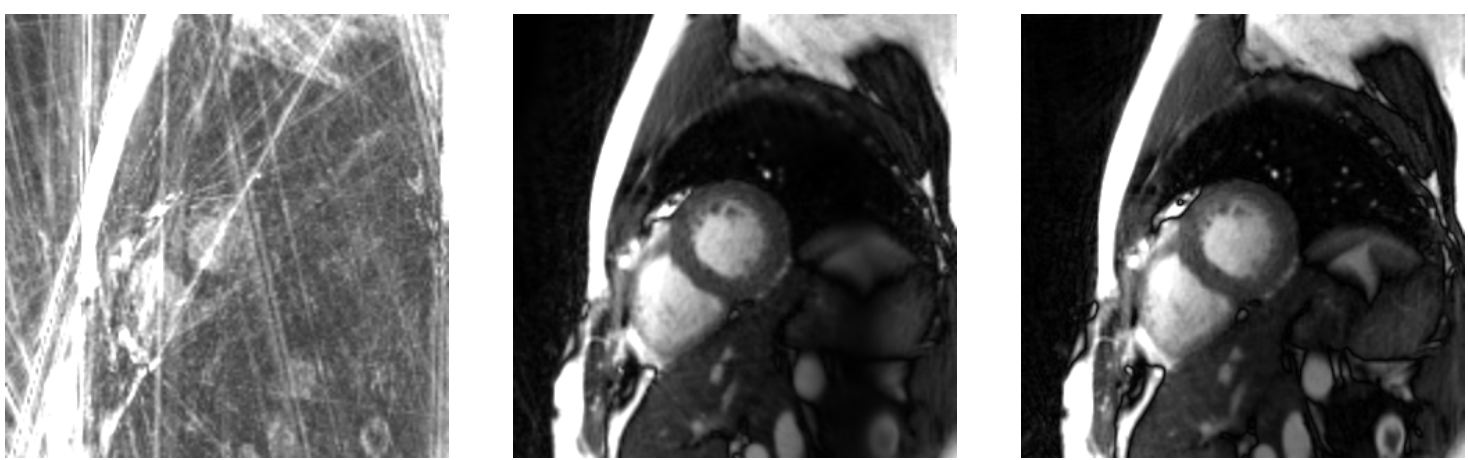

(b) end-systole
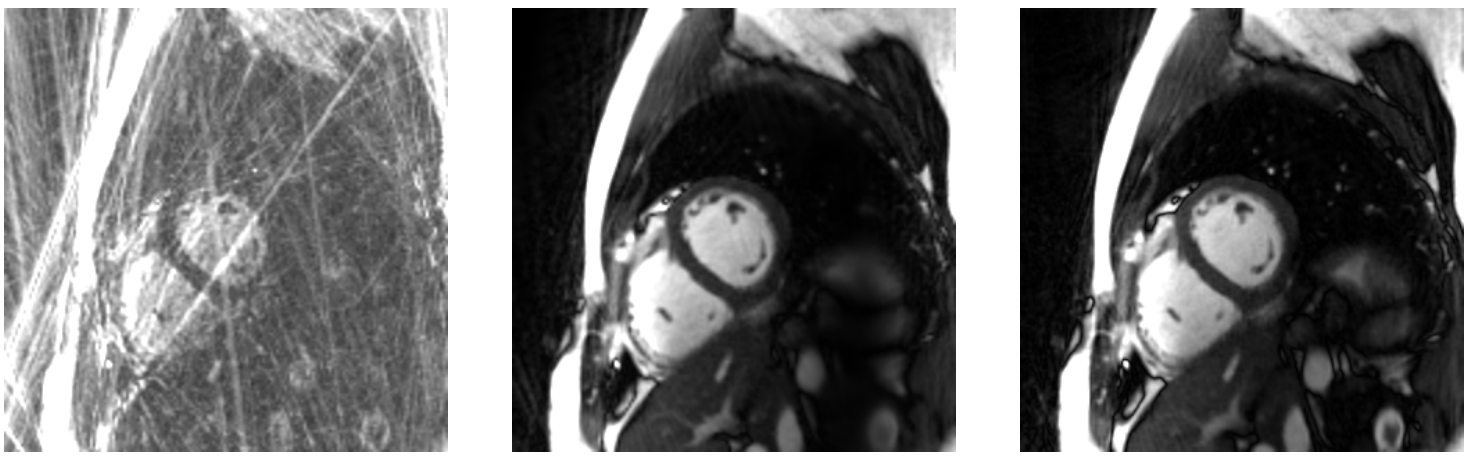

(c) end-diastole (different cardiac cycle)

Figure 5.10: Selected frames of a cardiac real-time bSSFP dataset reconstructed using a ramp-filtered nuFFT (left), ESPIRiT followed by a PICS reconstruction with a linear model (center) and using XD-NLINV (right). Both ESPIRiT+PICS and XD-NLINV reconstructions include a total-variation penalty in time. Direct nuFFT leads to unusable results, but there is little difference in image quality in the heart for ESPIRiT+PICS and XD-NLINV However, the XD-NLINV reconstruction resolves more of the blood vessels in the lung. 

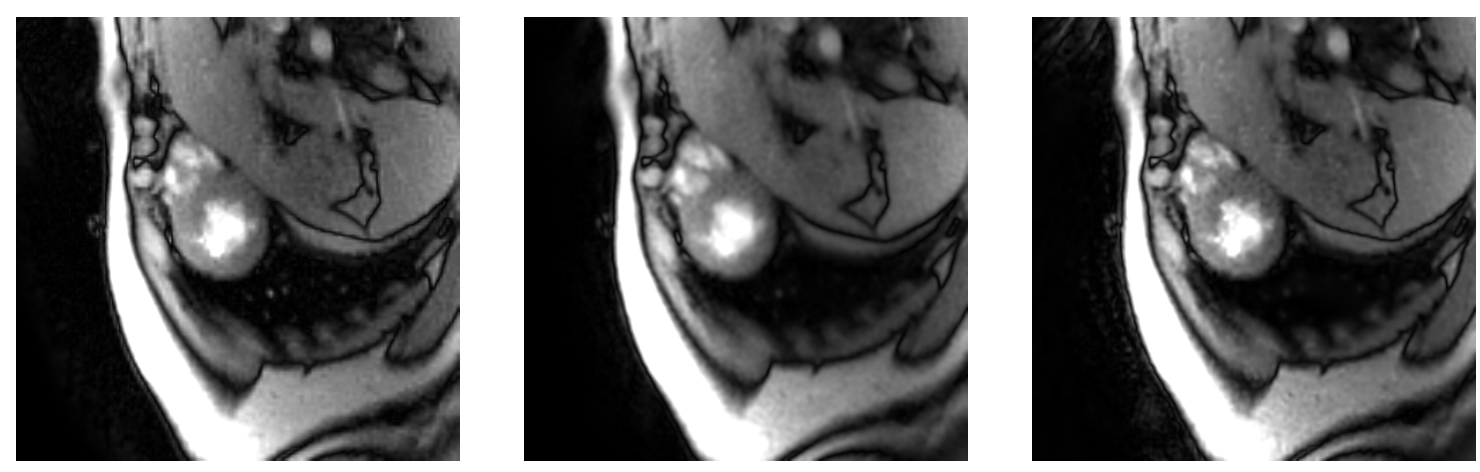

(a) frame $268(11.37 \mathrm{~s})$
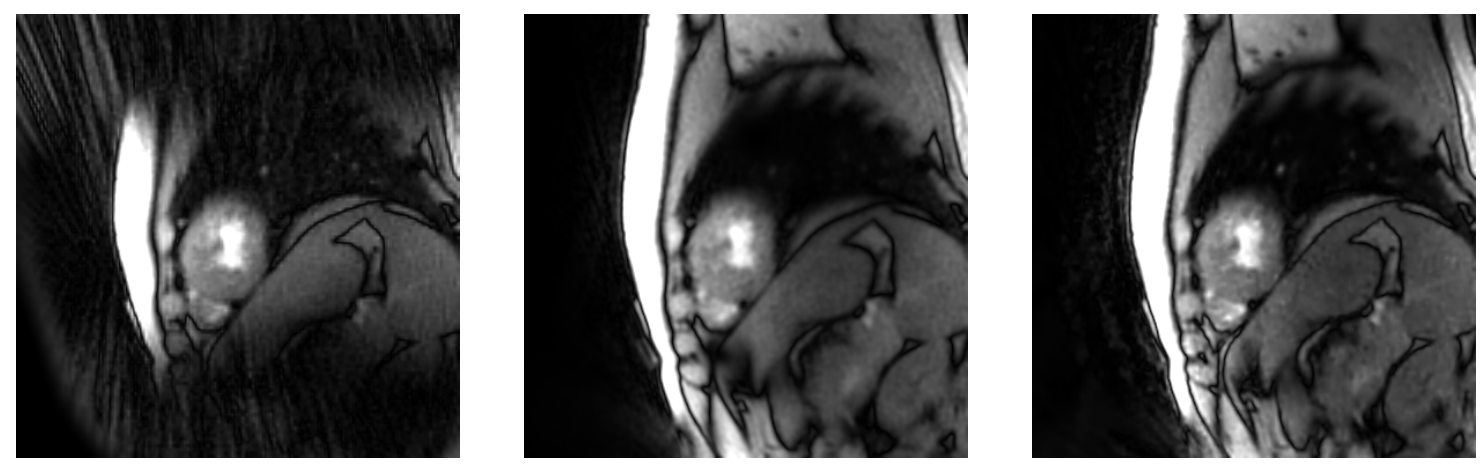

(b) frame $310(13.15 \mathrm{~s})$

Figure 5.11: Selected frames of the reconstruction of an interactive real-time acquisition with ESPIRiT followed by a PICS reconstruction with a linear model (left), real-time NLINV (center), and XD-NLINV (right). The PICS and the XD-NLINV reconstruction included a total variation penalty in time, with an additional a spatial wavelet penalty for the XD-NLINV reconstruction. The real-time NLINV reconstruction shows slight blurring, while XD-NLINV resolves more detail, such as blood vessels in the lung. ESPIRiT+PICS shows good image quality in (a) but fails in (b) This is due to the static coil profiles, which do not describe the data recorded after slice rotation. The unusual rotation of (a) is due to the free movement and rotation of the slice during acquisition. Real-time NLINV additionally shows a signal void in (b) below the apex of the heart, more easily seen in Figure $4.13(\mathrm{c})$. 

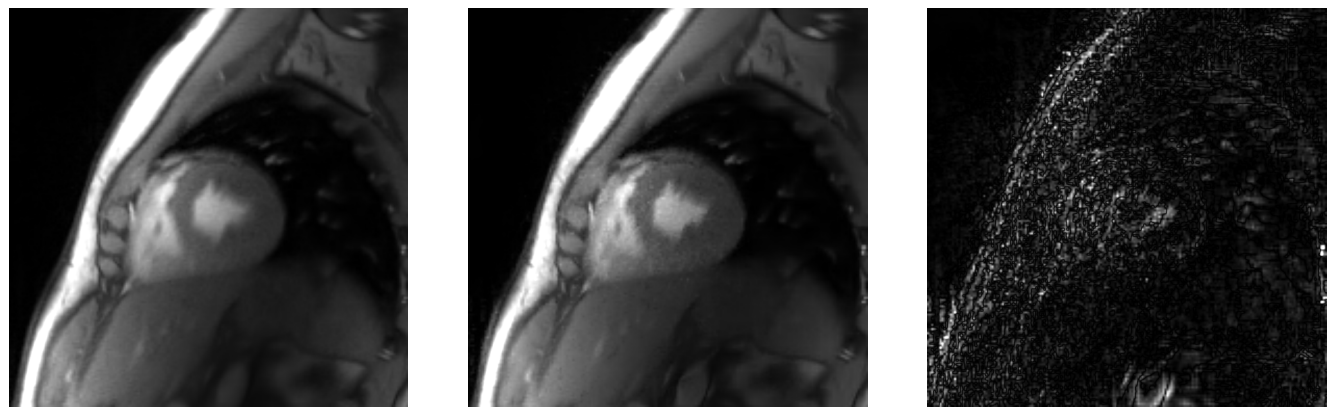

(a) end-systole end-inhalation
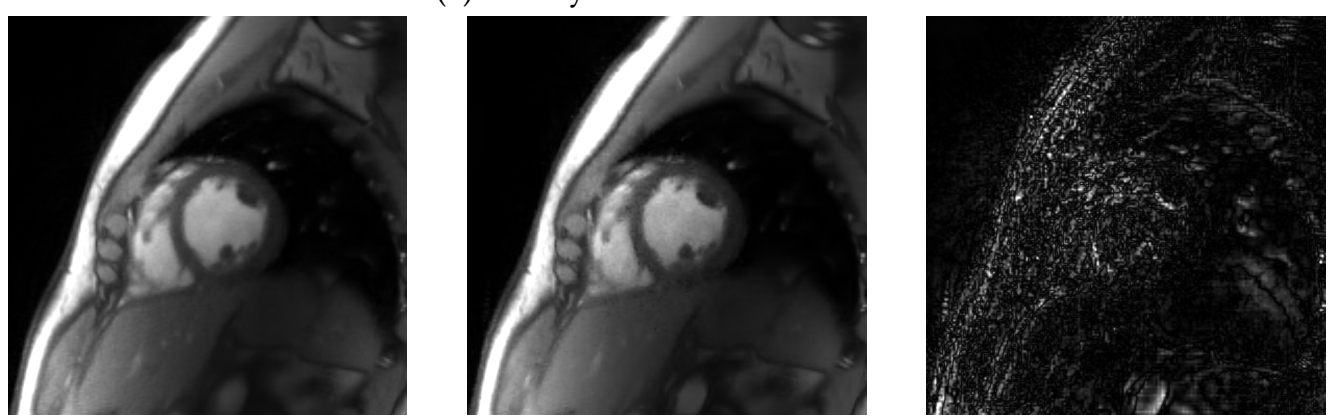

(b) end-diastole end-inhalation
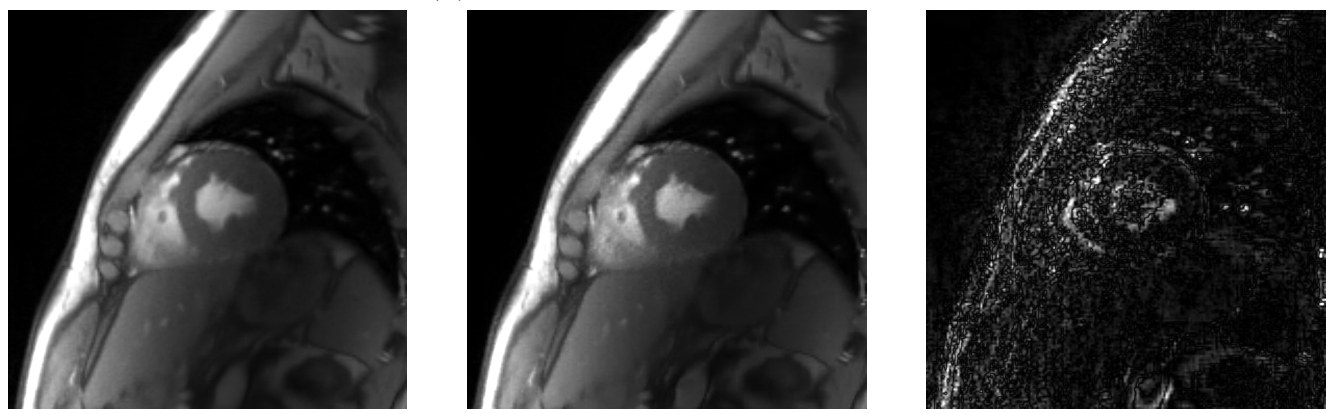

(c) end-systole end-exhalation
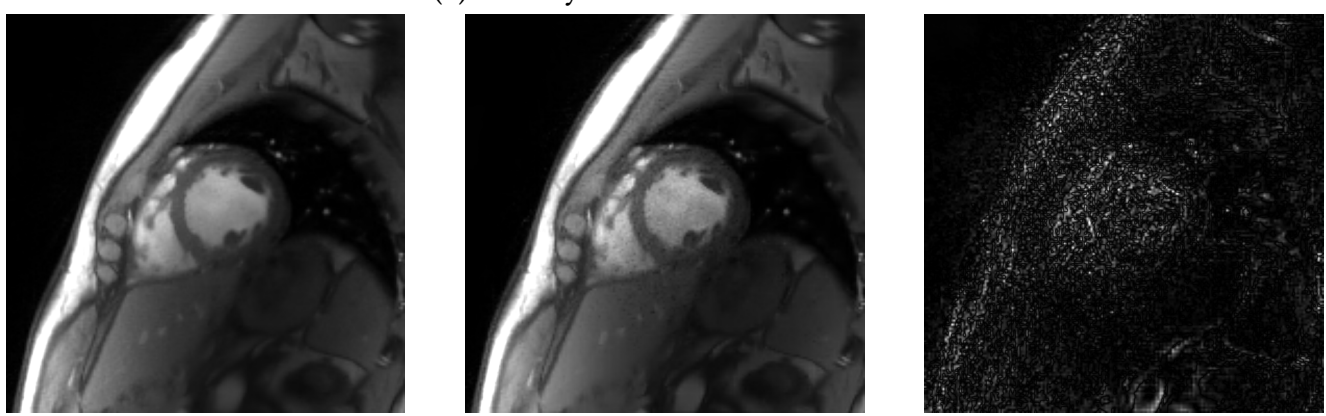

(d) end-diastole end-exhalation

Figure 5.12: SSA-FARY reconstruction with ESPIRiT (left) and with XD-NLINV (center) in different cardiac and respiratory states, together with their difference (right) scaled up by a factor of $\times 10$. Both approaches show excellent image quality, with very little difference. 


\subsection{Discussion}

The proposed method, XD-NLINV extends non-linear reconstruction to arbitrary dimensions while including compressed sensing This allows exploiting prior knowledge about the acquired data to achieve improved reconstruction. Because of the ease of adding arbitrary regularization, the bi-linear original problem was formulated as two linear subproblems, one for the coil sensitivity profiles and one for the images, which have to be solved in tandem. As the various reconstructions show, it is applicable to a wide array of imaging methods.

The results for the $\mathrm{DCE}$ dataset (Figure 5.1 - Figure 5.6 show an improved image quality compared to real-time NLINV Even though separation between image content and coil sensitivity profiles is not perfect (see Figure 5.6), XD-NLINV]s signal development (Figure 5.2 shows that this is not a problem in practice, even for quantitative analysis. The imperfect separation is most likely due to the problem formulation: only the added regularization, especially the enforced smoothness of the coil sensitivity profiles, discriminates between coil profiles and images. However, the post-processing described in Section 5.1 leads to proper final results, as the agreement between the real-time NLINV and the XD-NLINV reconstructions in Figure 5.2 shows.

The ESPIRiT+PICS reconstructions in Figure 5.10 and Figure 5.12 successfully used lower-dimensional coil profiles than the reconstructed images: in the former case, a single set of coil profiles was used for all frames, so the coil profiles were time-independent, while in the later case, the coil profiles differed for each respiratory state but were constant along cardiac state. This shows that the full dimensionality, as is currently done in XDNLINV is not always needed in the coil profiles. Therefore, in the future, a version of XD-NLINV which determines coil profiles of a lower dimensionality is conceivable and could reduce the problem size. It must be noted here that ESPIRiT coil profiles are always recalculated, while XD-NLINV estimates them during image reconstruction.

However, in cases where the full dimensionality is necessary, such as in the interactive real-time dataset shown in Figure 5.11 ESPIRiT+PICS leads to artifacts in signal loss. This is the case since the ESPIRiT calibration was done on the first part of the dataset and so was no longer accurate after the slice movements and rotations between Figure 5.11(a) and Figure 5.11(b) XD-NLINV in that case, calculates coil profiles for each time frame, and therefore manages correct reconstruction.

In conclusion, XD-NLINV has been shown to perform on par with current state-of-theart methods where they are applicable. Additionally, it produces high-quality images in cases where other techniques such as ESPIRiT are not applicable. Because of its flexibility, 
the possible applications of XD-NLINV are numerous. By lifting the limitation on specific data shapes and instead allowing full multi-dimensional reconstruction, XD-NLINV]can be adapted to many image setting in MRI 



\section{Banding-free Balanced Steady-State Free Precession}

\subsection{Introduction}

In this chapter, a third way to extend the dimensionality of the problem is explored: We will extend the coil dimension of the reconstruction to integrate parts of the measurements which have a different weighting due to physical phenomena.

We will explore an example for removal of banding artifacts in balanced steady-state free precession (bSSFP), which is of practical importance for cardiac imaging at high field strengths.

Initial results for brain imaging have been presented at the $26^{\text {th }}$ Annual Meeting of the International Society for Magnetic Resonance in Medicine [87].

Balanced steady-state free precession (bSSFP), described in more detail in Section 2.2.2 is a fast MRI sequence often used in clinical practice because of its high $\mathrm{SNR}$ and its unique $T_{2} / T_{1}$ contrast. However, it is sensitive to off-resonances which result in banding artifacts [26]. Figure 6.1 shows such an artifact in a cardiac bSSFP acquisition. As banding artifacts worsen with increased field strength, banding-free bSSFP is especially challenging at $3 \mathrm{~T}$ and beyond. For this reason, approaches for reducing the impact of banding artifacts have been developed, first among them $\mathrm{rf}$ phase cycling, which multiplies the required scan time. Joint reconstruction approaches, such as those introduced by Ilicak et al. [88] and Bilgic et al. [89], can decrease this extra scan time. Still, for each phase cycle (typically between 4 and 8), a new steady state needs to be prepared, requiring a recovery period between cycles and a setup period into each new steady state.

A different approach is dynamically phase-cycled or frequency-modulated steady-state free precession (fm-SSFP) 90 91], where the rf-phase is slightly changed in each excitation, leading to a time-varying steady state. These approaches often require complicated reconstruction methods which precisely model the fm-SSFP dynamic [92, 93].

Here, a new method is introduced which can be viewed as a combination of joint 
reconstruction of different phase cycles and fm-SSFP combining a simple extension of conventional NLINV reconstruction with a frequency-modulated acquisition. It exploits the fact that small changes in the rf phase increment do not strongly distort the signal response, as Foxall [90] could show by analysis of the bSSFP signal. In exchange for increasing the dimensionality of the reconstruction problem, it removes banding artifacts from bSSFPIMRI

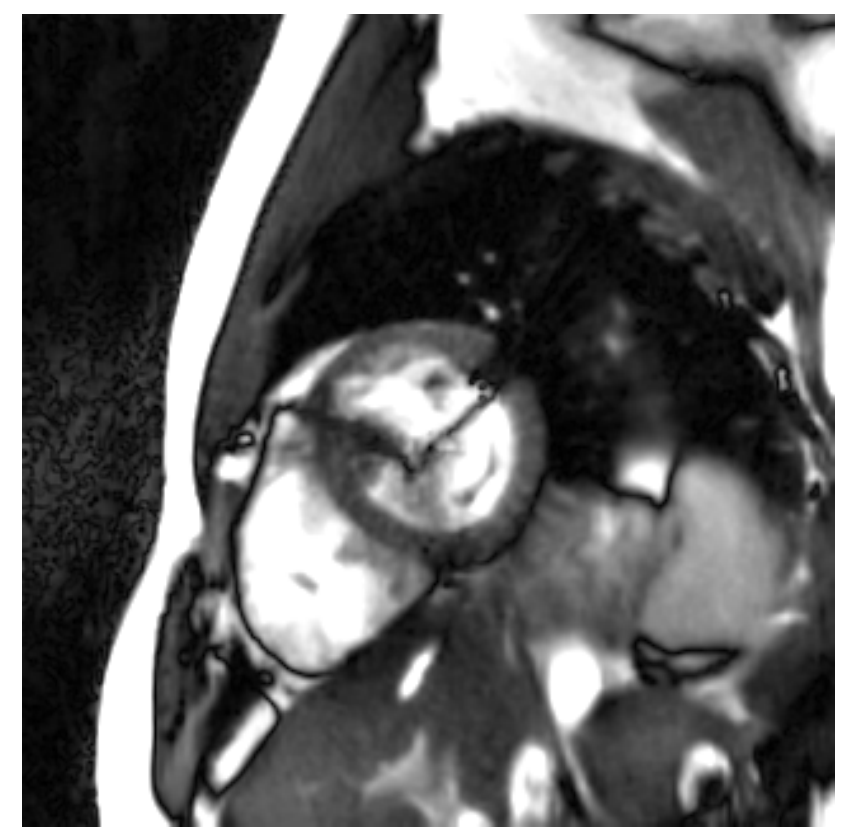

Figure 6.1: Selected frame of a short-axis cardiac bSSFP acquisition. A banding artifact passes through the left ventricle, rendering the image unusable.

\subsection{VC-NLINV}

This method, termed virtual-coil (VC) NLINV comprises changes in both acquisition and reconstruction compared to joint reconstruction of phase-cycled bSSFP and frequencymodulated bSSFP

In contrast to fm-SSFP where the rf phase is changed for each excitation, VC-NLINV acquires a number of radial spokes with the same $[\mathrm{rf}$ phase before changing it for the next frame. This is done so that each frame, instead of each excitation, can be regarded as a separate phase cycle.

These frames, each containing a different phase cycle, are then stacked in the coil dimension as additional virtual coils and reconstructed using the regularized non-linear inverse reconstruction (NLINV) This, in effect, constrains the single image to simultane- 
ously explain all phase cycles, while the banding artifacts are regarded as changes in the coil sensitivity profiles only.

\subsection{Methods}

I acquired bSSFP data of a transversal slice through the brain of a volunteer with no known illnesses. These data were acquired at $3 \mathrm{~T}$ using a 20 -channel head coil. Further sequence parameters were: TR/TE: $4.4 \mathrm{~ms} / 2.2 \mathrm{~ms}$, flip angle: $40^{\circ}$, FOV: $192 \times 192 \mathrm{~mm}^{2}$, resolution: $1 \times 1 \times 5 \mathrm{~mm}^{3}$, bandwidth per pixel: $840 \mathrm{~Hz}$. I acquired two datasets with differing phase increments and spokes per frame: dataset a) with $2^{\circ}$ phase increment and 43 spokes per frame, and dataset b) with $5^{\circ}$ phase increment and 17 spokes per frame. Each dataset covers phase cycles between $180^{\circ}$ and $360^{\circ}$, leading to acquisition times of $17 \mathrm{~s}$ for the 90 frames of dataset a) and $2.7 \mathrm{~s}$ for the 36 frames of dataset $b$ ).

Both datasets were then reconstructed twice: once with a real-time NLINV reconstruction, so that each individual phase cycle is reconstructed, and once using the proposed VC-NLINV Both reconstruction were done using the Berkeley Advanced Reconstruction Toolbox (BART) [37].

\subsection{Results}

The VC-NLINV reconstructions, together with selected phase cycles from the real-time reconstruction, are shown in Figure 6.2 for dataset a) and in Figure 6.3 for dataset b). In both cases, banding artifacts are visible in the individual phase cycles. Using VC-NLINV however, eliminates those banding artifacts.

Residual streak artifacts remain when using 17 spokes (Figure 6.3); no streak artifacts appear when using 43 spokes per frame (Figure 6.2).

Figure 6.4 and Figure 6.5 show signal curves of regions-of-interest in the white matter of the brain and in the cerebrospinal fluid (CSF) for both datasets. Here, we can see that for the dataset with 17 spokes and $5^{\circ}$ phase shift per frame, the signal development is noisy. Furthermore, for both datasets, the signal curves do not show $180^{\circ}$ periodicity.

\subsection{Discussion}

While the banding artifacts are removed in the VC-NLINV]reconstruction, the residual streak artifacts in Figure 6.3 remain. 


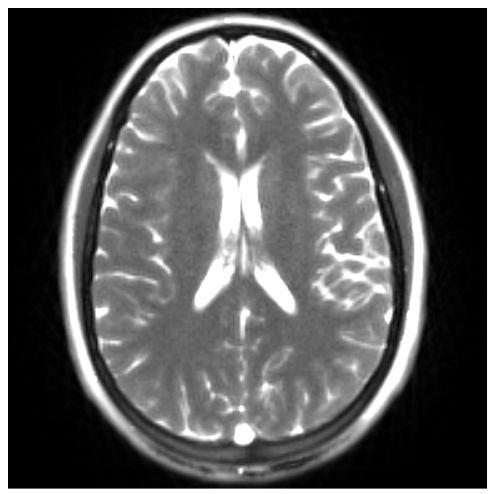

$210^{\circ}$

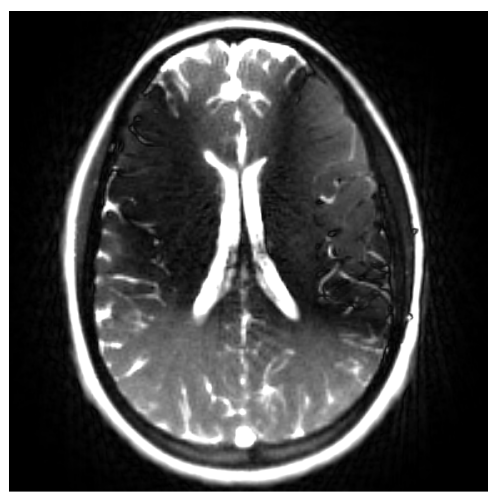

$290^{\circ}$

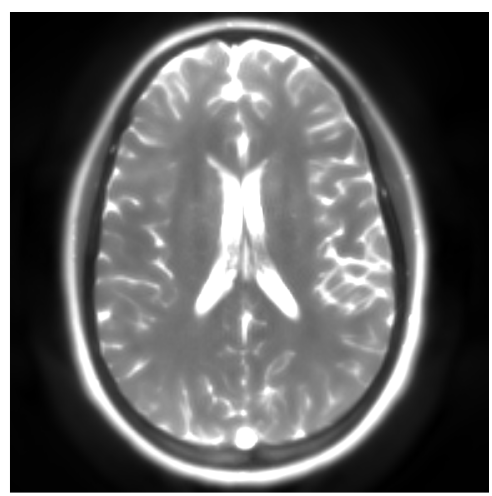

VC-NLINV

Figure 6.2: Two selected phase cycles of the real-time NLINV reconstruction of dataset a) (left and center) together with the reconstruction using the proposed VC-NLINV (right). The individual phase cycles show banding artifacts, which are eliminated using VC-NLINV.

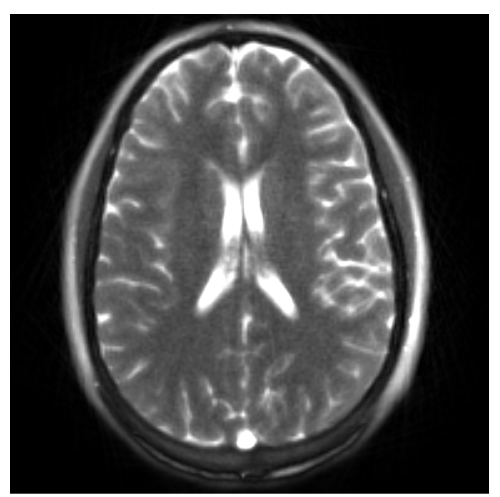

$210^{\circ}$

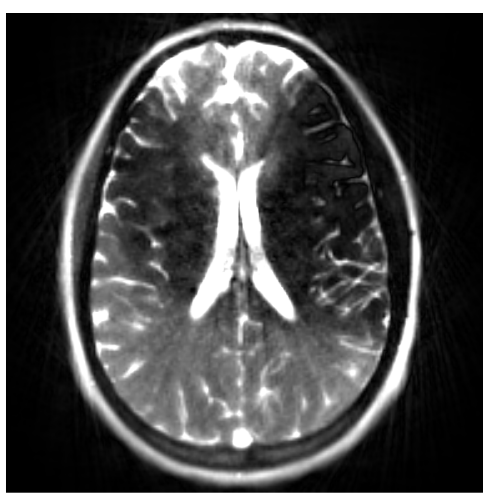

$290^{\circ}$

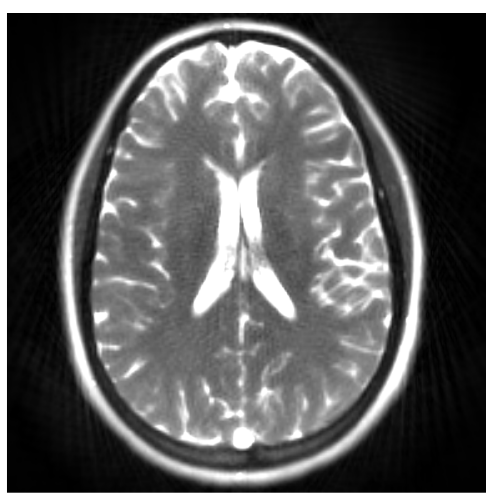

VC-NLINV

Figure 6.3: Two selected phase cycles of the real-time NLINV reconstruction of dataset b) (left and center) together with the reconstruction using the proposed VC-NLINV (right). Here, too, the individual phase cycles show banding artifacts, which are eliminated using VC-NLINV Additionally, this dataset contains streak artifacts in both the real-time NLINV and the VC-NLINV reconstruction. 

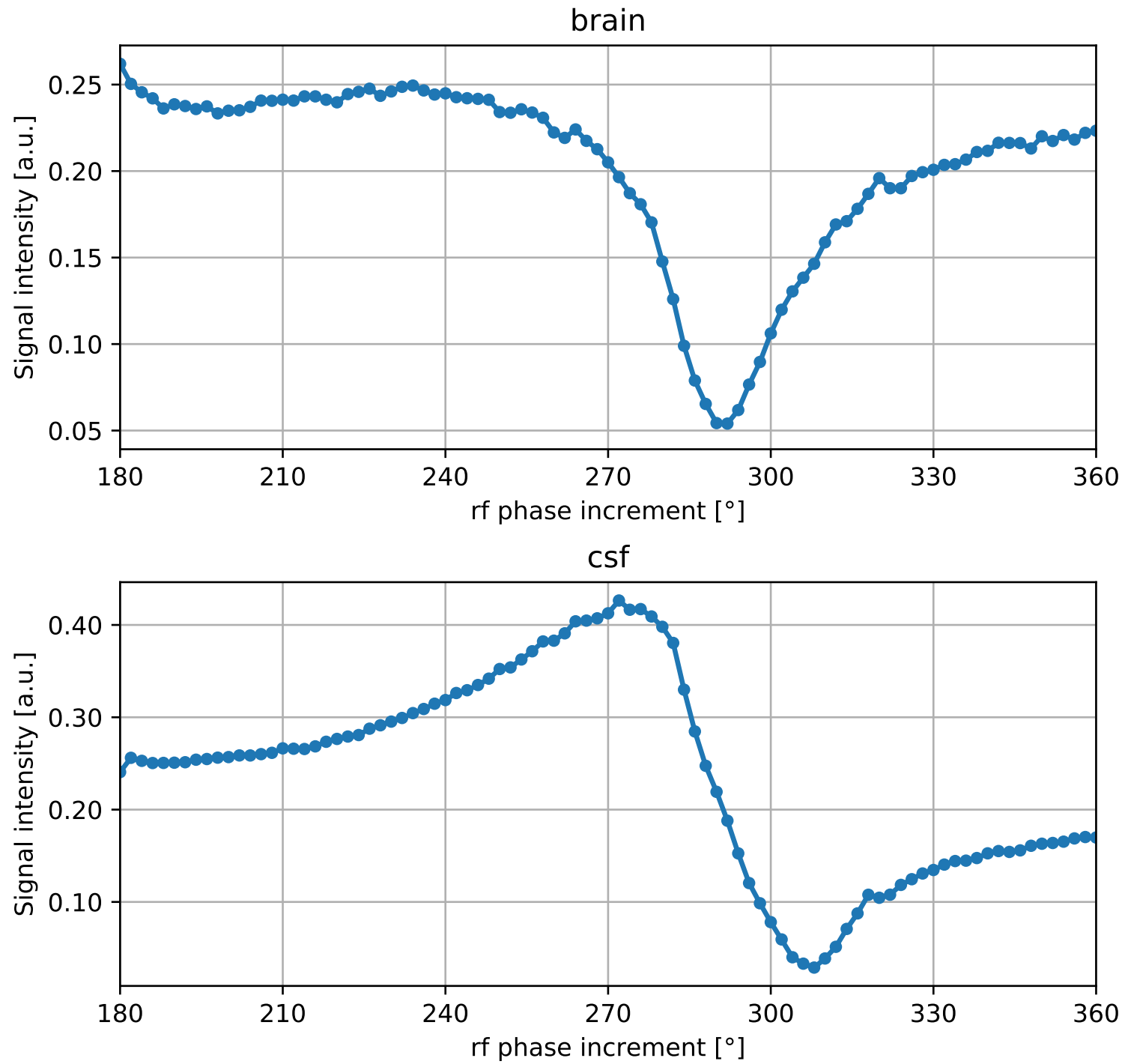

Figure 6.4: Signal development for each frame in regions-of-interest of the real-time reconstruction of dataset a). According to the theory presented in Section 2.2.2 both signal curves should ideally show $180^{\circ}$ symmetry, but both of them do not. 

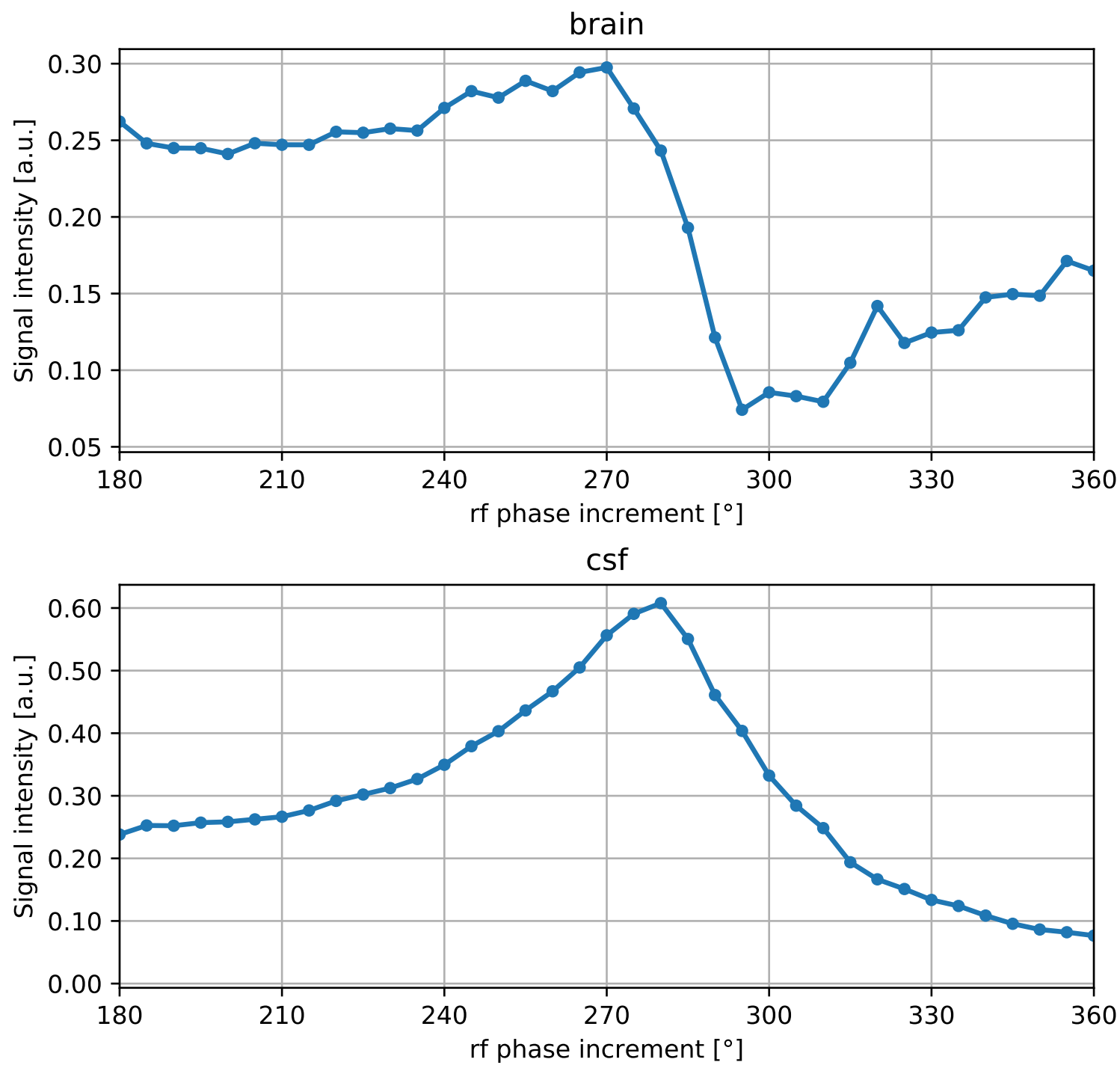

Figure 6.5: Signal development for each frame in regions-of-interest of the real-time reconstruction of dataset $b$ ). While the signal shows the same overall structure as Figure 6.4 it also shows more noise. Additionally, is also does not show the expected $180^{\circ}$ symmetry. 
According to Foxall [90], fm-SSFP with phase changes of over $3^{\circ}$ shows a distorted signal response compared to bSSFP Since this is undesirable in the present application, only small phase changes are used. Note however, that this limit of $3^{\circ}$ is for continual phase changes, while the rf phase is only changed for each frame in VC-NLINV so once every 17 or 43 excitations. This would also explain why the VC-NLINV reconstruction of the dataset with $5^{\circ}$ (Figure 6.3) does not show strong differences to the dataset with $2^{\circ}$ phase change per frame. However, the signal curves shown in Figure 6.4 and Figure 6.5 show deviations from the expected $180^{\circ}$ symmetry. This would indicate a distorted signal response even for $2^{\circ}$ phase shift every 43 excitations.

Small changes in the phase increment only slightly move the banding artifacts. Therefore, a much larger number of phase cycles must be combined when using VC-NLINV compared to traditional phase cycling. Still, by only slightly disturbing the steady state, the proposed scheme can acquire data continuously without recovery or preparation periods for each cycle.

The current implementation uses 5 sets of uniformly distributed radial spokes which repeat every 5 frames. Changing this to acquiring a new set of spokes in each frame, for example by using the golden angle between spokes, might improve image quality.

In conclusion, VC-NLINV, a high-dimensional extension of NLINV to include phase cycles as virtual coils, can be used to reconstruct banding free images from data acquired in less than $3 \mathrm{~s}$. However, the signal response needs further study to confirm that it is not unduly changed from regular bSSFP 



\section{Summary and Outlook}

In this thesis, I introduced several higher-dimensional extensions to the signal model of magnetic resonance imaging relating to artifact mitigation, coil sensitivity calculation, and improved reconstruction.

Chapter 4 introduced ENLIVE which relaxes the model by allowing multiple images and maps, enlarging the problem. This relaxed model can accommodate violations of the simple MRI signal model, thereby improving its robustness. ENLIVE was evaluated on datasets covering a number of MRI methods of current interest, including phase-constrained imaging, highly-undersampled acquisitions, along with examples using non-Cartesian and real-time MRI. In all of these cases and in comparisons with state-of-the-art methods, it provided robust reconstructions of high quality. In contrast to previously proposed robust reconstruction approaches which are based on the SVD it is computationally much more efficient making it suitable for applications where SVD-based methods are currently not feasible, such as large $3 \mathrm{D}$ reconstruction and real-time MRI.

Chapter 5 describes the extension of NLINV to data of arbitrary dimensionality. Apart from improving image quality by leveraging compressed sensing in addition to parallel imaging, this allows to exploit the correlations in additional dimensions such as space, time, cardiac state and respiratory state. XD-NLINV is designed as a generic tool for image reconstruction. Due to its self-calibrating nature it can be applied directly to various imaging scenarios and was shown to provide image quality on par with state-of-the-art reconstruction methods in dynamic contrast enhanced (DCE) MRI. virtual moving table, interactive real-time MRI and the novel self-gating approach SSA-FARY.

Chapter 6 discusses how different smoothly weighted images obtained from the measurement process can be jointly reconstructed as additional virtual channels. This concept is evaluated on a bSSFP acquisition commonly used in cardiac MRI. Here, together with a phase-cycled acquisition which provides differently weighted images, it can avoid banding artifacts completely, which are a common problem in clinical practice at higher field strengths.

The common scheme in all extensions is that increasing the dimensionality of the reconstruction allows the exploitation of correlations using regularization schemes without 


\section{Summary and Outlook}

assuming a specific signal behavior. This is shown to increase reliability of measurements while at the same time reducing the time needed for data acquisition. For image reconstruction, this comes at the cost of increased model size and problem complexity. Here, further optimization of the numerical implementation to make better use of the capabilities of modern high-performance parallel processing platforms is necessary. $\mathrm{Nu}$ merical algorithms which split the problem into smaller parts will also help to address this problem. The alternating minimization scheme used for XD-NLINV in this thesis can serve as a starting point for the development of such algorithms.

Since the formulation of XD-NLINV allows for easy addition of arbitrary regularization, novel methods such as infimal convolution of total-generalized variation [94] could be added in the future. Additionally, methods restricting the rank of the reconstruction, as explored in Ong [95] would be of high interest and could be integrated as well.

In conclusion, this thesis introduced several extensions to regularized non-linear inverse reconstruction (NLINV) showing how such extension benefit the reliability, robustness, and quality of MRI Integrating calibrationless parallel imaging, compressed sensing, and joint reconstruction techniques in a unified framework, these extensions cover a large range of state-of-the art image reconstruction techniques and open up new opportunities, specifically in cardiac imaging. 


\section{A. Regularization parameters}

In Table A.1 the regularization parameters for the ESPIRiT+PICS and XD-NLINV the reconstructions in this thesis are collected. The vastly different scaling between XDNLINV and ESPIRiT+PICS is due to an implementation detail: In the XD-NLINV the raw data is scaled to 100 times the sum of the sizes of the extra dimensions (that includes all dimensions apart from spatial dimensions and the coil profiles, in particular it includes time and cardiac and respiratory phase). So for proper compatibility of the data scaling and the regularization, this extra scaling should also be applied to the regularization parameters, and indeed this will be the case in the future. Note that this scaling is only done for the reconstruction, it is removed afterwards.

Table A.1: Regularization parameters used in Chapter 5

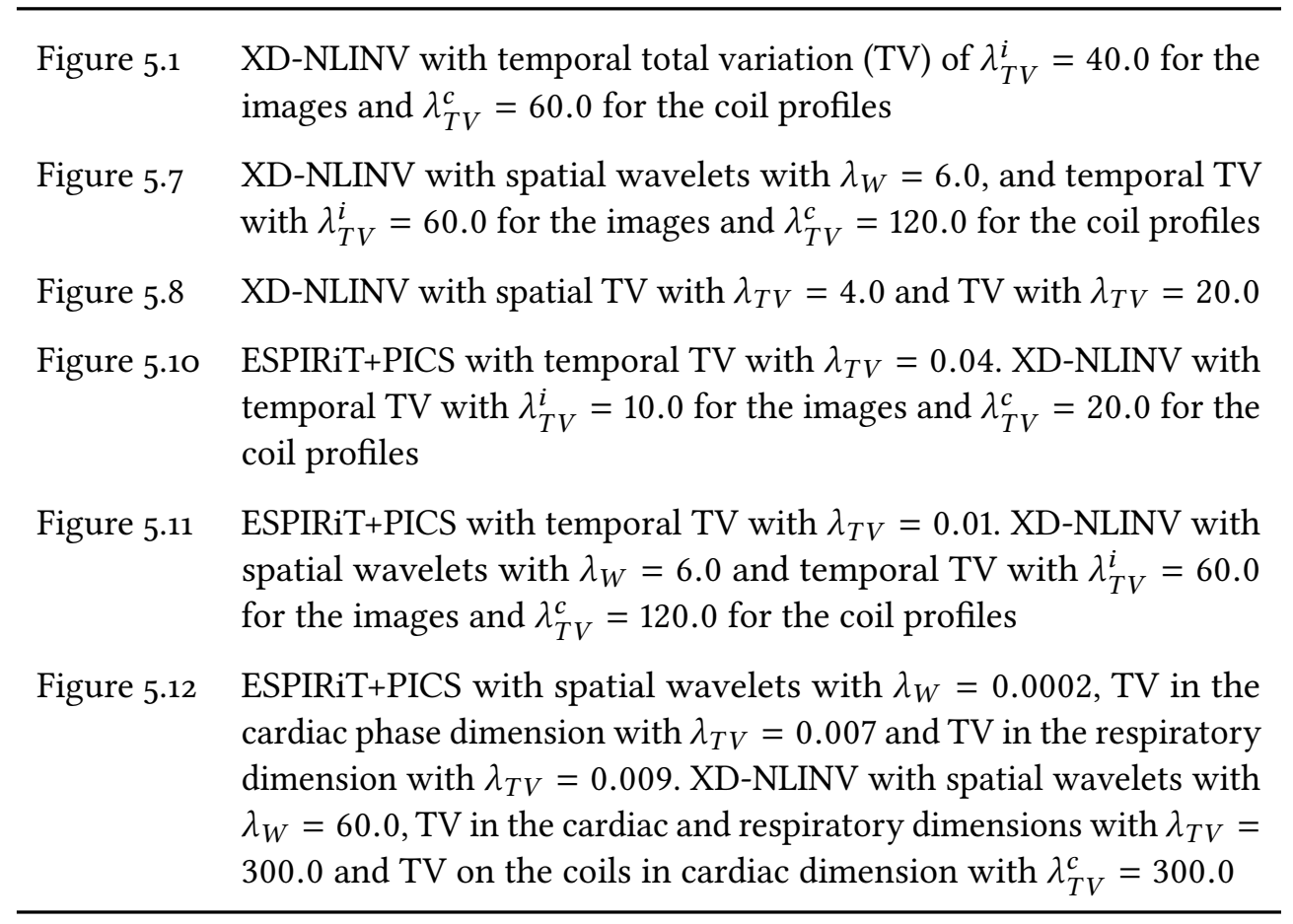





\section{Bibliography}

[1] S. Tokur, K. Lederle, D. Terris, M. Jarczok, S. Bender, S. Schoenberg, and G. Weisser. "Process analysis to reduce MRI access time at a German University Hospital". Int J Qual Health C 24 (2011), pp. 95-99. DO I:10.1093/intqhc/mzr077 (cit. on p.11.

[2] J. Frahm, A. Haase, D. Matthaei, W. Haenicke, and K. D. Merboldt. "HochfrequenzImpuls und Gradienten-Impuls-Verfahren zur Aufnahme von schnellen NMRTomogrammen unter Benutzung von Gradientenechos”. German pat. req. Max Planck Gesellschaft. 1985-O2 (cit. on pp. 囵11).

[3] A. Haase, J. Frahm, D. Matthaei, W. Hanicke, and K.-D. Merboldt. "FLASH imaging. Rapid NMR imaging using low flip-angle pulses”. F. Magn. Reson. 67 (1986), pp. 258266. DOI: $10.1016 / 0022-2364(86) 90433-6$ (cit. on p. 11).

[4] J. Frahm, A. Haase, and D. Matthaei. "Rapid NMR imaging of dynamic processes using the FLASH technique”. Magn. Reson. Med. 3 (1986), pp. 321-327 (cit. on p. 11).

[5] D. K. Sodickson and W. J. Manning. "Simultaneous acquisition of spatial harmonics (SMASH): fast imaging with radiofrequency coil arrays”. Magn. Reson. Med. 38 (1997), pp. 591-603 (cit. on p. 1).

[6] K. P. Pruessmann, M. Weiger, M. B. Scheidegger, and P. Boesiger. "SENSE: sensitivity encoding for fast MRI”. Magn. Reson. Med. 42 (1999), pp. 952-962 (cit. on pp. 1 . 11 21.

[7] M. A. Griswold, P. M. Jakob, R. M. Heidemann, M. Nittka, V. Jellus, J. Wang, B. Kiefer, and A. Haase. "Generalized autocalibrating partially parallel acquisitions (GRAPPA)”. Magn. Reson. Med. 47 (2002), pp. 1202-1210 (cit. on pp.111.21).

[8] M. Uecker, S. Zhang, D. Voit, A. Karaus, K.-D. Merboldt, and J. Frahm. "Real-time MRI at a resolution of 20 ms”. NMR Biomed. 23 (2010), pp. 986-994. DO I:10.1002/ nbm.1585 (cit. on pp. 1, 15, 53, 54).

[9] M. Uecker, T. Hohage, K. T. Block, and J. Frahm. "Image reconstruction by regularized nonlinear inversion-joint estimation of coil sensitivities and image content". Magn. Reson. Med. 6o (2008), pp. 674-682 (cit. on pp. 11 11, 14, 21, 22, 24). 
[10] K. T. Block, M. Uecker, and J. Frahm. "Undersampled radial MRI with multiple coils. Iterative image reconstruction using a total variation constraint”. Magn. Reson. Med. 57 (2007), pp. 1086-1098. D o I:10.1002/mrm.21236 (cit. on pp. 2. 16).

[11] M. Lustig, D. Donoho, and J. M. Pauly. "Sparse MRI: The application of compressed sensing for rapid MR imaging”. Magn. Reson. Med. 58 (2007), pp. 1182-1195 (cit. on pp. 2, 16.

[12] K. T. Block, M. Uecker, and J. Frahm. "Model-Based Iterative Reconstruction for Radial Fast Spin-Echo MRI”. IEEE Trans. Med. Imaging 28 (2009), pp. 1759-1769. DOI: $10.1109 /$ tmi.2009.2023119 (cit. on p. 2).

[13] T. J. Sumpf, M. Uecker, S. Boretius, and J. Frahm. "Model-based nonlinear inverse reconstruction for T2-mapping using highly undersampled spin-echo MRI". $\mathcal{F}$. Magn. Reson. Imaging 34 (2011), pp. 420-428. Do I: 10.1002/jmri.22634 (cit. on p.2.

[14] M. Doneva, P. Börnert, H. Eggers, C. Stehning, J. Sénégas, and A. Mertins. "Compressed sensing reconstruction for magnetic resonance parameter mapping”. Magn. Reson. Med. 64 (2010), pp. 1114-1120. DO I:10.1002/mrm.22483 (cit. on p. 2).

[15] M. Uecker, P. Lai, M. J. Murphy, P. Virtue, M. Elad, J. M. Pauly, S. S. Vasanawala, and M. Lustig. "ESPIRiT-an eigenvalue approach to autocalibrating parallel MRI: where SENSE meets GRAPPA". Magn. Reson. Med. 71 (2014), pp. 990-1001 (cit. on pp. 2, 21, 23, 37, 40, 44.

[16] H. C. M. Holme. "Preparatory data analysis for the reconstruction of real-time MRI data”. Master's Thesis. Georg-August-Universität Göttingen, 2016. arXiv: 1906.05125 [physics.med-ph] (cit. on p. 5).

[17] E. M. Haacke, R. W. Brown, M. R. Thompson, and R. Venkatesan. Magnetic Resonance Imaging: Physical Principles and Sequence Design. Wiley, 1999. Is B N: 9780471351283 (cit. on pp. 5, 7).

[18] M. A. Bernstein, K. F. King, and X. J. Zhou. Handbook of MRI Pulse Sequences. Elsevier Science, 2004. I S B N: 9780080533124 (cit. on pp. 5 , 12).

[19] E. M. Haacke, Z. P. Liang, and S. H. Izen. "Superresolution reconstruction through object modeling and parameter estimation". IEEE Trans. Acoust., Speech, Signal Processing 37 (1989), pp. 592-595. D O I: 10.1109/29.17545(cit. on pp. 5 37).

[20] F. Bloch. "Nuclear Induction". Phys. Rev. 70 (1946), pp. 460-474. u R L:http://link aps.org/doi/10.1103/PhysRev.70.460 (cit. on p. 7). 
[21] M. Uecker. "Nonlinear Reconstruction Methods for Parallel Magnetic Resonance Imaging”. PhD thesis. Georg-August-Universität Göttingen, 2009 (cit. on pp. 8, 10 14. 15.

[22] K. T. Block. "Advanced methods for radial data sampling in magnetic resonance imaging”. PhD thesis. Georg August Universität Göttingen, 2008 (cit. on p. 9).

[23] E. G. Larsson, D. Erdogmus, R. Yan, J. C. Principe, and J. R. Fitzsimmons. "SNRoptimality of sum-of-squares reconstruction for phased-array magnetic resonance imaging”. F. Magn. Reson. 163 (2003), pp. 121-123. D O I:10.1016/S1090-7807(03) $00132-0$ (cit. on p. 11).

[24] V. Roeloffs, D. Voit, and J. Frahm. "Spoiling without additional gradients: Radial FLASH MRI with randomized radiofrequency phases". Magn. Reson. Med. 75 (2016), pp. 2094-2099. DOI: $10.1002 / \mathrm{mrm} .25809$ (cit. on pp. 12,56 .

[25] H. Y. Carr. "Steady-State Free Precession in Nuclear Magnetic Resonance”. Phys. Rev. 112 (5 1958), pp. 1693-1701. DOI: 10.1103/PhysRev.112.1693 uRL: https: //link.aps.org/doi/10.1103/PhysRev.112.1693 (cit. on p.12).

[26] K. Scheffler and S. Lehnhardt. "Principles and applications of balanced SSFP techniques". Eur. Radiol. 13 (2003), pp. 2409-2418. Do I:10.1007/s00330-003-1957-x (cit. on pp. 12, 75).

[27] N. Scholand. "Model-Based Reconstruction for Quantitative MRI using the Bloch Equations”. Master's Thesis. Georg-August-Universität Göttingen, 2019. arXiv: 1905.03188 [physics.med-ph] (cit. on p. 13.

[28] K. P. Pruessmann, M. Weiger, P. Boernert, and P. Boesiger. "Advances in sensitivity encoding with arbitrary k-space trajectories”. Magn. Reson. Med. 46 (2001), pp. 638651 (cit. on p. 14).

[29] L. Ying and J. Sheng. "Joint image reconstruction and sensitivity estimation in SENSE (JSENSE)”. Magn. Reson. Med. 57 (2007), pp. 1196-1202 (cit. on pp. 14, 21, 55).

[30] M. Uecker, S. Zhang, and J. Frahm. "Nonlinear inverse reconstruction for real-time MRI of the human heart using undersampled radial FLASH”. Magn. Reson. Med. 63 (2010), pp. 1456-1462. D OI: $10.1002 / \mathrm{mrm} .22453$ (cit. on pp. $15,41,48$, 54 .

[31] R. Chartrand. "Exact Reconstruction of Sparse Signals via Nonconvex Minimization”. IEEE Signal Processing Lett. 14 (2007), pp. 707-710. D O I:10.1109/LSP.2007 898300 (cit. on p. 16). 
[32] A. Beck and M. Teboulle. "A Fast Iterative Shrinkage-Thresholding Algorithm for Linear Inverse Problems”. SIAM f. Img. Sci. 2 (2009), pp. 183-202. D o I:10.1137/ 080716542 (cit. on pp. 16 55.

[33] M. V. Afonso, J. M. Bioucas-Dias, and M. A. T. Figueiredo. "An Augmented Lagrangian Approach to the Constrained Optimization Formulation of Imaging Inverse Problems". Trans. Img. Proc. 20 (2011), pp. 681-695. Do 1: $10.1109 /$ TIP 2010.2076294 (cit. on pp. 16 . 55 .

[34] J. Eckstein and D. P. Bertsekas. "On the Douglas-Rachford splitting method and the proximal point algorithm for maximal monotone operators”. Math. Program. 55 (1992), pp. 293-318. D oi: 10.1007/BF01581204 (cit. on pp. 16 55).

[35] M. Lustig, S. J. Kim, and J. M. Pauly. "A fast method for designing time-optimal gradient waveforms for arbitrary k-space trajectories". IEEE Trans. Med. Imag. 27 (2008), pp. 866-873 (cit. on p. 16).

[36] S. Vasanawala, M. Murphy, M. Alley, P. Lai, K. Keutzer, J. Pauly, and M. Lustig. "Practical parallel imaging compressed sensing MRI: Summary of two years of experience in accelerating body MRI of pediatric patients”. Proc. IEEE Int. Symp. Biomed. Imaging. Chicago: IEEE, 2011, pp. 1039-1043 (cit. on pp. 16, 41).

[37] M. Uecker, F. Ong, J. I. Tamir, D. Bahri, P. Virtue, J. Y. Cheng, T. Zhang, and M. Lustig. "Berkeley advanced reconstruction toolbox". Proc. Intl. Soc. Mag. Reson. Med. Vol. 23. Toronto, 2015, p. 2486 (cit. on pp. 17. 39, 77).

[38] H. C. M. Holme, S. Rosenzweig, F. Ong, R. N. Wilke, M. Lustig, and M. Uecker. "ENLIVE: An Efficient Nonlinear Method for Calibrationless and Robust Parallel Imaging”. Sci. Rep. 9 (2019), p. 3034. DO I:10.1038/s41598-019-39888-7 (cit. on p. 19.

[39] P. J. Shin, P. E. Z. Larson, M. A. Ohliger, M. Elad, J. M. Pauly, D. B. Vigneron, and M. Lustig. "Calibrationless parallel imaging reconstruction based on structured low-rank matrix completion”. Magn. Reson. Med. 72 (2014), pp. 959-970. Do I: 10.1002/mrm.24997 (cit. on pp. 21, 37, 44).

[40] J. P. Haldar. "Low-Rank Modeling of Local k-Space Neighborhoods (LORAKS) for Constrained MRI”. IEEE Trans. Med. Imag. 33 (2014), pp. 668-681. Do I:10.1109/ TMI.2013.2293974 (cit. on pp. 21, 36. 
[41] J. P. Haldar and J. Zhuo. "P-LORAKS: Low-rank modeling of local k-space neighborhoods with parallel imaging data”. Magn. Reson. Med. 75 (2016), pp. 1499-1514. DO I: $10.1002 / \mathrm{mrm} .25717$ (cit. on pp. 21, 36).

[42] J. D. Trzasko and A. Manduca. "Calibrationless parallel MRI using CLEAR". Conf. Rec. Asilomar Conf. Signals Syst. Comput. Vol. 45. Pacific Grove, 2011, pp. 75-79 (cit. on pp. 21, 36.

[43] M. A. Griswold, S. Kannengiesser, R. M. Heidemann, J. Wang, and P. M. Jakob. "Fieldof-view limitations in parallel imaging”. Magn. Reson. Med. 52 (2004), pp. 1118-1126. DO I: $10.1002 / \mathrm{mrm} .20249$ (cit. on p.21).

[44] M. Uecker and M. Lustig. "Estimating absolute-phase maps using ESPIRiT and virtual conjugate coils". Magn. Reson. Med. 77 (2017), pp. 1201-1207. D O I:10.1002/ mrm.26191 (cit. on pp. 21 40.

[45] H. C. M. Holme, F. Ong, S. Rosenzweig, R. N. Wilke, M. Lustig, and M. Uecker. "ENLIVE: A Non-Linear Calibrationless Method for Parallel Imaging using a LowRank Constraint”. Proc. Intl. Soc. Mag. Reson. Med. Vol. 25. Honolulu, 2017, p. 5160. U R L: ht tp: // indexsmart . mirasmart. com/ISMRM2017/PDFfiles/5160.html (cit. on p.22.

[46] D. Kundur and D. Hatzinakos. "Blind image deconvolution". IEEE Signal Process. Mag. 13 (1996), pp. 43-64. DOI:10.1109/79.489268 (cit. on p. 23).

[47] A. Ahmed, B. Recht, and J. Romberg. "Blind Deconvolution Using Convex Programming”. IEEE Trans. Inform. Theory 6o (2014), pp. 1711-1732 (cit. on p. 23).

[48] M. A. Davenport and J. Romberg. "An Overview of Low-Rank Matrix Recovery From Incomplete Observations”. IEEE f. Sel. Top. Signa. 10 (2016), pp. 608-622. D o I: 10.1109/JSTSP.2016.2539100 (cit. on pp. 23 37).

[49] B. Recht, M. Fazel, and P. A. Parrilo. "Guaranteed Minimum-Rank Solutions of Linear Matrix Equations via Nuclear Norm Minimization”. SIAM Review 52 (2010), pp. 471-501 (cit. on pp. 23 37).

[5o] P. Kovesi. Good Colour Maps: How to Design Them. 2015. arXiv:1509.03700 [cs.GR] (cit. on p. 27).

[51] K. H. Jin, D. Lee, and J. C. Ye. "A General Framework for Compressed Sensing and Parallel MRI Using Annihilating Filter Based Low-Rank Hankel Matrix". IEEE Trans. Comput. Imag. 2 (2016), pp. 480-495. DO I: $10.1109 /$ TCI . 2016.2601296 (cit. on p. 37). 
[52] D. Lee, K. H. Jin, E. Y. Kim, S.-H. Park, and J. C. Ye. "Acceleration of MR parameter mapping using annihilating filter-based low rank hankel matrix (ALOHA)". Magn. Reson. Med. 76 (2016), pp. 1848-1864. D O I: 10.1002/mrm. 26081 (cit. on p. 37).

[53] G. Ongie and M. Jacob. "A Fast Algorithm for Convolutional Structured LowRank Matrix Recovery”. IEEE Trans. Comput. Imag. 3 (2017), pp. 535-550. DO I: 10.1109/TCI.2017.2721819 (cit. on p. 37).

[54] G. Ongie and M. Jacob. "Off-the-Grid Recovery of Piecewise Constant Images from Few Fourier Samples”. SIAM J. Imag. Sci. 9 (2016), pp. 1004-1041. Do I:10.1137/ 15M1042280 (cit. on p. 37).

[55] M. Uecker, K. T. Block, and J. Frahm. "Nonlinear Inversion with L1-Wavelet Regularization - Application to Autocalibrated Parallel Imaging”. Proc. Intl. Soc. Mag. Reson. Med. Vol. 16. Toronto, 2008, p. 1479 (cit. on p. 37).

[56] S. G. Lingala and M. Jacob. "Blind Compressive Sensing Dynamic MRI”. IEEE Trans. Med. Imag. 32 (2013), pp. 1132-1145. D O I:10.1109/TMI.2013.2255133 (cit. on p. 37).

[57] J. P. Haldar and Z. P. Liang. "Spatiotemporal imaging with partially separable functions: A matrix recovery approach". Proc. IEEE Int. Symp. Biomed. Imaging. Rotterdam, 2010, pp. 716-719. D O I: 10.1109/ISBI.2010.5490076 (cit. on p. 37).

[58] M. Uecker and M. Lustig. "Making SENSE of Chemical Shift: Separating Species in Single-Shot EPI using Multiple Coils”. Proc. Intl. Soc. Mag. Reson. Med. Vol. 20. Melbourne, 2012, p. 2490 (cit. on p. 37).

[59] P. J. Shin et al. "Chemical Shift Separation with Controlled Aliasing for Hyperpolarized ${ }_{13}$ C Metabolic Imaging”. Magn. Reson. Med. 74 (2015), pp. 978-989 (cit. on p. 37.

[6o] S. J. Inati, M. S. Hansen, and P. Kellman. "A solution to the phase problem in adaptive coil combination”. Proc. Intl. Soc. Mag. Reson. Med. Vol. 21. Salt Lake City, 2013, p. 2627 (cit. on pp. 38,40 .

[61] B. Bilgic, J. P. Marques, L. L. Wald, and K. Setsompop. "Block Coil Compression for Virtual Body Coil without Phase Singularities”. Fourth International Workshop on MRI Phase Contrast \& Quantitative Susceptibility Mapping. Graz, 2016. U RL: https: //www . martinos .org/ berkin/2016_07_30_BCC_abstract . pdf (cit. on pp. 38.40 .

[62] D. O. Walsh, A. F. Gmitro, and M. W. Marcellin. "Adaptive reconstruction of phased array MR imagery". Magn. Reson. Med. 43 (2000), pp. 682-69o (cit. on p. 38). 
[63] M. Uecker and M. Lustig. "Memory-Saving Iterative Reconstruction on Overlapping Blocks of K-Space.” Proc. Intl. Soc. Mag. Reson. Med. Vol. 21. Salt Lake City, 2013, p. 2645 (cit. on p. 38).

[64] N. Li, W.-T. Wang, D. L. Pham, and J. A. Butman. "Artifactual microhemorrhage generated by susceptibility weighted image processing”. F. Magn. Reson. Imaging 41 (2015), pp. 1695-1700. DOI:10.1002/jmri.24728 (cit. on p. 38).

[65] X. Wang, V. Roeloffs, J. Klosowski, Z. Tan, D. Voit, M. Uecker, and J. Frahm. "Modelbased T1 mapping with sparsity constraints using single-shot inversion-recovery radial FLASH”. Magn. Reson. Med. 79 (2018), pp. 730-740. D o I: $10.1002 / \mathrm{mrm} .26726$ (cit. on pp. 38 40.

[66] O. Tange. "GNU Parallel - The Command-Line Power Tool”. ;login: The USENIX Magazine 36 (2011), pp. 42-47. Do I:10.5281/zenodo.16303, U R L: http://www.gnu org/s/parallel (cit. on p. 39).

[67] J. D. Willig-Onwuachi, E. N. Yeh, A. K. Grant, M. A. Ohliger, C. A. McKenzie, and D. K. Sodickson. "Phase-constrained parallel MR image reconstruction”. f Magn Reson 176 (2005), pp. 187-198 (cit. on p. 40.

[68] M. Blaimer, M. Gutberlet, P. Kellman, F. A. Breuer, H. Köstler, and M. A. Griswold. "Virtual coil concept for improved parallel MRI employing conjugate symmetric signals." Magn. Reson. Med. 61 (2009), pp. 93-102 (cit. on p. 40).

[69] M. Blaimer, M. Heim, D. Neumann, P. M. Jakob, S. Kannengiesser, and F. A. Breuer. "Comparison of phase-constrained parallel MRI approaches: Analogies and differences.” Magn. Reson. Med. 75 (2016), pp. 1086-1099 (cit. on p. 40.

[7o] A. Moussavi, M. Untenberger, M. Uecker, and J. Frahm. "Correction of gradientinduced phase errors in radial MRI". Magn. Reson. Med. 71 (2013), pp. 308-312. Do I: 10.1002/mrm. 24643 (cit. on p. 41).

[71] J. Hennig, A. Nauerth, and H. Friedburg. "RARE imaging: a fast imaging method for clinical MR”. Magn. Reson. Med. 3 (1986), pp. 823-833 (cit. on p. 41).

[72] F. Ong, S. Amin, S. Vasanawala, and M. Lustig. "mridata.org: An Open Archive for Sharing MRI Raw Data”. Proc. Intl. Soc. Mag. Reson. Med. Vol. 26. Paris, 2018. U R L: http://indexsmart .mirasmart. com/ISMRM2018/PDFfiles/3425.html (cit. on p. 41. 
[73] F. A. Breuer, M. Blaimer, M. F. Mueller, N. Seiberlich, R. M. Heidemann, M. A. Griswold, and P. M. Jakob. "Controlled aliasing in volumetric parallel imaging (2D CAIPIRINHA)”. Magn. Reson. Med.55 (2006), pp. 549-556. D o I: $10.1002 / \mathrm{mrm} .20787$ (cit. on p. 41).

[74] V. Roeloffs, X. Wang, T. J. Sumpf, M. Untenberger, D. Voit, and J. Frahm. "Modelbased reconstruction for T1 mapping using single-shot inversion-recovery radial FLASH”. Int. F. Imag. Syst. Tech. 26 (2016), pp. 254-263. D I: 10.1002/ima.22196 (cit. on p. 44).

[75] X. Wang, A. A. Joseph, O. Kalentev, K.-D. Merboldt, D. Voit, V. B. Roeloffs, M. van Zalk, and J. Frahm. "High-resolution myocardial T1 mapping using single-shot inversion recovery fast low-angle shot MRI with radial undersampling and iterative reconstruction”. Brit. F. Radiol. 89 (2016), p. 20160255. Do I: $10.1259 /$ bjr. 20160255 (cit. on p. 44).

[76] S. Rosenzweig, N. Scholand, H. C. M. Holme, and M. Uecker. Cardiac and Respiratory Self-Gating in Radial MRI using an Adapted Singular Spectrum Analysis (SSA-FARI). 2018. arXiv: 1812.09057 [physics.med-ph] (cit. on pp. 45 54 57).

[77] S. Zhang, M. Uecker, D. Voit, K. Merboldt, and J. Frahm. "Real-time cardiovascular magnetic resonance at high temporal resolution: radial FLASH with nonlinear inverse reconstruction”. F. Cardiov. Magn. Reson. 12 (2010), p. 39 (cit. on p. 48).

[78] D. Voit, S. Zhang, C. Unterberg-Buchwald, J. M. Sohns, J. Lotz, and J. Frahm. "Realtime cardiovascular magnetic resonance at $1.5 \mathrm{~T}$ using balanced SSFP and $40 \mathrm{~ms}$ resolution”. F. Cardiov. Magn. Reson. 15 (2013), pp. 1-8. D o I: $10.1186 / 1532-429 X$ 15-79 (cit. on p. 48).

[79] J. M. Sohns, J. T. Kowallick, A. A. Joseph, K. D. Merboldt, D. Voit, M. Fasshauer, W. Staab, J. Frahm, J. Lotz, and C. Unterberg-Buchwald. "Peak flow velocities in the ascending aorta-real-time phase-contrast magnetic resonance imaging vs. cine magnetic resonance imaging and echocardiography”. Quant. Imaging Med. Surg. 5 (2015), pp. 685- (cit. on p. 48.

[8o] A. Joseph, J. T. Kowallick, K.-D. Merboldt, D. Voit, S. Schaetz, S. Zhang, J. M. Sohns, J. Lotz, and J. Frahm. "Real-time flow MRI of the aorta at a resolution of $40 \mathrm{msec}$ ". f. Magn. Reson. Imaging 40 (2014), pp. 206-213. DOI:10.1002/jmri.24328 (cit. on p. 48. 
[81] C. Unterberg-Buchwald, C. O. Ritter, V. Reupke, R. N. Wilke, C. Stadelmann, M. Steinmetz, A. Schuster, G. Hasenfuß, J. Lotz, and M. Uecker. "Targeted endomyocardial biopsy guided by real-time cardiovascular magnetic resonance”. F. Cardiov. Magn. Reson. 19 (2017), p. 45. DOI:10.1186/s12968-017-0357-3 (cit. on p. 48).

[82] A. Seif Amir Hosseini et al. "Real-time MRI for the dynamic assessment of fundoplication failure in patients with gastroesophageal reflux disease”. Eur. Radiol. (2019). DO I: $10.1007 / \mathrm{s} 00330-019-06025-\mathrm{x}$ (cit. on p. 48).

[83] S. Rosenzweig, H. C. M. Holme, R. N. Wilke, D. Voit, J. Frahm, and M. Uecker. "Simultaneous multi-slice MRI using cartesian and radial FLASH and regularized nonlinear inversion: SMS-NLINV”. Magn. Reson. Med. 79 (2018), pp. 2057-2066. DO I: $10.1002 / \mathrm{mrm} .26878$ (cit. on p. 48 ).

[84] L. Feng, L. Axel, H. Chandarana, K. T. Block, D. K. Sodickson, and R. Otazo. "XDGRASP: Golden-angle radial MRI with reconstruction of extra motion-state dimensions using compressed sensing”. Magn. Reson. Med. 75 (2016), pp. 775-788. Do I: 10.1002/mrm.25665 (cit. on p. 54).

[85] J. Y. Cheng, T. Zhang, M. T. Alley, M. Uecker, M. Lustig, J. M. Pauly, and S. S. Vasanawala. "Comprehensive Multi-Dimensional MRI for the Simultaneous Assessment of Cardiopulmonary Anatomy and Physiology". Sci. Rep. 7 (2017), p. 5330 (cit. on p. 54).

[86] M. R. Hestenes and E. Stiefel. "Methods of conjugate gradients for solving linear systems”. J. Res. Nat. Bur. Stand. 49 (1952) (cit. on p. 55).

[87] H. C. M. Holme, N. Scholand, S. Rosenzweig, and M. Uecker. "Banding-Free Reconstruction in Frequency-Modulated bSSFP using Virtual Coils with Regularized Non-Linear Inversion”. Proc. Intl. Soc. Mag. Reson. Med. Vol. 26. Paris, 2018, p. 2870. URL: http://indexsmart.mirasmart. com/ISMRM2018/PDFfiles/2870.html (cit. on p. 75.

[88] E. Ilicak, L. K. Senel, E. Biyik, and T. Çukur. "Profile-encoding reconstruction for multiple-acquisition balanced steady-state free precession imaging”. Magn. Reson. Med. 78 (2017), pp. 1316-1329. Dor:10.1002/mrm.26507 (cit. on p. 75).

[89] B. Bilgic, T. Witzel, H. Bhat, L. L. Wald, and K. Setsompop. "Joint Reconstruction of Phase-Cycled Balanced SSFP with Constrained Parallel Imaging”. Proc. Intl. Soc. Mag. Reson. Med. Vol. 25. Honolulu, 2017, p. 0441. U R L: http://indexsmart mirasmart.com/ISMRM2017/PDFfiles/0441.html (cit. on p. 75). 
[9o] D. L. Foxall. "Frequency-modulated steady-state free precession imaging". Magn. Reson. Med. 48 (2002), pp. 502-508. D o I: 10.1002/mrm.10225(cit. on pp.75 76, 81).

[91] T. Benkert, P. Ehses, M. Blaimer, P. M. Jakob, and F. A. Breuer. "Dynamically phasecycled radial balanced SSFP imaging for efficient banding removal". Magn. Reson. Med. 73 (2015), pp. 182-194. D O I: $10.1002 / \mathrm{mrm} .25113$ (cit. on p. 75 .

[92] A. Slawig, T. Wech, V. Ratz, J. Tran-Gia, H. Neubauer, T. Bley, and H. Köstler. "Multifrequency reconstruction for frequency-modulated bSSFP". Magn. Reson. Med. (2017). D I: $10.1002 / \mathrm{mrm} .26630$ (cit. on p. 75 ).

[93] V. Roeloffs, S. Rosenzweig, H. C. M. Holme, M. Uecker, and J. Frahm. "Frequencymodulated SSFP with radial sampling and subspace reconstruction: A time-efficient alternative to phase-cycled bSFFP”. Magn. Reson. Med.(2018). D o I:10.1002/mrm 27505 (cit. on p. 75).

[94] M. Schloegl, M. Holler, A. Schwarzl, K. Bredies, and R. Stollberger. "Infimal convolution of total generalized variation functionals for dynamic MRI". Magn. Reson. Med. 78 (2018), pp. 142-155. D O I: $10.1002 / \mathrm{mrm} .26352$ (cit. on p. 84 ).

[95] F. Ong. "Low Dimensional Methods for High Dimensional Magnetic Resonance Imaging”. PhD thesis. University of California, Berkeley, 2018. U R L: http://www2 eecs.berkeley.edu/Pubs/TechRpts/2018/EECS-2018-181.html (cit. on p. 84). 


\section{Danksagung}

Hier möchte ich zuerst Prof. Dr. Martin Uecker für die herausragende Betreuung danken: $\mathrm{Zu}$ jeder der Arbeit habe ich bei dir Rat und Ermutigung und Enthusiasmus gefunden. Vielen Dank für die tolle Zeit!

Weiterhin möchte ich mich bei Prof. Dr. Stefan Luther und PD Dr. Peter Dechent bedanken, die mich im Betreuungsausschuss mit Rat und mit ihrer Erfahrung unterstützt haben.

Diese Arbeit ist auch ein Resultat der kooperative Kultur in der Arbeitsgruppe: Ich konnte hier immer hilfsbereite und diskussionsfreudige Kollegen finden, wodurch viele Probleme schon „im Keim ersticken“ werden konnten. Daher möchte ich mich bei allen meinen Kollegen, und besonders bei Dr. Robin Wilke, Nick Scholand, Sebastian Rosenzweig, Dr. Xiaoqing Wang und Dr. Zhengguo Tan bedanken. Ein besonderer Dank gilt hier Sebastian Rosenzweig für die schöne und produktive Zeit im gemeinsamen Büro!

Trotz des letztendlich sehr engen Zeitplans hat Nick Scholand diverse Versionen dieser Arbeit Korrektur gelesen. Danke dafür!

Danke dir, Christina Bömer, dass ich den ganz normalen Wahnsinn des Promovierens mit dir teilen konnte.

Und zuletzt möchte ich Jess danken, ohne die ich die letzten Monate der Arbeit nicht überstanden hätte. Danke.

Ich möchte Prof. Dr. Thomas Pruschke für das $\mathrm{L}_{\mathrm{A}} \mathrm{T} \mathrm{E}-\mathrm{Template}$ danken, welches ich für diese Arbeit angepasst habe. 


\section{H. Christian M. Holme}

\section{Personal Information}

Born on 5th August 1990 in Soest, Germany

\section{University Education}

\section{PhD Program}

since 2016 at the University Medical Center Göttingen, in the International Max Planck Research School "Physics of Biological and Complex Systems" of the Göttingen Graduate School for Neurosciences, Biophysics, and Molecular Biosciences (GGNB), Working Title: Advanced Methods for Cardiovascular MRI, Supervisor: Prof. Dr. Martin Uecker.

\section{Master's Degree Progam in Physics}

2012 - 2016 at the Georg-August-Universität Göttingen, focus on "biophysics and physics of complex systems".

04/2016 Master's Degree, with distinction, Grade: 1.1.

02/2016 Master's Thesis, Biomedzinische NMR Forschungs GmbH (non-profit), at the Max Planck Institute for Biophysical Chemistry, Title: Preparatory data analysis for the reconstruction of real-time MRI data.

Supervisor: Prof. Dr. Jens Frahm, Grade: 1.0

08/2013 - 03/2014 Erasmus student exchange, at Stockholm University.

\section{Bachelor's Degree in Physics}

$2009-2012$ at the Georg-August-Universität Göttingen.

10/2012 Bachelor's Degree, focus on "biophysics and physics of complex systems", Grade: 1.7.

10/2012 Bachelor's Thesis, Institute for X-Ray Physics, Georg-August-Universität Göttingen, Title: Characterisation of EUV- and soft X-ray beams for coherent imaging. Supervisors: Dr. Dong-Du Mai and Prof. Dr. Tim Salditt, Grade: 1.3

06/2012 Experiments at BESSY II at the Helmholtz-Zentrum Berlin

05/2012 Experiments at the ESRF in Grenoble, France

\section{School Education}

2002 - 2009 Abitur, Humboldt-Gymnasium, Bad Pyrmont, Germany. Advanced Courses: Physics, Mathematics and English; Grade: 1.1

2006 - 2007 Language Exchange, Rainier Christian High School, Auburn, WA, USA.

\section{Languages}

German native language

English fluent (speaking, reading, writing) 


\section{Publications}

2019 Holme, H. C. M., Rosenzweig, S., Ong, F., Wilke, R. N., Lustig, M., Uecker, M., 'ENLIVE: An Efficient Nonlinear Method for Calibrationless and Robust Parallel Imaging'. Sci. Rep. 9.1, p. 3034. DoI: $10.1038 / \mathrm{s} 41598-019-39888-7$.

Rosenzweig, S., Holme, H. C. M., Uecker, M., 'Simple auto-calibrated gradient delay estimation from few spokes using Radial Intersections (RING)'. Magn. Reson. Med. 81.3, pp. 1898-1906. DOI: $10.1002 / \mathrm{mrm} .27506$.

2018 Roeloffs, V., Rosenzweig, S., Holme, H. C. M., Uecker, M., Frahm, J., 'Frequencymodulated SSFP with radial sampling and subspace reconstruction: A time-efficient alternative to phase-cycled bSFFP'. Magn. Reson. Med.81.3, pp. 1566-1579. Dor: 10.1002/ mrm. 27505.

Rosenzweig, S., Holme, H. C. M., Wilke, R. N., Voit, D., Frahm, J., Uecker, M., 'Simultaneous Multi-Slice MRI Using Cartesian and Radial FLASH and Regularized Nonlinear Inversion: SMS-NLINV'. Magn. Reson. Med. 79.4, pp. 2057-2066. D o I: 10.1002/mrm. 26878.

2016 Holme, H. C. M., Frahm, J., 'Sinogram-based coil selection for streak artifact reduction in undersampled radial real-time magnetic resonance imaging'. Quantitative Imaging in Medicine and Surgery 6.5, pp. 552-556. D o I: 10.21037/qims.2016.10.02. 